A Plasma Aerocapture and Entry System for Manned Missions and Planetary Deep Space Orbiters

\title{
A Plasma Aerocapture and Entry System for Manned Missions and Planetary Deep Space Orbiters
}

\author{
Phase I Final Report \\ PREPARED BY: \\ David Kirtley \\ MSNW LLC. \\ $8551154^{\text {th }}$ Ave NE \\ Redmond, WA 98052 \\ Phone: (425) 8678900 \\ Email: dkirtley@msnwllc.com \\ Contract Number: NNX12AR12G \\ Report Number: Final Report
}

DISTRIBUTION STATEMENT A - Public Release; Distribution is Unlimited. 
A Plasma Aerocapture and Entry System for Manned Missions and Planetary Deep Space Orbiters

MSNW LLC

\section{Table of Contents}

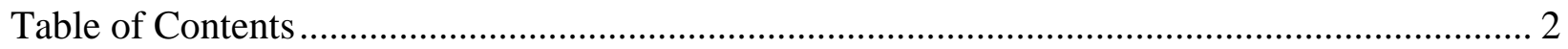

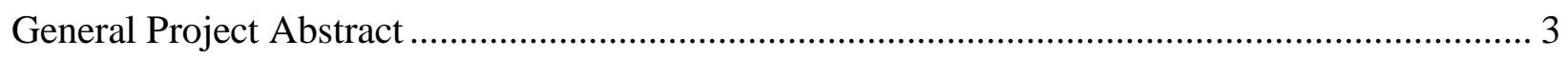

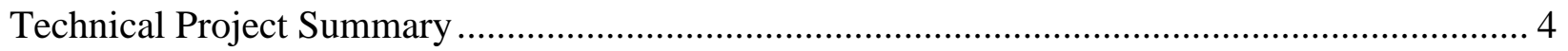

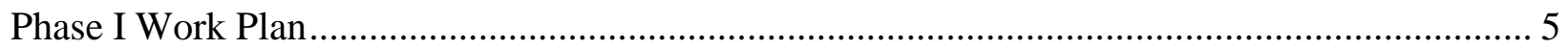

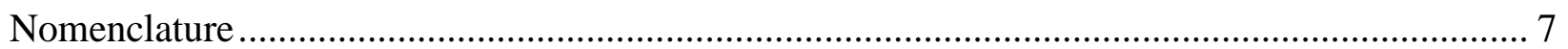

Background: Magnetoshell Physical Description ............................................................. 9

Basic parameter scaling of a Magnetoshell for Aerobraking ............................................ 9

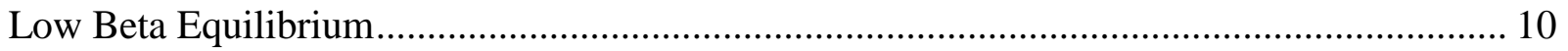

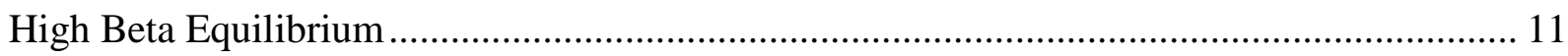

Direct Comparison With Previous Magnetic Sail Concepts ................................................ 13

Analytical Model of the Magnetoshell............................................................................. 14

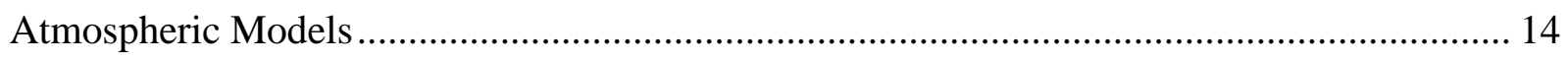

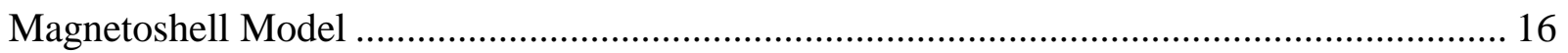

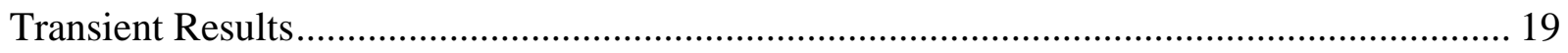

Related Modeling Effort - Sel HiFi............................................................................. 24

Laboratory Demonstration of an Argon Magnetoshell and Drag ........................................... 28

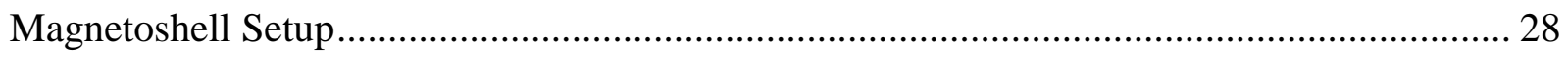

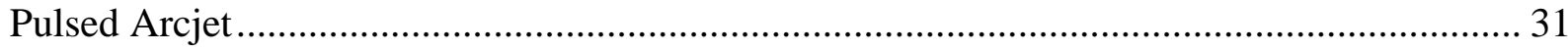

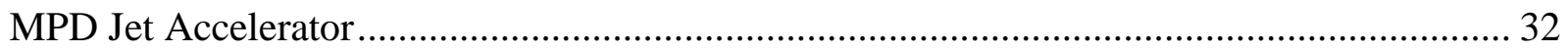

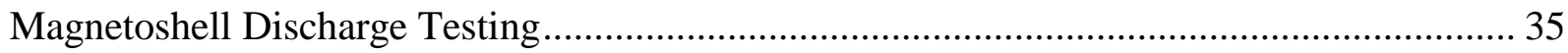

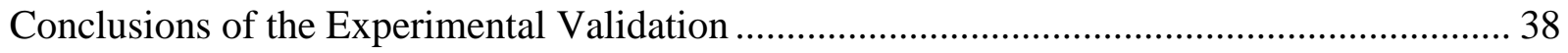

Orbital Modeling and General Mission Benefits ................................................................ 40

Mission Benefits and Architecture Consequences............................................................... 40

Neptune Orbiter Mission............................................................................................. 42

Magnetoshell Optimization and Design for Neptune Mission.............................................. 44

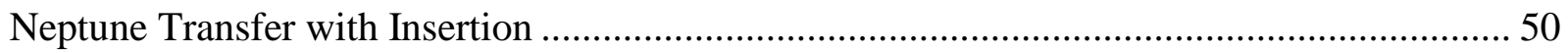

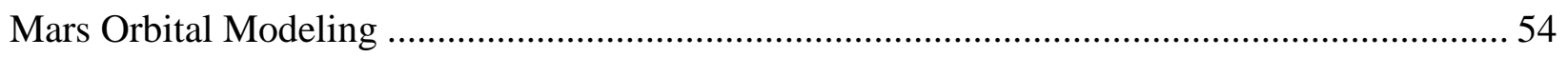

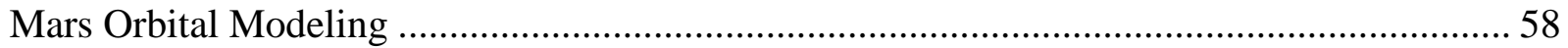

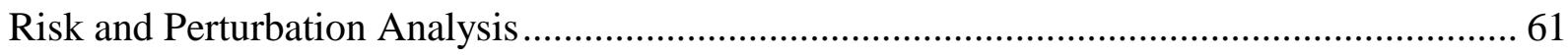

Conclusions from the Mission Design Study ................................................................. 63

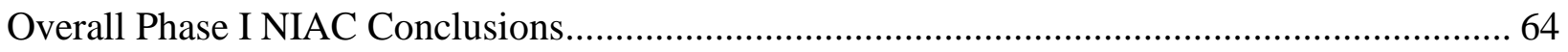

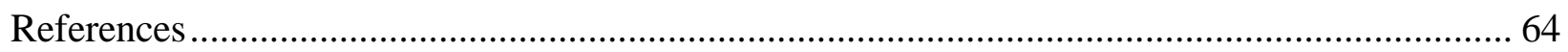


A Plasma Aerocapture and Entry System for Manned Missions and Planetary Deep Space Orbiters

MSNW LLC

\section{General Project Abstract}

A plasma Magnetoshell can enable a wealth of large scale inner planetary missions and deep space planetary orbiters. Aerocapture and aerobraking use aerodynamic drag in a planetary atmosphere in order to decelerate and shed velocity from a planetary transfer orbit. Aerobraking systems have been shown to dramatically reduce the mass and cost of interplanetary orbiters. Aerocapture systems use a high temperature ceramic Aeroshell and thermal protection system (TPS) to extend those benefits to allow not only orbit lowering, but injection orbit capture as well. Mission studies have shown the Aerocapture is a dramatically enabling technology for interplanetary science and manned missions and critical for the future of NASA space travel. The Magnetoshell aerocapture deploys a magnetic field filled with a magnetized plasma. It is interaction of the atmosphere with this plasma that supplies a significant impediment to atmospheric flow past the spacecraft, and thereby producing the desired drag for braking. The plasma based Magnetoshell being developed in this program holds the potential to perform the desired braking with significantly increased drag and control while reducing mass. Most importantly, as the drag can be varied dynamically this technology significantly lowers the risk involved with aerocapture thereby making interplanetary aerocapture possible without detailed a prior knowledge of a planet's atmosphere.

In Phase I a full system was designed for Neptune and Mars missions. This analysis showed that a $200 \mathrm{~kg}, 9 \mathrm{~m}$ Magnetoshell provides Neptune aerocapture for a $21 \mathrm{~km} / \mathrm{s}$

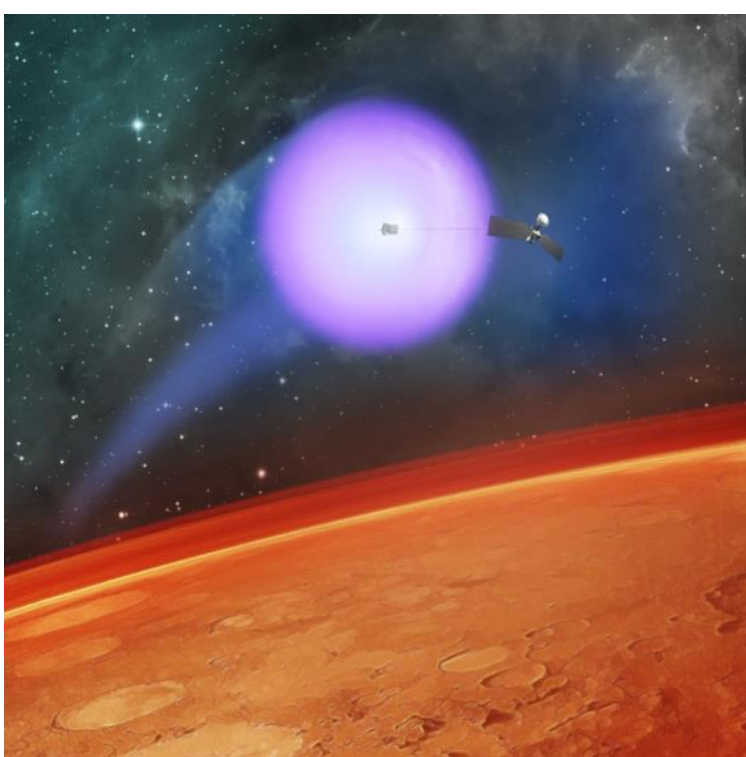

Figure 1. The Plasma Magnetoshell in a Mars aerobraking mission.

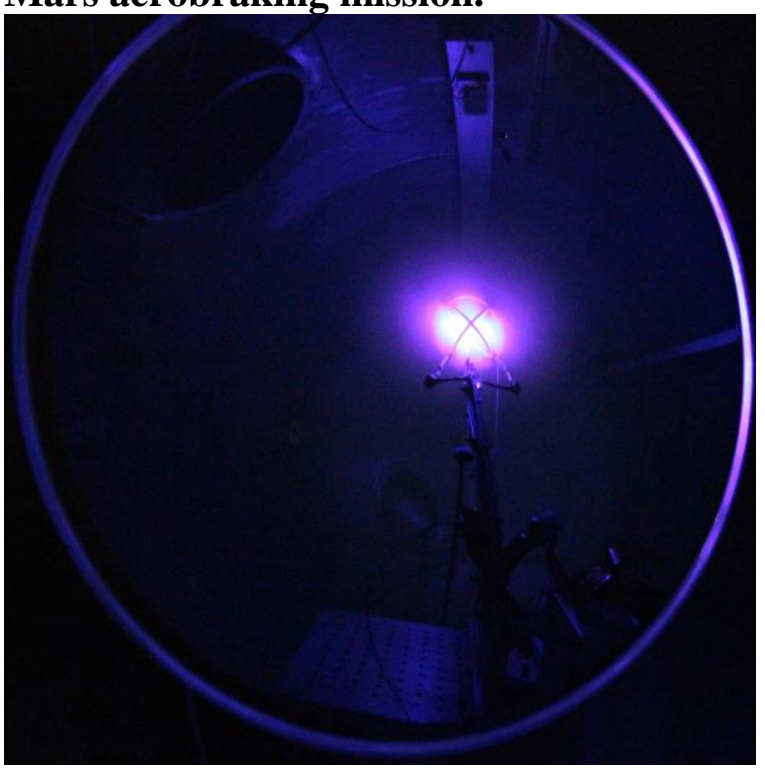

Figure 2. Magnetoshell operating with internal gas field and intercepting a partially ionized jet.

injection with a peak force of $150 \mathrm{~N}$. For a manned Martian aerocapture, a 21 meter Magnetoshell can be developed to provide aerocapture for a 60 metric ton payload. A transient analytic model was developed evolving the radial plasma parameters for a variety of plasma, neutral, and magnetic parameters. Finally, a stationary 2 meter argon Magnetoshell was fully demonstrated and a 1000:1 increase in aerodynamic drag was found. 
A Plasma Aerocapture and Entry System for Manned Missions and Planetary Deep Space Orbiters

MSNW LLC

\section{Technical Project Summary}

A rapid mission to Mars requires a large change in vehicle velocity upon arrival to establish a stable orbit. This demand is even greater for a Neptune science, requiring many kilometers per second of $\Delta \mathrm{V}$. It is clear from past mission studies that a manned Mars mission and deep space planetary orbiters require aerobraking and aerocapture which use aerodynamic drag forces to slow the spacecraft. Aerocapture would enable long term studies of the outer planets and moons that would not be possible with existing braking methodologies. While the ability to utilize these atmospheres to slow down and capture spacecraft would dramatically reduce the cost, launch mass, and travel time, currently planned approaches require significant additional spacecraft mass and risk as the spacecraft must descend deep into the planetary atmosphere in order to produce significant drag on a relatively small aeroshell. The plasma based Magnetoshell being developed in this program holds the potential to perform the desired braking with significantly increased drag and control while dramatically reducing mass. Most importantly, this technology significantly lowers the risk involved with aerocapture thereby making manned planetary missions possible. The fundamental physics of the Magnetoshell is based on demonstrated experimental results. Successful implementation will dramatically decrease radiation exposures, mission risk, launch cost, and launch mass.

Implementation of aerobraking by employing a solid deflector or aeroshell as a method for orbit insertion and circularization has been successfully demonstrated in the past, resulting in launch mass savings greater than $50 \%$. In order to reduce the effect of frictional heating and dynamic pressure on the typically fragile aeroshell, or worse solar panels, the braking must be distributed over many orbital passes at a high altitude in the less dense regions of the atmosphere. It can thus take several months for a meter-scale, $1000 \mathrm{~kg}$, aeroshell to execute the many elliptic orbital passes through the atmosphere to achieve the required $\Delta \mathrm{V}$. This rather slow method of braking not only reduces frictional heating and dynamic forces, but also avoids unpredictable dynamic behavior due to turbulence, as well as unknown and seasonally variable atmospheric composition and temperature which has led to dangerous, missioncritical events. For exploration class missions such as DRA 5.0 aerocapture and Thermal Protection Systems (TPS) are proposed for

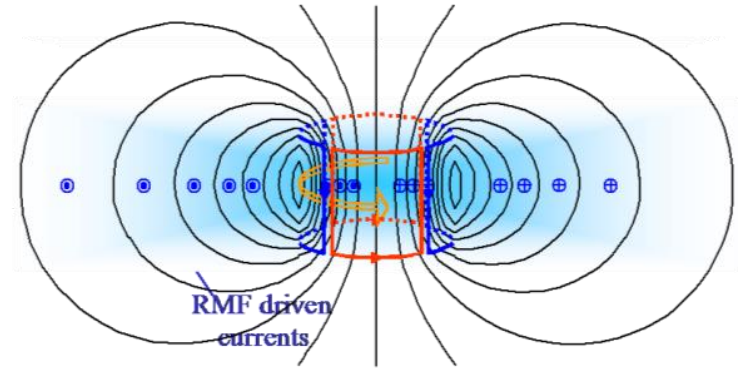

Figure 3. A Rotating Magnetic Field forms a magnetized plasma in a magnetic dipole. This plasma fills the dipole, providing, charge exchanging with incoming neutral flow, and dragging on the ambient background. breaking at Mars for cargo missions. Traditional aerocapture is considered too risky for manned missions. Even with the enormous mass savings that aerocapture allows, it still requires 80 tons of aeroshell and significantly increased launch mass and propellant. As will be shown, by using Magnetoshells for aerobraking, the DRA 5.0 mission will save 224 metric tons (MT) and greater than \$2 B in launch costs. Beyond the dramatic savings for existing mission architectures, a lowmass, risk-free aerocapture system would allow much more rapid missions to Mars and deep space orbiters by allowing direct, faster trajectories. As will be shown, the plasma Magnetoshell 
A Plasma Aerocapture and Entry System for Manned Missions and Planetary Deep Space Orbiters

MSNW LLC

Aerobraking, Aerocapture, and Entry System (AAES) not only reduces mass and cost while enabling significant new mission architectures, but also significantly reduces radiation exposures by decreasing trip times.

It is clear that aerobraking and aerocapture systems having low mass and complete stability control would enable both manned Mars and deep space missions. The Magnetoshell AAES deploys a magnetic field filled with a magnetized plasma. It is interaction of the atmosphere with this plasma that supplies a significant impediment to atmospheric flow past the spacecraft, and thereby producing the desired drag for braking. The advantages of such a system are many. Frictional heating would no longer be of concern as the energy dissipation required to slow the spacecraft would be deposited into the plasma ions helping to maintain the Magnetoshell's plasma content and at the same time shielding the spacecraft itself from frictional heating. With the aeroshell now being composed of massless magnetic field the transverse scale of the magnetic barrier can be as large as 100 meters while requiring no more than a gram of plasma. The Magnetoshell is also a highly dynamic control surface that can be regulated through either the magnitude of the magnetic dipole or the rate of plasma insertion into the dipole field. With the ability to rapidly and precisely modify the drag in varying atmospheric conditions, much larger braking forces can now be contemplated at low risk, enabling very aggressive aerocapture maneuvers.

Magnetoshell Aerocapture is a low- $\beta$ dipole plasma configuration which would initially be populated with ambient atmospheric gases (oxygen and nitrogen for Earth). This plasma is formed with an electrodeless Rotating Magnetic Field (RMF), which has been shown in previous experiments to generate the required, fully ionized, high temperature $(10 \mathrm{eV})$ magnetized plasma. The primary drag-inducing interaction between the magnetically confined plasma ions and the incoming neutral atmospheric particles is that of charge exchange, which has the largest cross section. After a collision, the now magnetized atmospheric ion reacts its directional momentum onto the magnet via field line bending and stretching.

In Phase I a full system was designed for Neptune and Mars missions. This analysis showed that a $200 \mathrm{~kg}, 9 \mathrm{~m}$ Magnetoshell provides Neptune aerocapture for a $21 \mathrm{~km} / \mathrm{s}$ injection with a peak force of $150 \mathrm{~N}$. A 21 meter Magnetoshell can be developed to provide aerocapture for a 60 metric ton payload. A transient analytic model was developed evolving the radial plasma parameters for a variety of plasma, neutral, and magnetic parameters. Finally, a stationary 2 meter argon Magnetoshell was fully demonstrated and a 1000:1 increase in aerodynamic drag was found.

\section{Phase I Work Plan}

The primary goal of the Phase I effort was to bring a Magnetoshell AAES system from TRL 2 to TRL 3. Phase I did seek to determine the feasibility, system parameters, and perform a subscale demonstration of a Plasma Magnetoshell for Aerobraking and Aerocapture. This consisted of a large quantity of mission studies as well as a thorough system trade study to determine overall system mass, power requirements, and mission benefits. By leveraging a large amount of existing hardware, a subscale, $1 \mathrm{~kW}$ Magnetoshell was demonstrated in the laboratory. In Phase II, these missions will be extended with teamwork with NASA and TPS scientists and a cubesat compatible demonstration will be performed. At the conclusion to this program, a 
A Plasma Aerocapture and Entry System for Manned Missions and Planetary Deep Space Orbiters

\section{MSNW LLC}

lightweight, practical Magnetoshell will have been developed and demonstrated that will significantly expand the capability of both manned missions and deep space orbiters. MSNW's demonstrated commercialization expertise is key to further development of this concept and eventual space demonstration.

Step 1 - Mission definition, simulation, and payoff

(a) Examine missions, both manned and unmanned, for which Magnetoshell AAES is enabling

(b) Select missions for detailed analysis, ideally Mars Exploration and Jupiter Icy Moon Orbiters

(c) Design and optimize mission plan use COPERNICUS

(d) Determine code development cycle for SEL-HiFi 3D MHD code

Step 2 - Subscale Demonstration

Leverage existing DARPA-funded RMF thruster electronics to construct a $1 \mathrm{~kW}$ Magnetoshell Leverage existing AFRL-funded arcjet hardware to construct neutral flow simulator

(b) Demonstrate Magnetoshell formation and drag on the MSNW dielectric thrust stand

Step 3 - Vehicle and subsystem design

(a) Based on the chosen mission destination, determine required system mass and basic system scale

(b) Design Magnetoshell-vehicle interface and address interaction concerns

(c) Design system-level requirements for required gaseous propellant and power system

(d) Determine experimental critical function tests for proof of concept for Phase II 
A Plasma Aerocapture and Entry System for Manned Missions and Planetary Deep Space Orbiters

MSNW LLC

\section{Nomenclature}

\begin{tabular}{|c|c|c|}
\hline$A_{\mathrm{pms}}$ & $=$ & Effective area of plasma Magnetoshell for interaction with planetary atmosphere \\
\hline$\alpha_{\mathrm{b}}$ & $=$ & Specific mass of the battery $[\mathrm{kg} / \mathrm{kW}]$ \\
\hline$\alpha_{\mathrm{p}}$ & $=$ & Specific mass of the power generation system $[\mathrm{kg} / \mathrm{kW}]$ \\
\hline $\mathrm{B}_{\mathrm{RMF}}$ & $=$ & Magnitude of rotating magnetic field \\
\hline $\mathrm{B}$ & $=$ & Magnitude of steady axial magnetic field \\
\hline $\mathrm{B}_{0}$ & $=$ & Magnitude of dipole field at coil center \\
\hline Isp & $=$ & Specific Impulse \\
\hline$\delta$ & $=$ & Classical skin depth in a conductor \\
\hline $\mathrm{D}_{\perp}$ & $=$ & Particle diffusion coefficient $=\eta / \mu_{0}$ \\
\hline $\mathrm{D}_{\text {bohm }}$ & $=$ & Particle diffusion coefficient assuming Bohm scaling \\
\hline$\Delta \mathrm{t}_{\mathrm{b}}$ & $=$ & Magnetoshell capture time, 2-sigma approximation \\
\hline$\Delta \mathrm{t}_{\mathrm{c}}$ & $=$ & Coast time between atmosphere interaction \\
\hline$\phi$ & $=$ & Dipole magnetic flux \\
\hline$\psi$ & $=$ & Dipole flux function \\
\hline $\mathrm{F}_{\mathrm{d}}$ & $=$ & Drag force on spacecraft \\
\hline$\eta$ & $=$ & Plasma resistivity \\
\hline I & $=$ & Current in dipole winding \\
\hline $\mathrm{I}_{\mathrm{c}}$ & $=$ & Total dipole coil current $(=\mathrm{N} \cdot \mathrm{i})$ \\
\hline $\mathrm{kb}$ & $=$ & Boltzmann constant \\
\hline$\lambda_{\mathrm{sc}, \mathrm{CE}}$ & $=$ & Total mean free path for charge exchange and neutral-ion elastic scattering \\
\hline 1 & $=$ & Magnet axial length \\
\hline $\mathrm{L}_{0}$ & $=$ & Scale length \\
\hline $\mathrm{m}_{\mathrm{g}}$ & $=$ & Atomic/molecular mass of planetary gas particle \\
\hline $\mathrm{m}_{\mathrm{i}, \mathrm{e}}$ & $=$ & Atomic mass of a ion or electron \\
\hline$\mu_{0}$ & $=$ & Magnetic permeability in vacuum \\
\hline $\mathrm{n}_{\mathrm{g}, \mathrm{n}}$ & $=$ & Local planetary neutral density for a given gas species \\
\hline $\mathrm{n}_{\mathrm{e}, \mathrm{i}}$ & $=$ & Plasma electron, ion density \\
\hline $\mathrm{n}_{\text {cex }}$ & $=$ & Charge exchange ion density \\
\hline $\mathrm{n}_{\text {ion }}$ & $=$ & Ionization ion density \\
\hline $\mathrm{n}_{0}$ & $=$ & Plasma density near dipole coil \\
\hline $\mathrm{N}$ & $=$ & Number of turns on dipole coil \\
\hline $\mathrm{N}_{\mathrm{i}}$ & $=$ & Particle inventory of plasma magneto-shell \\
\hline $\mathrm{V}_{\mathrm{s}-\mathrm{s}}$ & $=$ & Equilibrium rate for ion-electron temperature $[1 / \mathrm{s}]$ \\
\hline $\mathrm{P}_{\mathrm{e}}$ & $=$ & Electrical power dissipated in the coil \\
\hline $\mathrm{P}_{\mathrm{RMF}}$ & $=$ & Power delivered into plasma by RMF antenna \\
\hline r & $=$ & Radial distance in cylindrical coordinates \\
\hline ri & $=$ & Ion gyroradius \\
\hline $\mathrm{R}_{0}$ & $=$ & Radius of the Rotating Magnetic Field Antenna and dipole coil \\
\hline $\mathrm{RMF}$ & $=$ & Rotating Magnetic Field \\
\hline$\rho_{\mathrm{i}}$ & $=$ & Ion gyroradius of a charge transfer ion in the plasma Magnetoshell \\
\hline
\end{tabular}


A Plasma Aerocapture and Entry System for Manned Missions and Planetary Deep Space Orbiters

\section{MSNW LLC}

$\rho_{\mathrm{m}} \quad=\quad$ Density of the coil material

$\rho_{\mathrm{c}} \quad=$ Resistivity of the coil material

$\sigma \quad=\quad$ Total ion-neutral cross section

$\sigma_{\mathrm{sc}} \quad=$ Coefficient of emissivity of the magnet

$\sigma_{\mathrm{CEX}}=$ Charge exchange cross section

$\sigma_{\text {ion }}=$ Ionization cross section

$\mathrm{t}=$ Magnet thickness

$\mathrm{T}_{\mathrm{e}, \mathrm{i}}=$ Electron, ion temperature in $\mathrm{eV}$

Tc $=$ Average temperature of the magnet

$\mathrm{Ti}=$ Ambient temperature

$\tau_{\mathrm{N}} \quad=$ Particle confinement time in plasma Magnetoshell

$\mathrm{v}_{\mathrm{s}} \quad=\quad$ Spacecraft velocity relative to planetary atmosphere

$\mathrm{V} 0=$ Average particle velocity 
A Plasma Aerocapture and Entry System for Manned Missions and Planetary Deep Space Orbiters

MSNW LLC

\section{Background: Magnetoshell Physical Description}

The Plasma Magnetoshell deploys a magnetic field filled with a relatively high pressure, magnetized plasma. It is interaction of the atmosphere with this plasma that supplies a significant impediment to atmospheric flow past the spacecraft, and thereby producing the desired drag for braking.

\section{Basic parameter scaling of a Magnetoshell for Aerobraking}

The primary drag-inducing interaction between the magnetically confined plasma ions of the Magnetoshell and the incoming neutral atmospheric particles (viewed from the frame of the spacecraft) is that of charge exchange, which has the largest cross section, as well as elastic scattering. After a charge exchange, the now magnetized atmospheric ion reacts its directional momentum (again in the frame of the spacecraft) onto the magnet via field line bending and stretching. Elastic ion collisions with atmospheric neutrals induce drag as well but in smaller magnitude.

There are several parametric conditions that must be satisfied for the Magnetoshell to provide the desired aerobraking. First, the location in the planetary atmosphere must be at

Two oscillating current Antennas phased at $90^{\circ}$ to create a RMF steady dipole field magnet

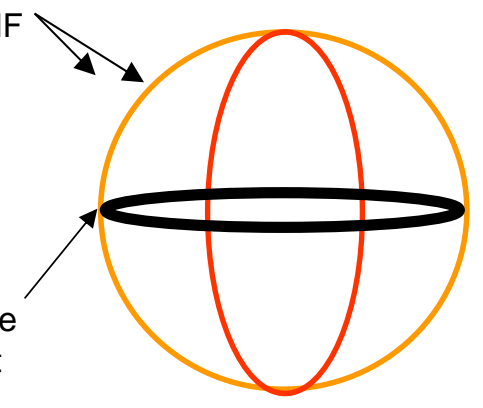

Figure 4. Coil set employed in generating a plasma Magnetoshell. The RMF antennas create a magnetic field that rotates about the axis of symmetry of the steady dipole magnet. an altitude where the local atmospheric drag on the spacecraft structure is itself small. For most atmospheres (including Earth's) this occurs at an ambient neutral number density of $\mathrm{n}_{\mathrm{g}} \sim 10^{19} \mathrm{~m}^{-3}$ or less. Secondly, the scale of the Magnetoshell at this density must be comparable to the mean free path for charge exchange. This sets the scale of the Magnetoshell at $10 \mathrm{~m}$ or larger assuming an ambient neutral density of $10^{18} \mathrm{~m}^{-3}$ or smaller. The third constraint relates to the minimum Magnetoshell magnetic field. To react the dynamic force onto the magnetic field, the charge exchanged atmospheric ions must stay magnetized long enough to be deflected by the local magnetic field (i.e. the charge exchange collision frequency must be less than the ion cyclotron frequency). Equivalently, the mean free path for charge exchange must be larger than the ion gyro-radius $\rho_{\mathrm{i}}$ of the charge-exchanged neutral. The newly created ions will have an effective temperature determined by the directed energy of the original neutral due to the motion of the spacecraft through the relatively motionless neutral gas.

The approximate braking force is found by considering the fraction of planetary neutrals trapped or scattered by the plasma magnetospheric ions. Because kinetic energy is conserved, resonant charge transfer acts as an elastic collision. At low energies $(<10 \mathrm{eV})$, the cross sections are large. Because the resonant charge transfer cross section is large, the particles are essentially undeflected in the center of mass (CM) system, leading after the charge transfer to an effective 
A Plasma Aerocapture and Entry System for Manned Missions and Planetary Deep Space Orbiters

MSNW LLC

scattering angle for the ion in the CM system of $180^{\circ}$ and a momentum transfer of $2 \mathrm{mg}_{\mathrm{g}} \mathrm{v}_{\mathrm{s}}$ for every collision, where $\mathrm{m}_{\mathrm{g}}$ is the mass of the charge exchange particle and $\mathrm{v}_{\mathrm{s}}$ is the spacecraft velocity. The drag force on the spacecraft is thus:

$$
\mathrm{F}_{\mathrm{d}}=2 \mathrm{~m}_{\mathrm{g}} \mathrm{n}_{\mathrm{i}} \mathrm{v}_{\mathrm{s}}^{2} \mathrm{~A}_{\mathrm{pm}}
$$

where $A_{p m}$ is the effective cross sectional area of the plasma shell. There is a minimum scale to assure that a passing neutral experiences at least one charge exchange or ion collision inside the Magnetoshell. The ion means free path is given by $\lambda_{\mathrm{sc}}=1 /\left(\mathrm{n}_{\mathrm{g}} \sigma_{\mathrm{sc}}\right)$ where $\sigma_{\mathrm{sc}}$ it the total ionneutral scattering cross section for both elastic and charge transfer collisions. For low energy collisions the scattering cross section $\sigma_{\mathrm{sc}} \sim 10^{-18} \mathrm{~m}^{2}$.

\section{Low Beta Equilibrium}

$\beta$ is the ratio of plasma pressure to magnetic pressure and is, in general, an indicator of the energy stored within a plasma. Plasma-filled dipoles can be either a low or high $\beta$ dipole, thus, both will now be discussed. In the low $\beta$ case, the plasma does not perturb the magnetic field significantly and the magnetic field radial profiles are the standard vacuum case. The energy and diamagnetic current in the plasma is low and cross-field diffusion is controlled by standard diffusion and lifetime scaling relationships. In a high $\beta$ dipole a large plasma current is generated. This plasma current produces a magnetic field which acts to add to the existing vacuum magnetic field, expanding the radial magnetic field profile. This allows for a slower falloff and a decrease in radial plasma diffusion. For a mission the high $\beta$ dipole would require a smaller, lower power magnet to provide the initial magnetic field as the plasma would expand the dipole field significantly. However, in practice to sustain this plasma current and lost (diffusing) magnetic flux requires significant power addition to the plasma. In the course of this study it was found that a High $\beta$ dipole is not necessary, and a low $\beta$ dipole is completely sufficient for aerobraking and aerocapture and that the energy required to sustain the plasma currents are larger than the corresponding magnetic power. For interstellar or solar-wind based missions it would be necessary to expand the dipole's effective radius of capture much larger than what would be possible in a low $\beta$ dipole. For this program, focusing on interplanetary aerocapture, a low $\beta$ dipole will be used.

The plasma does not generate a magnetic field and therefore does not affect the scaling of the dipole. A standard dipole magnetic field scaling of $1 / \mathrm{R}^{3}$ will be used. The confinement time and radius are as above and set by the same density and mean free path considerations. In order for a charge exchange to happen, the mean free path for a collision must be smaller than the dipole size and therefore the neutral density. The ion gyroradius must be less than the scale of the dipole in order for the ion to be confined and transfer momentum during a single orbit. If the collisional mean free path is much smaller than the gyroradius, the ions do not stay confined on the time scale of interest. Therefore as an ion gyroradius is independent of the collisionality, any required

similarly be described as simply the radial diffusion time of a particle across the radius of the dipole. Finally, for a low $\beta$ dipole, no energy is required to sustain the plasma except to replace lost particles; however, emitting an energy is required to sustain the dipole magnetic field. In the system analysis study, it'll be shown that this well within the limits of traditional copper magnet 
A Plasma Aerocapture and Entry System for Manned Missions and Planetary Deep Space Orbiters

MSNW LLC

technology. It is important that no super conducting or low TRL components are to required sustain the dipole magnetic field.

\section{High Beta Equilibrium}

For completeness, a high $\beta$ dipole will now be described. The RMF technology that was previously mentioned can be utilized to generate the high $\beta$ plasma. This can also be done with virtually any gas or gas mixtures to match the particular atmosphere of interest. The dipole magnetic field would be generated by a simple electromagnet.

The a high $\beta$ Magnetoshell consists of a pair of polyphase coils that produce a rotating magnetic field (RMF) that drives the necessary currents in the plasma to inflate and maintain the large-scale magnetic structure. Details of the physics of this process can be found elsewhere [3]. The current in the steady dipole is in the opposite direction to that produced in the plasma by the rotating magnetic field. The plasma currents thus act like a diamagnetic current creating a high $\beta$ plasma dipole equilibrium.

The large scale currents needed for a large-scale Magnetoshell are generated by the entrainment of the plasma electrons in a rotating field created by two pair of loop antennae (see Figure 3). With the antennae separated by 90 degrees in azimuth, and with their respective currents separated in phase by 90 degrees, a steady magnetic field rotating in the equatorial plane is produced. Analogous to the induction motor, this is the same as the field that is produced in a two pole motor stator winding.

Consider the case now where the metal rotor is replaced with a plasma rotor. With essentially zero inertial mass, the electrons quickly come into co-rotation with the RMF. The rigid rotation of the electrons is retarded slightly by collisions with the much more massive background ions which, due to their large inertia are unable to respond to the rapidly rotating field and remain relatively motionless. In this manner a large azimuthal $(\theta)$ current is driven in the plasma. The magnetic field generated by the rotor currents couple it inextricably with the stator fields. Like any AC motor, a force applied to the rotor is reacted back on to the stator through these fields without any physical contact between the two. The same momentum transfer occurs with the plasma rotor as well. The eventual "load" on the plasma rotor will be the drag of the neutrals as they are exchanged or scattered off the plasma magnet ions. This directional force is countered by the deflection of the dipole magnetic fields which, through the modification of the magnet currents, is reacted back onto the steady dipole magnetic coil (the stator) attached to the spacecraft.

With no external field to oppose the Lorentz self-force acting on the plasma azimuthal currents, the current ring expands. The only confining force is that of the curvature forces from the steady dipole field created by the RMF driven currents. This force is sufficient to keep the plasma in a high $\beta$ equilibrium as it expands (the driven current is equivalent to a plasma diamagnetic current in equilibrium). Since the gyroradius of a planetary neutral/ion will be much larger than the initially meter size expanding dipole, little distortion of the dipole is expected until the Magnetoshell has expanded enough to have significant interaction with the background atmosphere. In addition to expanding the dipole, the driven currents will change the equilibrium field shape significantly from the vacuum field.

Realistic equilibria for the magnetic dipole with large diamagnetic currents have been found 
A Plasma Aerocapture and Entry System for Manned Missions and Planetary Deep Space Orbiters

\section{MSNW LLC}

and analyzed [2]. It was found that plasma pressure profiles $\mathrm{P}(\psi)$ that fall off at least as slowly as the adiabatic rate $\left(\sim \psi^{20 / 3}\right)$, where $\psi$ is the flux surface coordinate, were stable to both interchange and ballooning instabilities [3]. The modification to the dipole flux function $\psi$ $\left(\sin (\theta)^{2} / \mathrm{r}\right.$ in vacuum) at high $\beta$ can be expressed as:

$$
\psi=\psi_{0} \mathrm{R}_{0}^{\alpha} \frac{\sin (\theta)}{\mathrm{r}^{\alpha}}, \quad \alpha=\beta_{0}^{-1 / 2}
$$

where the zero subscript refers to a reference surface near the RMF antenna. In the limit of $\beta \rightarrow$ $\infty$ (the plasma pressure is equal to the external field pressure), the dipole field $\mathrm{B}(\mathrm{r}) \sim \mathrm{B}_{0} \mathrm{R}_{0}{ }^{2} / \mathrm{r}^{2}$. With $\beta>1$, the dipole field drop-off will at best be no slower than $1 / \mathrm{r}^{2}$. This can be seen from flux conservation. It is clear that the high $\beta$ plasma will expand the flux over a much larger region (with equatorial area $\mathrm{A}_{\mathrm{pm}}$ ).

Once the expansion is complete, confining the plasma in a large scale Magnetoshell looks to be relatively undemanding. The simplest and most desirable method to achieve the desired density falloff is to simply reduce the particle flow from the source. If the confinement is adequate, or the entrainment of the planetary neutrals through ionization by electrons is sufficient to match losses, source plasma fueling may be completely turned off. If too great a density buildup should occur, it would be suppressed by the nature of the current drive process itself. As the density increases, the driven current increases. This in turn expands the dipole magnetic field, and leads to a further expansion of the Magnetoshell. The expansion drops the density, which reduces the current, and a new equilibrium is achieved. The expansion of the dipole is now only limited by the Ohmic power needed to maintain the structure against resistive dissipation. To make a simple estimation of what this power would be, consider the case of an azimuthally uniform magnetic field where the $1 / \mathrm{r}^{2}$ dependence is found in all directions. The RMF power, $\mathrm{P}_{\mathrm{RMF}}$, needed to sustain the plasma Magnetoshell in this configuration is given by:

$$
\mathrm{P}_{\mathrm{RMF}}=\int \eta \mathrm{j}_{\theta}^{2} \cdot \mathrm{dVol}
$$

where $j_{\theta}$ is the azimuthal current density driven by the RMF. Flux conservation demands that $\mathrm{B}(\mathrm{r}) \sim \mathrm{B}_{0} \mathrm{R}_{0}{ }^{2} / \mathrm{r}^{2}$, so that one has for $\mathrm{j}_{\theta}$ :

$$
\mathrm{j}_{\theta}=\frac{1}{\mu_{0}} \frac{\mathrm{dB}}{\mathrm{dr}}=\frac{-2 \mathrm{~B}_{0}}{\mu_{0}} \frac{\mathrm{R}_{0}^{2}}{\mathrm{r}^{3}}
$$

where $\mathrm{B}_{0}$ is the magnitude of the magnetic field at the center of the dipole coil. Substituting Eq. (3) into Eq. (4) and integrating one has:

$$
P_{\text {RMF }}=\frac{4 \eta}{\mu_{0}^{2}} B_{0}^{2} R_{0}^{4} \int_{R_{0}}^{r>R_{0}} \frac{4 \pi}{r^{4}} d r=\frac{16 \pi \eta}{3 \mu_{0}^{2}} B_{0}^{2} R_{0}
$$


A Plasma Aerocapture and Entry System for Manned Missions and Planetary Deep Space Orbiters

MSNW LLC

\section{Direct Comparison With Previous Magnetic Sail Concepts}

It must be emphasized that although there are similarities, this is not the interaction envisioned in concepts for obtaining magnetospheric plasma propulsion from the solar wind $[1,3,4]$. In these concepts the primary interaction is between an external plasma "wind" and a magnetospheric object. This supersonic plasma exerts a direct compressional effect on the magnetosphere through electromagnetic forces and the interaction is quite complex. While drag could be obtained from the planetary ionosphere by the interaction of the ambient planetary plasma and the Magnetoshell, the effect would be significantly smaller as the peak ion density in ionospheres is typically orders of magnitude smaller than the neutral density found at the lower altitudes considered here. In addition, the ionospheres of Mars and other objects of interest are not well understood, and can exhibit large changes in intensity on unpredictable timescales. Since the Magnetoshell-atmospheric interface is governed by small-scale, energy conserving particle interactions that do not directly affect the plasma dipole equilibrium, the behaviour becomes much more predictable and controllable.

The other primary difference is plasma beta. In both the mini-magnetospheric plasma propulsion (M2P2, winglee, slough) and the Plasma MagnetoSphere (slough) had large beta in which the effective falloff and dipole radius is much larger than the vacuum condition. In the M2P2 the magnetic field is sustained entirely by the incoming solar flow [3], by an unclear mechanism. The PMS magnetic flux is stained by direct drive of a rotating magnetic field [1] by inducing current directly as described above. In both of these designs the significant current drive and sustainment energy is required to inflate a magnetic dipole to many kilometer scales required for creating a significant force with a flow as weak as the solar wind. 
A Plasma Aerocapture and Entry System for Manned Missions and Planetary Deep Space Orbiters

\section{MSNW LLC}

\section{Analytical Model of the Magnetoshell}

A transient plasma model was developed. The development and results of this model will now be described.

\section{Atmospheric Models}

This 2-D model used empirical models for atomic collision cross section and atmospheric neutral gas densities for a variety of planets. Using empirical measurements averaged over the available probe data, neutral gas densities as a function of altitude are shown in Figure 5 through Figure 7. To give a sense of scale, neutral particle density of $1 \mathrm{E} 18 \mathrm{~m}^{-3}$ density for a variety of planets can be compared. In Figure 5; the earth reference altitude is 112 kilometers. On Mars, the reference altitude is 115 kilometers, while on Neptune the reference altitude is 700 kilometers above the nominal surface altitude. References for the empirical gas models are given in the

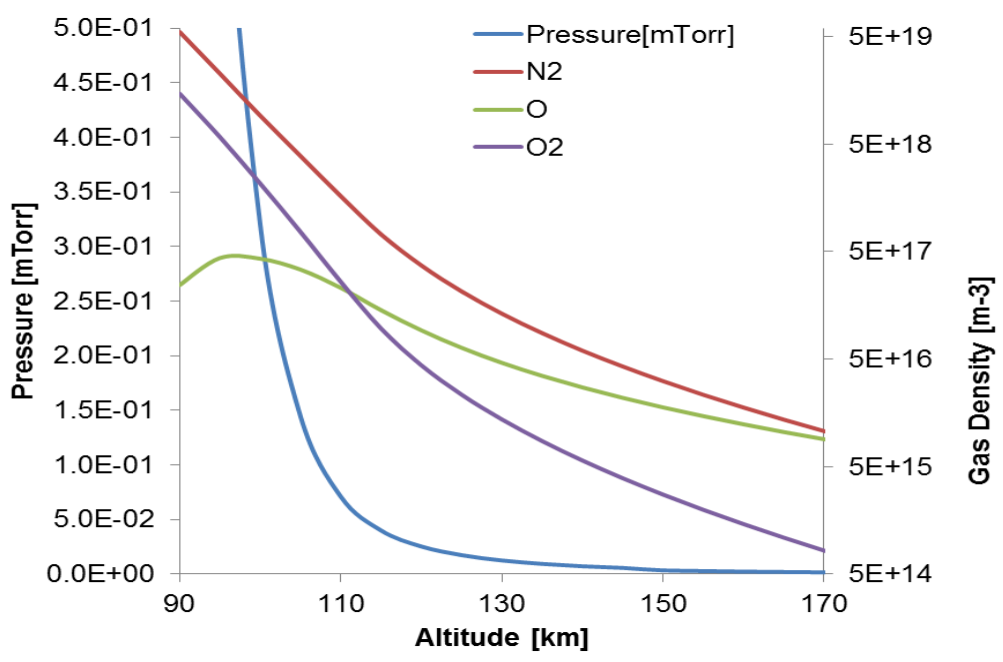

Figure 5. Earth neutral density by species and altitude. [5] 
A Plasma Aerocapture and Entry System for Manned Missions and Planetary Deep Space Orbiters

MSNW LLC

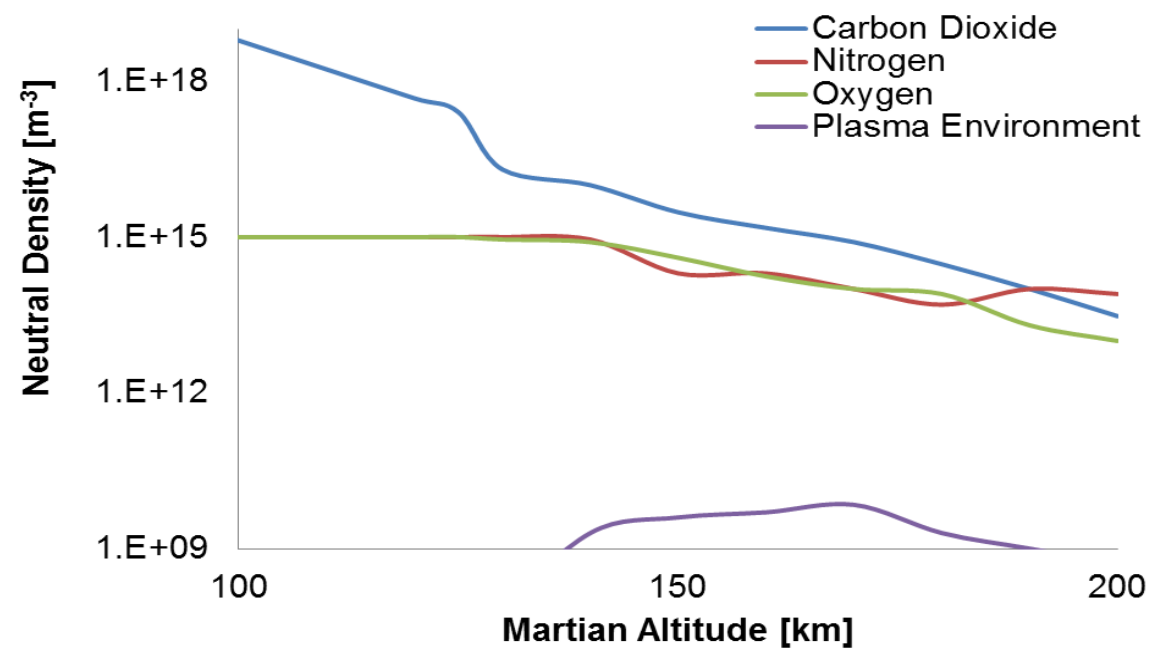

Figure 6. Martian neutral density by species and altitude. Shown also is average plasma density [6].

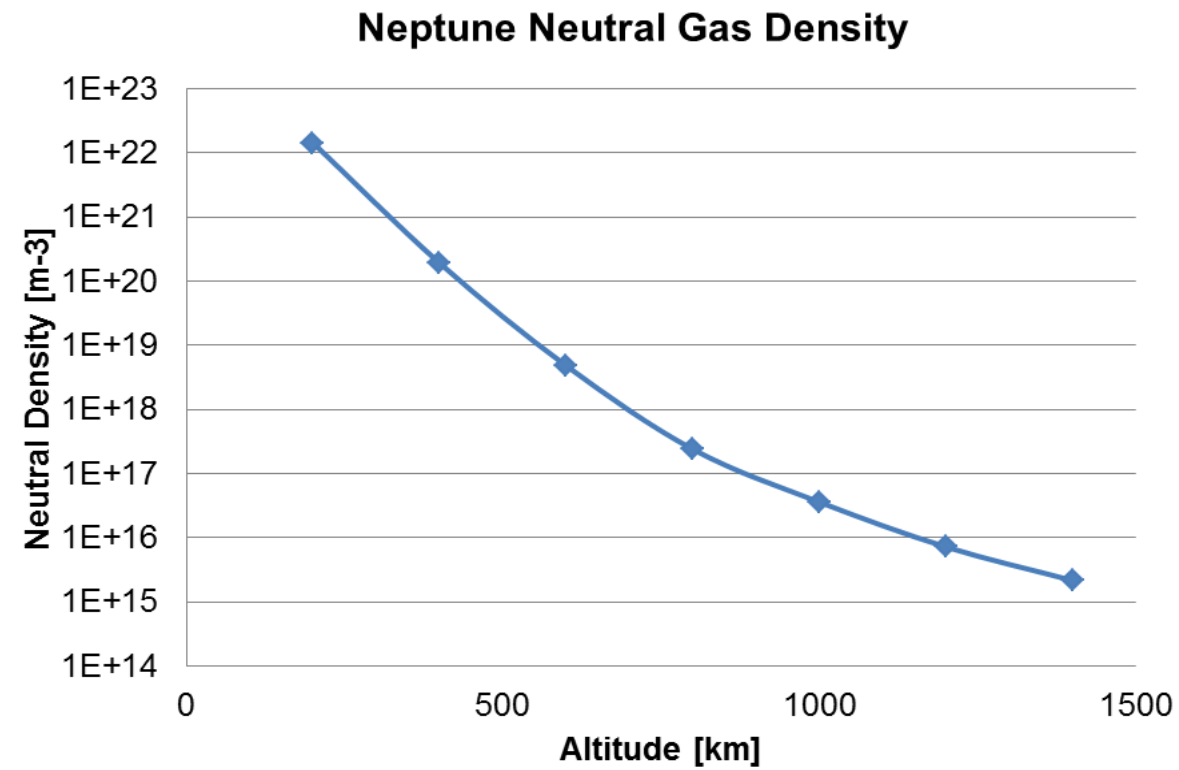

Figure 7. Neptune total neutral density. Average molecular weight is $2.5 \mathrm{amu}$ [7]. 


\section{A Plasma Aerocapture and Entry System for Manned Missions and Planetary Deep Space Orbiters}

MSNW LLC

\section{Magnetoshell Model}

In order to determine the equilibrium plasma and gas distribution of a Magnetoshell under a specific set of incoming flow characteristics, a 2-D transient model was developed. This model uses a cylindrical axisymmetric approximation. This cylindrical approximation has an aspect ratio of 3 and assumes uniform chord properties. This assumption is believed to be accurate for the typical mean free paths of interest in this experiment as only a few collisions will happen along a given cylindrical chord. Assuming a uniform chord property and uniform azimuthal plasma conditions, radial profiles of ion and electron densities and temperatures can be calculated. A uniform incoming neutral flow distribution is assumed with uniform velocity and

assumed. Previous reports have described low versus high-beta configurations, but the low-beta energy state is the lowest required plasma energy power, as well as the simplest plasma assumption. This low-beta configuration then had a cubic radial fall off of magnetic field and initial density distribution. Two more critical assumptions are made in a low-beta configuration; the plasma has little effect on the magnetic field, so a fixed magnetic field profile is assumed and finally, collisional cross sections use several empirical models to determine the inter-species interaction of neutrals, ions and electrons. The biggest assumption in this Phase I model is an atomic species profile, which uses approximate atomic species of a given atmosphere. For earth, an nitrogen atom is assumed. For Mars, an argon atom, while for Neptune, hydrogen is assumed. The interaction of molecular collisions will be investigated in Phase I. As the mean free path is large non-maxwellian, non-equilibrium plasma conditions are assumed, in which the plasma ions and electrons do not automatically relax to equilibrium states and equilibrium temperature distribution. Therefore, cross sections for charge exchange and ionization can be empirically determined for a given electron temperature [8-10]. 
A Plasma Aerocapture and Entry System for Manned Missions and Planetary Deep Space Orbiters

MSNW LLC

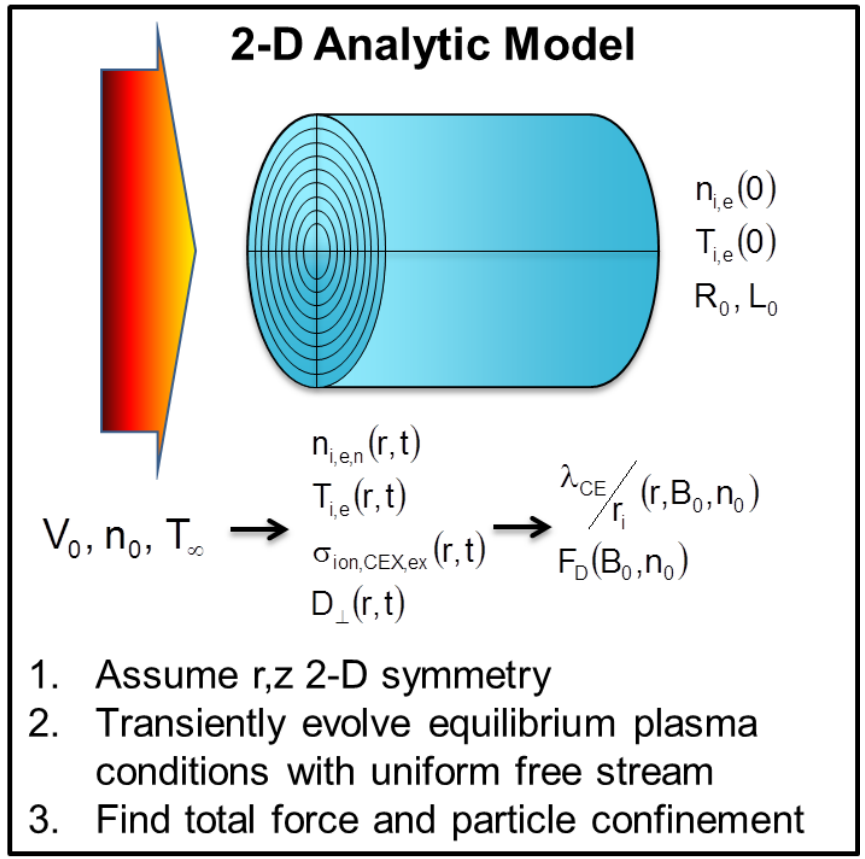

Figure 8. Summary of the 2D analytic model and key assumptions.

Using empirical cross section for charge exchange for a given atomic species and electron temperature, the charge exchange reaction rate equation can be calculated for each radial position averaged over a chord length. This requires neutral ion and electron densities, neutral velocities, and chord length scale. Using charge exchange, collision rates, the ion and neutral population does not change (as charge is simply exchanged, not created) but ion temperature and thermal energy equilibrates to the charge exchange temperature as a function of the charge exchange rate. Ionization is calculated as shown in Equation 6 and 7 from the temperature and species dependent cross section densities and mean free path. Ionization rates are used to recalculate ion population, as well as cool electrons. Electron temperature must decrease by the ion energies per ionization event. Plasma diffusion is calculated between layers as plasma diffuses radially outwards from the central core and the external boundary and ends. Two diffusion rates are used; classical (Spitzer) diffusion assumes high plasma conductivity and optimal plasma resistivity in Equation 9. This is simply diffusion due to the motion of a magnetized plasma particle. Bohm diffusion is applicable for a plasma population that undergoes many neutral collisions in which cross field diffusion of the plasma is dominated by collisional event rather than magnetized particle motion and is shown in Equation 8. These two diffusion rates bracket the minimum and maximum diffusion rates respectively and the average value is used to calculate the diffusion times and the equilibrium density. Finally, total equilibrium is calculated using a standard Fokker-Planck equilibrium rate. This rate determines the heating from ion to electron and electron to ion and again is shown in Equation 10. 
A Plasma Aerocapture and Entry System for Manned Missions and Planetary Deep Space Orbiters

MSNW LLC

$$
\begin{gathered}
\mathrm{n}_{\mathrm{CEX}}=\sigma_{\mathrm{CEX}}\left(\mathrm{T}_{\mathrm{e}}\right) \mathrm{n}_{\mathrm{n}} \mathrm{n}_{\mathrm{e}} \frac{\mathrm{V}_{\mathrm{o}}}{\mathrm{L}_{\mathrm{o}}} \Delta \mathrm{t} \\
\mathrm{n}_{\text {ion }}=\sigma_{\text {ion }}\left(\mathrm{T}_{\mathrm{e}}\right) \mathrm{n}_{\mathrm{n}} \mathrm{n}_{\mathrm{e}} \frac{\mathrm{V}_{\mathrm{o}}}{\mathrm{L}_{\mathrm{o}}} \Delta \mathrm{t} \\
\mathrm{D}_{\text {Bohm }} \propto \frac{\mathrm{k}_{\mathrm{B}} \mathrm{T}}{\mathrm{B}} \\
\mathrm{D}_{\perp} \propto \eta_{\perp} \frac{\mathrm{k}_{\mathrm{B}} \mathrm{T}}{\mathrm{B}^{2}} \propto \frac{\mathrm{m}_{\mathrm{i}} \mathrm{k}_{\mathrm{B}} \mathrm{T}^{1 / 2}}{\mathrm{~B}^{2}} \\
v_{\mathrm{s}-\mathrm{s}}=\frac{2 \mathrm{n}_{\mathrm{s}} \sigma_{\mathrm{T}} \ln \Lambda}{\pi^{1 / 2}}\left(\frac{\mathrm{m}_{\mathrm{e}}}{\mathrm{m}_{\mathrm{i}}}\right)\left(\frac{\mathrm{k}_{\mathrm{B}} \mathrm{T}_{\mathrm{e}}}{\mathrm{m}_{\mathrm{e}}}\right)^{-3 / 2}[1 / \mathrm{s}]
\end{gathered}
$$

Cubic density falloff is given in Equation 11 and is the initial starting distribution. The confinement requirements can then be determined. The mean free path for charge exchange is given in Equation 12 and is the average distance a particle will travel before undergoing a charge exchange collision. Equation 13 is the ion gyroradius and is the average distance an ion will travel is a magnetic field for a single oscillation or gyro-orbit. In order for charge exchange reactions to be dominant, the mean free path for CEX must be less than the size of the magnetic dipole region. Once a newly created charge exchange ion is magnetized, it must stay magnetized long enough to transfer its momentum to the structure. Therefore Equation 14 shows the relationships that must be maintained for a CEX collision to transfer momentum to the spacecraft.

$$
\begin{gathered}
\mathrm{n}(\mathrm{r}) \sim \mathrm{n}\left(\mathrm{R}_{0}\right)\left(\frac{\mathrm{R}_{0}}{\mathrm{r}}\right)^{3} \\
\lambda_{\mathrm{CE}}=\frac{1}{\mathrm{n}_{\mathrm{n} 0} \sigma_{\mathrm{CE}}} \\
r_{i}=\frac{m V_{0}}{e B} \\
\pi \mathrm{r}_{\mathrm{i}}<\lambda_{\mathrm{CE}} \leq \mathrm{L}_{0}
\end{gathered}
$$




\section{A Plasma Aerocapture and Entry System for Manned Missions and Planetary Deep Space Orbiters}

MSNW LLC

\section{Transient Results}

General trends can be seen. First given sufficient time, ion temperature will equilibrate with the average temperature of the incoming flow. This is the equivalent directed energy of an incoming neutral velocity. For typical aerobraking applications, incoming neutral energies 5 to $30 \mathrm{eV}$ are typical. Electrons equilibrate to a temperature somewhat lower, depending on the specific ionization rate. Ion density will equilibrate to a fixed value based off of the incoming temperature and diffusion rates for incoming flow energy is less than a typical ionization energy (12 to $20 \mathrm{eV}$ ) particles decay, particle density decays at the diffusion rate, while sustained by limited ionization as greater than the ionization energy significant increase in ionization occurs, resulting in an expansion of the Magnetoshell until a matching radial equilibrium condition is met in which ionization rate and diffusion rate are equal.

Radial profiles as shown in Figure 9 are also quite intuitive. Regardless of the initial density or temperature distribution, the radial profile quickly relaxes to a peaked profile, in which external diffusion is supported by the higher density core of the Magnetoshell. Temperature also relaxes to a peaked profile quite quickly.

Time scales and interest are somewhat longer than expected. Thermal equilibrium occurs in a few milliseconds at the core, while the radial profile may take many tens of milliseconds to fully evolve. Density distributions evolve over the time scales of the confinement time, which for these devices and typical operating conditions will be many minutes or longer. Figure 9 and Figure 10 show relevant time scale. 
A Plasma Aerocapture and Entry System for Manned Missions and Planetary Deep Space Orbiters

MSNW LLC

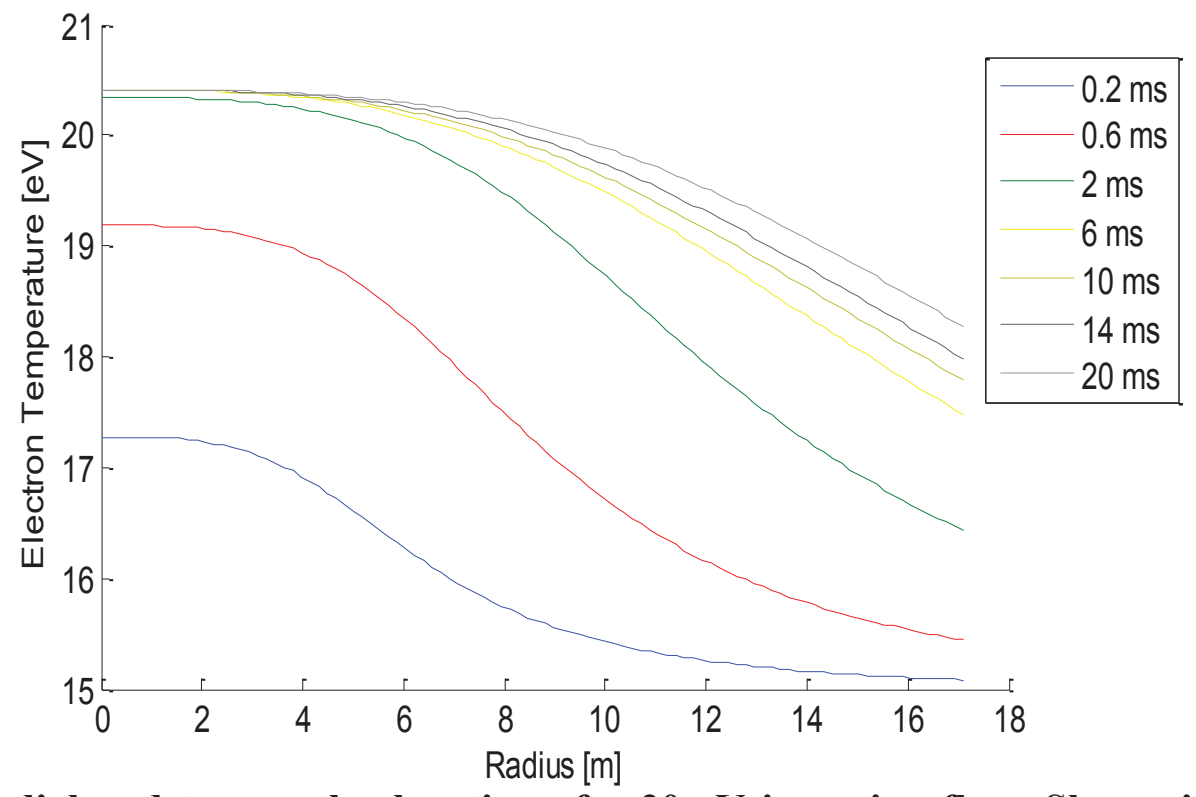

Figure 9. Radial and temporal relaxation of a $20 \mathrm{eV}$ incoming flow. Shown is Venus, 10 $\mathrm{km} / \mathrm{s}$ insertion.

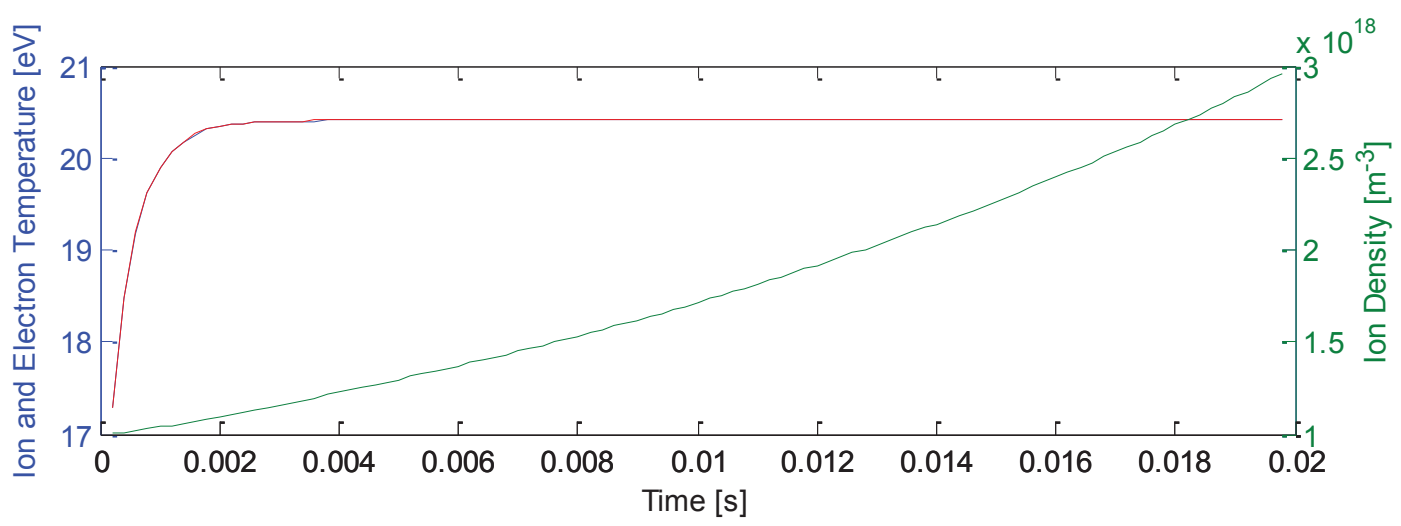

Figure 10. Ion and Electron Equilibration times are $\sim 1 \mathrm{~ms}$, while ion equilibration may take minutes. Shown is Venus, $10 \mathrm{~km} / \mathrm{s}$ insertion. 


\section{A Plasma Aerocapture and Entry System for Manned Missions and Planetary Deep Space Orbiters}

\section{MSNW LLC}

Using the empirical atmosphere model and equilibrium conditions of the Magnetoshell, the relative diameter, and drag magnitude can now be determined. As described in previous reports with a known plasma and neutral density and temperature, confinement can be determined. Two confinement relationships are critical and shown in Equations 11 to 14. First, the charge exchange mean free path is the effective distance for a charge exchange collision. That distance must be less than the effective radius or chord length of the system. Additionally, the magnetization ion gyroradius must be less than this critical distance and more than the charge exchange mean free path. This is required so that significant momentum can be converted from directed momentum to average equilibrium momentum. The equations are shown in Figure 8. Therefore, an affective diameter can be calculated, and using that affective diameter in a crosssectional area, a total drag can be determined. Figure 11 and Figure 12 show Martian results for a Magnetoshell. At $120 \mathrm{~km}$, there is a drag force of $1 \mathrm{kN}$ with an affective radius of $15 \mathrm{~m}$. In this case, all power and plasma parameters are provided by the incoming flow, and the only power is provided to maintain the magnetic field. Figure 13 and Figure 14 show Neptunian results. At $1000 \mathrm{~km}$ there is an affective drag force of $100 \mathrm{~N}$ with a radius of $17 \mathrm{~m}$, and all power, temperature, and plasma energy is sustained by the incoming flow. Clearly significant forces can be generated by this technique to brake in planetary atmospheres. 
A Plasma Aerocapture and Entry System for Manned Missions and Planetary Deep Space Orbiters

MSNW LLC

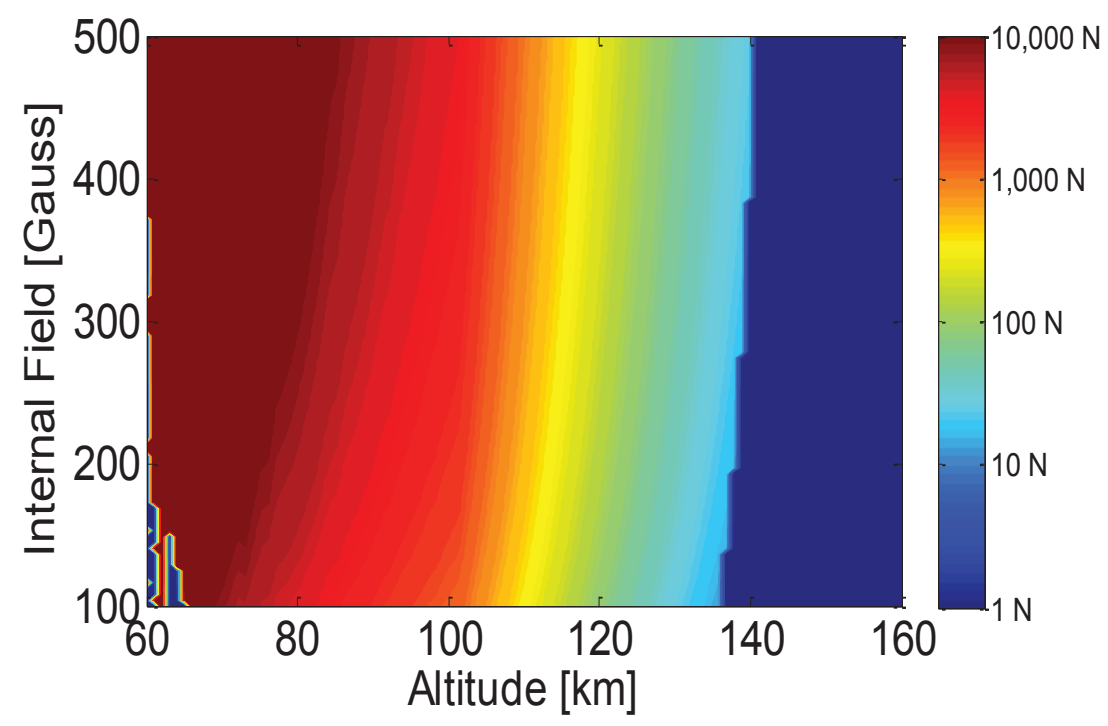

Figure 11. Martian Drag Force. Logarithmic drag force for equilibrium Magnetoshell and increasing magnetic field.

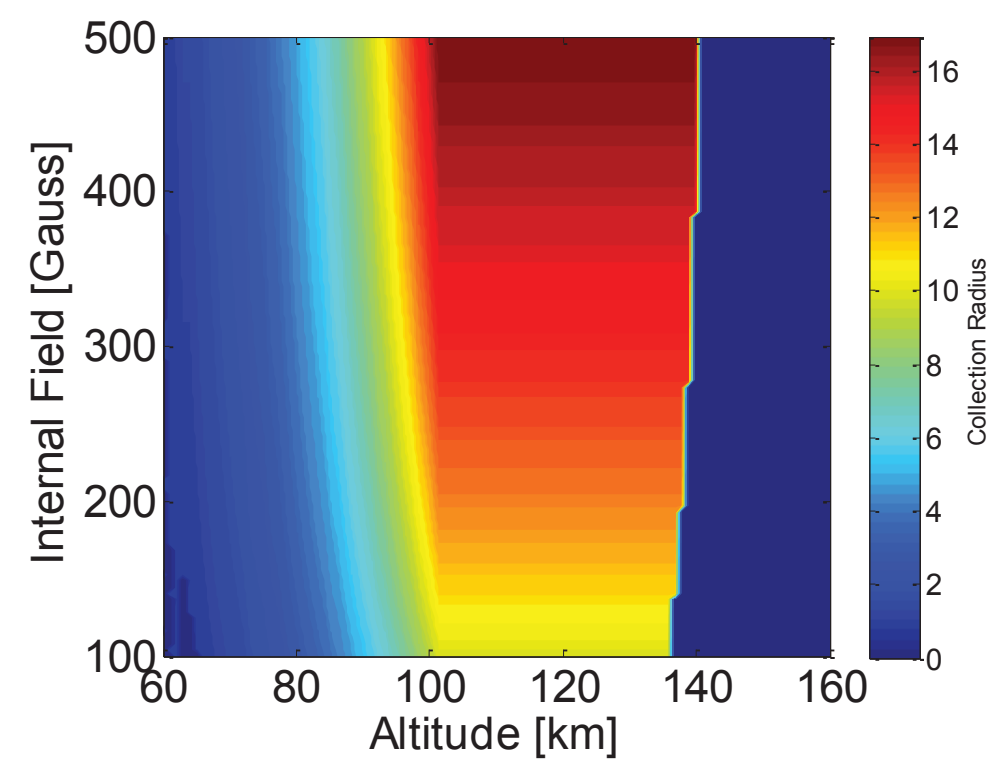

Figure 12. Effective Collection Radius. Outer radial boundary of confined CEX. 
A Plasma Aerocapture and Entry System for Manned Missions and Planetary Deep Space Orbiters

MSNW LLC

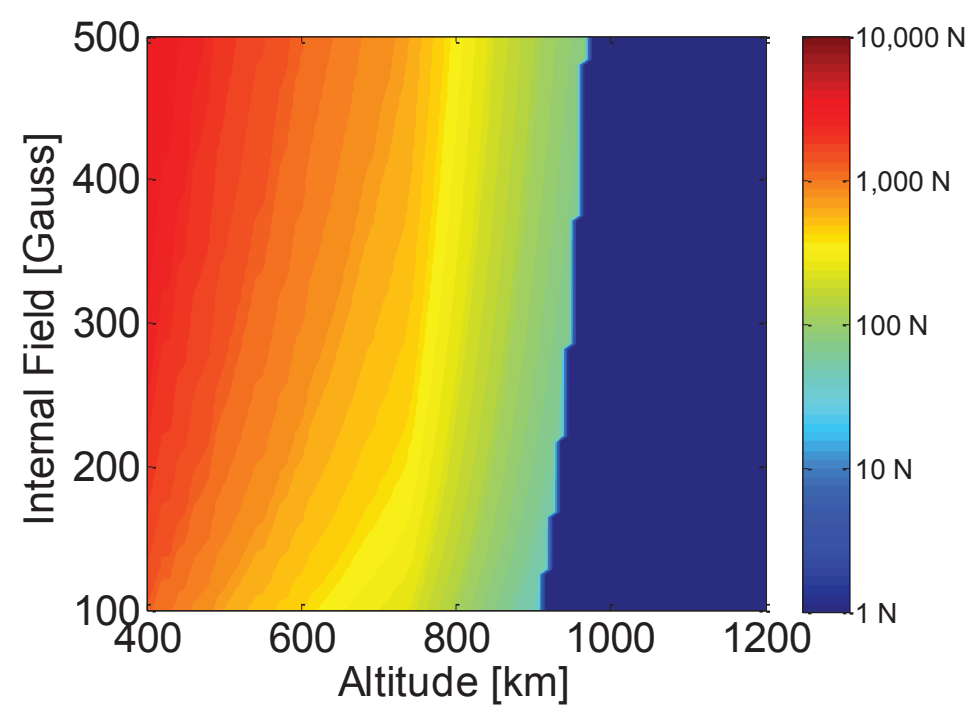

Figure 13. Neptune Drag Force. Logarithmic drag force for equilibrium Magnetoshell and increasing magnetic field.

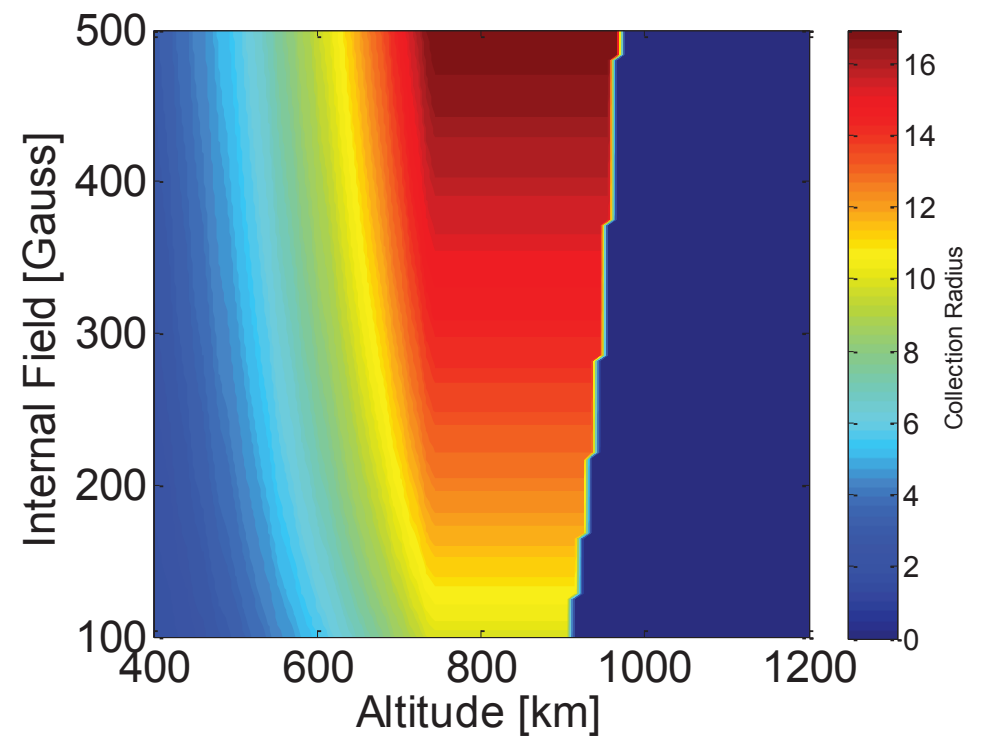

Figure 14. Effective Collection Radius. Outer radial boundary of confined CEX. 
A Plasma Aerocapture and Entry System for Manned Missions and Planetary Deep Space Orbiters

MSNW LLC

\section{Related Modeling Effort - Sel HiFi}

The Plasma Science and Innovation Center (PSI) at the University of Washington has developed a two-fuid model of interaction plasma and neutral fluids[11]. This model is based on the SEL-HiFi code [12], a 3D MHD code with advanced features that are ideal for modeling this complex interaction. SEL-HiFi uses a flux-source form for inputs, is completely implicit with an adaptive grid with high-order spectral elements. To this code Dr. Meier has added the capability to investigate a neutral fluid with resonant charge exchange, electron-impact ionization, and radiative recombination reactions. Braginskii closures are used for the plasma, and ChapmanEnskog hard sphere closures are used for the neutral gas. And while experimental validation is on-going with the latest experimental results, presented here is a summary of results obtained from early simulations runs and presented at the 2011 Innovative Confinement Concepts meeting. This code was developed for DOE fusion applications and AFRL space propulsion thrusters. Specifically, this work was used under an AFOSR program with MSNW and the University of Washington to investigate the effects of a high density magnetized plasma interacting with a background neutral gas. Interestingly, these results showed that the interaction of these gases, when the plasma and neutral conditions are in the right magnitudes and ratios can yield significant and interesting new physics. A summary of results for a space propulsion application will now be given to highlight the interaction effects and similarity to the Magnetoshells $[13,14,15]$. This code will be used in Phase II to further model the Magnetoshell interaction with a neutral gas.

Figure 15 and Figure 16 show results from a propagating Field Reverse Configuration Plasmoid (FRC) interacting with a background neutral gas. An FRC is a fully ionized, magnetized plasma with an ion and electron temperature of a 5-20 eV and plasma densities of 1E18-1E21 $\mathrm{m}^{-3}$. In these cases, the total plasma mass of the FRC and neutral mass of the interaction region are equal. Additionally, the neutral mass is centered on axis as in the experiment and extends for $50 \mathrm{~cm}$ downstream. In these simulations a 30 microgram FRC is colliding with a 30 microgram Neon region after being initialized to $26 \mathrm{~km} / \mathrm{s}$ axial velocity. Figure 15 shows the neutral density and momentum during the FRC interaction. It is clear that the FRC compresses and accelerates the neutral propulsion. Additionally, there is evidence of some ionization of the neutral gas. Figure 16 shows the plasma pressure increase as the FRC interactions with the neutral pressure. Finally, as you would expect, there is an increase in diamagnetism and a slowing of the FRC as it passes through the gas. 
A Plasma Aerocapture and Entry System for Manned Missions and Planetary Deep Space Orbiters

MSNW LLC

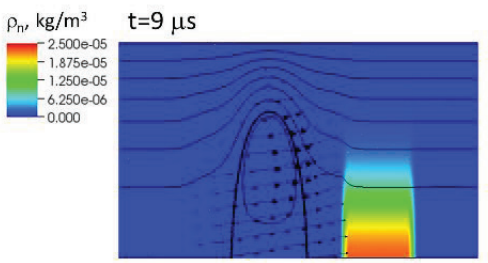

$\mathrm{t}=27 \mu \mathrm{s}$

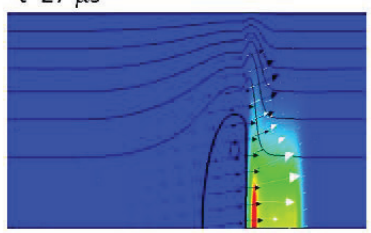

$\mathrm{t}=17 \mu \mathrm{s}$

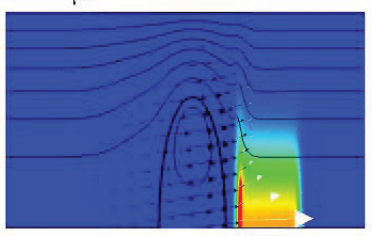

$t=36 \mu s$

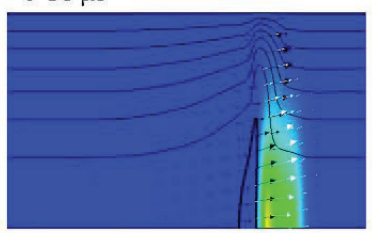

Figure 15. Neutral density psuedocolor plots for baseline thruster simulation with a Gaussian radial neutral gas profile. Black and white arrows indicate the direction and magnitude of plasma and neutral momentum, respectively. Clear neutral entrainment is seen.

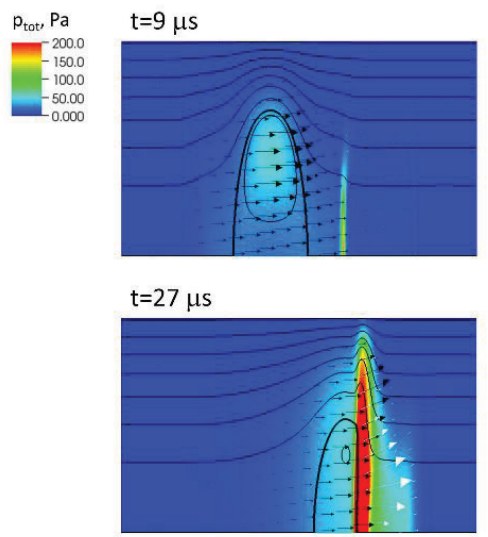

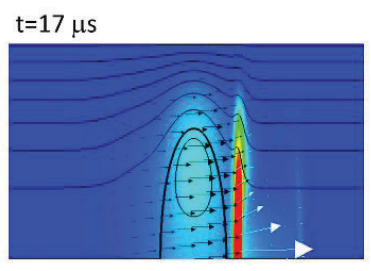

$\mathrm{t}=36 \mu \mathrm{s}$

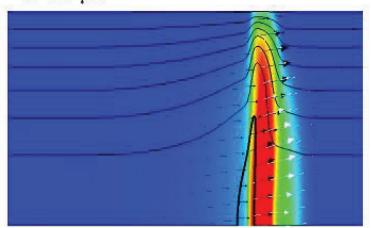

Figure 16. Pressure psuedocolor plots for baseline thruster simulation. Clear neutral entrainment, pulse sharpening, and increase in external magnetic field pressure are seen.

Figure 17 and Figure 18 show temporal profile of the total momentum and energy components of the FRC as well as the neutral population. Again, this is for a case in which the neutral mass was equal to the plasma mass. Quite obvious from these results is that both charge exchange and ionization play a critical role in these processes and that momentum can successfully be transferred to the neutral gas as desired. For an equal plasma to neutral mass, approximately $50 \%$ of the momentum of the plasma is transferred to the plasma, with a majority of that momentum transfer coming from charge exchange. In these cases, it appears that there is significant ionization of the background plasma although that energy appears to mostly come from the initial thermal energy of the plasma rather than the kinetic energy, exactly as desired. And while not a loss mechanism in neutral entrainment as the new plasma will be accelerated with the initial FRC plasma, this is less neutrals entrained overall, lessening the efficiency and T/P gain. Additionally, it was found that the amount of ionization was directly the result of the temperature of the final, expanded translating FRC. At very high temperatures $(20 \mathrm{eV}$ or above) 
A Plasma Aerocapture and Entry System for Manned Missions and Planetary Deep Space MSNW LLC

Orbiters

it was found that $90 \%$ of neutrals may be ionized in entrainment. For $5 \mathrm{eV}$ total temperatures (similar to experiments) and high downstream neutral density it was found that as low as $20 \%$ of neutrals are ionized while $80 \%$ of the plasma momentum is transferred to the neutrals.
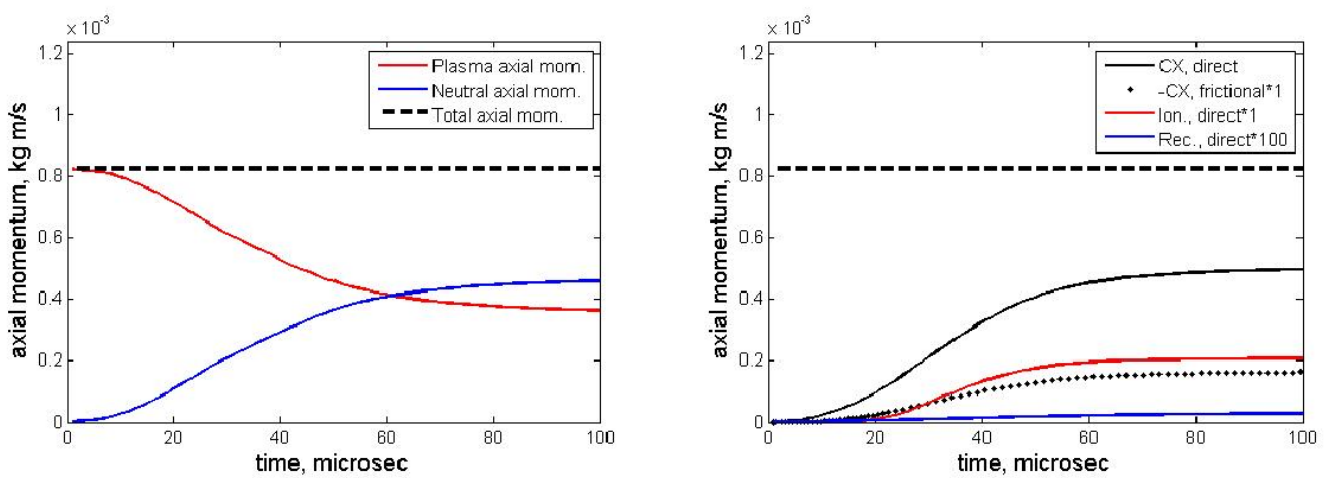

Figure 17. Temporal profiles of momentum-related quantities for ELF baseline simulation
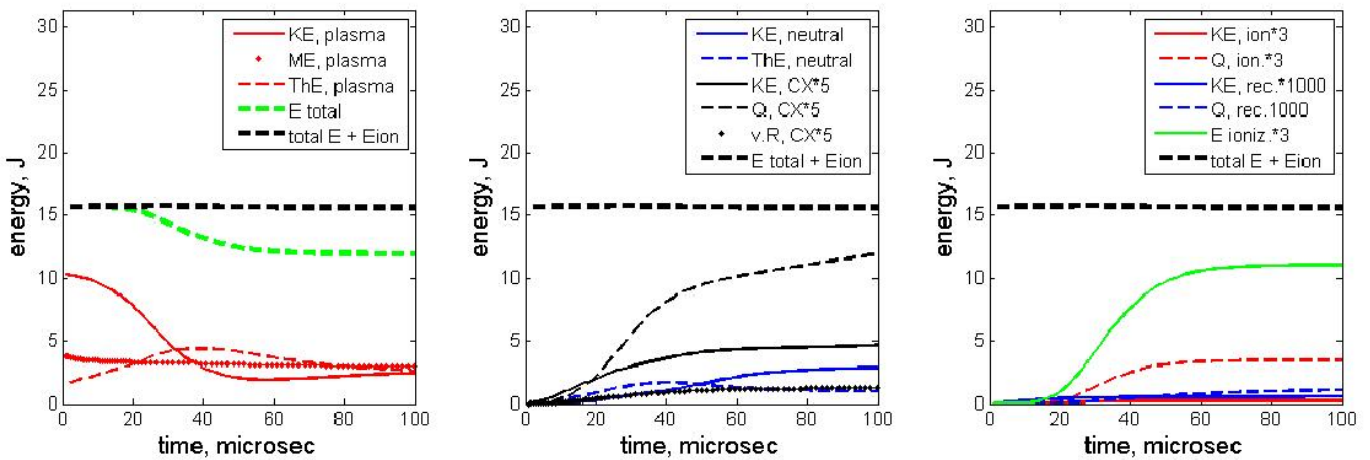

Figure 18. Temporal profiles of energy-related quantities for thruster baseline simulation 
A Plasma Aerocapture and Entry System for Manned Missions and Planetary Deep Space Orbiters

MSNW LLC
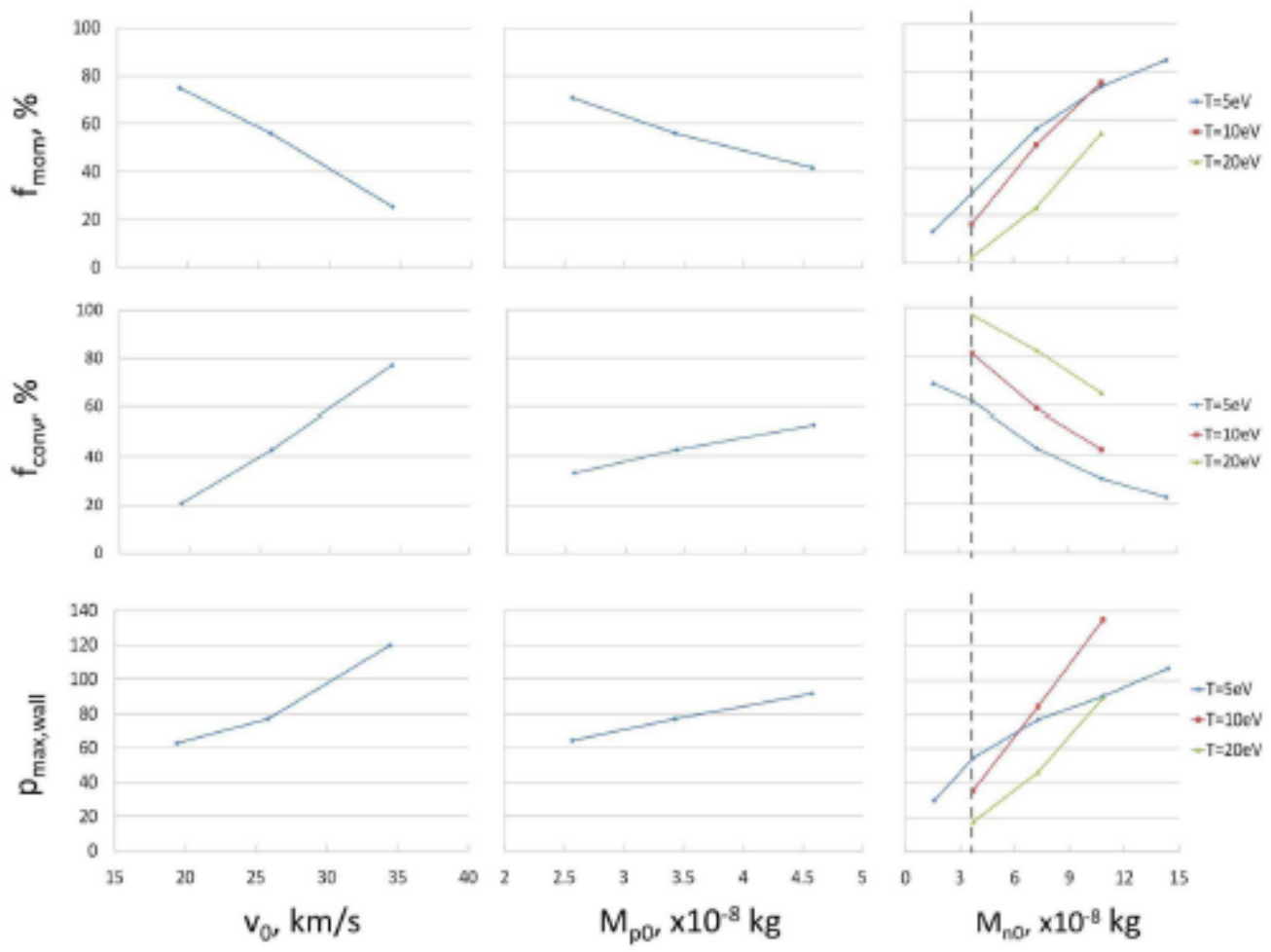

Figure 19. Metrics for thruster simulations with Gaussian neutral gas profiles. Variations in the three metrics, the fraction of momentum transferred, the ionization fraction, and the maximum total ion plus neutral pressure at the radial wall are shown with respect to plasma axial speed, initial plasma mass, initial neutral mass, and initial FRC temperature. 
A Plasma Aerocapture and Entry System for Manned Missions and Planetary Deep Space Orbiters

MSNW LLC

\section{Laboratory Demonstration of an Argon Magnetoshell and Drag}

The demanding requirements of a ground test for a Magnetoshell system, put very specific constraints on the neutral gas injection, Magnetoshell, and vacuum facility. In order to realistically simulate the interaction of an atmospheric flowing gas with a large, magnetized plasma-filled dipole several design parameters must be met.

- Neutral gas and plasma densities of the neutral jet and Magnetoshell need to be in the collisional regimes of interest, namely $>1 \mathrm{E} 18 \mathrm{~m}^{-3}$. Goal densities of $1 \mathrm{E} 19 \mathrm{~m}^{-3}$ were chosen.

- Chamber effects must be minimal, i.e. background pressure less than $1 \mathrm{E} 18 \mathrm{~m}^{-3}$. Goal background densities of $1 \mathrm{E} 17 \mathrm{~m}^{-3}$, i.e. $1 \mathrm{E}-5$ Torr was chosen to prevent artificial damping drag.

- Neutral jet interaction must supersonic

- Magnetoshell interaction time must be longer than the magnetic trapping time and radial diffusion timescales

- Chamber wall interaction must be minimal

These requirements show that the required vacuum pumping and chamber size for steady operating is prohibitive. A flowing argon gas at $1 \mathrm{e} 19 \mathrm{~m}^{-3}, 1 \mathrm{E}-5$ Torr, 1 meter diameter, and 323 $\mathrm{m} / \mathrm{s}$ requires $5700 \mathrm{sccm}$ gas with $7 \mathrm{ML} / \mathrm{s}$ of pumping. However, a pulsed system can easily handle this requirement as long as the time of interest is less than the neutral transit time of the chamber, or approximately $21 \mathrm{~ms}$ for cold neutrals or $2.1 \mathrm{~ms}$ for high velocity neutrals. Therefore, the $3.3 \mathrm{~m}$ long, $2.1 \mathrm{~m}$ diameter MSNW vacuum facility was used to test the Magnetoshell and neutral gas injector in a pulsed manner. The impulse was measured on the MSNW torsional thrust stand, which has been shown to be able to resolve approximately 100 $\mu \mathrm{N}-\mathrm{s}$.

\section{Magnetoshell Setup}

Shown in Figure 20 - Figure 23 is the Magnetoshell simulator that was tested during this reporting period. This consists of a 200 -turn magnetic dipole with a two-phased, spherical, RF antenna. The RF antenna are low resistance Litz wire encased in a Tef-Zel high temperature insulator. The entire assembly is fed with low-inductance, low-resistance strip line with a silicone high temperature insulation layer. It is mounted on a G-10 mount that places the antenna midway between the thrust stand platform and the top of the chamber. This positioning allows the maximum interaction with a 15-degree half angle expanding neutral jet.

A thruster power-processing unit (PPU) was used for this experiment consisting of a pair of IGBT switches, high-efficiency capacitors, and all required charging and fiberoptic triggering. This system, shown in Figure 22 was operated at $160 \mathrm{kHz}$ and $1.6 \mathrm{kV}$. DC charging power and fiber-optic signals are transmitted through an array of small (low-spring constant) feed cabling on the stand. Gas is injected along the axis and fed by a Parker Series-9 puff valve backed by an Alicat $500 \mathrm{sccm}$ flow controller. Pre-ionization and discharge initiation uses a small dielectric 
A Plasma Aerocapture and Entry System for Manned Missions and Planetary Deep Space Orbiters

\section{MSNW LLC}

surface flashover discharge to initialize the plasma formation and provide a seed electron population. Gas is injected into the center of the Magnetoshell and will be discussed further in the testing section of this report.

Figure 20 shows the dielectric thrust stand to be used in Magnetoshell testing. Figure 21 shows a close-up of the antenna setup. Figure 22 shows an external, angled view of the Magnetoshell. In this photo, the G-10 mount is bolted to the torsional thrust stand platform. The downstream 3-axis linear probe array can be seen. Figure 23 shows the face-on view from the perspective of the neutral injector. In this photo, a double Langmuir probe with $10 \mathrm{~mm}^{2}$ ion collection electrode, 100-turn magnetic field probe, and Retarding Potential Analyzer are seen. Downstream plasma and magnetic field probes on a 3 axis translation stage was used to measure the average ion (momentum) and electron (flux) radial profiles of the Magnetoshell.

MSNW has developed a dielectric torsional thrust stand that will be used to measure the drag impulse of the Magnetoshell. A G-10 thrust stand has been designed to integrate with the MSNW MVF. This thrust stand is an entirely non-magnetic and non-inductive torsional thrust stand that was designed to enable the accurate testing of a pulsed inductive thruster. The G-10 thrust stand uses a Philtec D63 fiber-optic displacement sensor for isolated, high-accuracy position measurements, a pulsed and steady calibration system, and passive eddy-current damping. This stand was calibrated using the MSNW standard gasdynamic calibration cold gas thruster and a traditional DC free weight system during these tests. 
A Plasma Aerocapture and Entry System for Manned Missions and Planetary Deep Space Orbiters

\section{MSNW LLC}
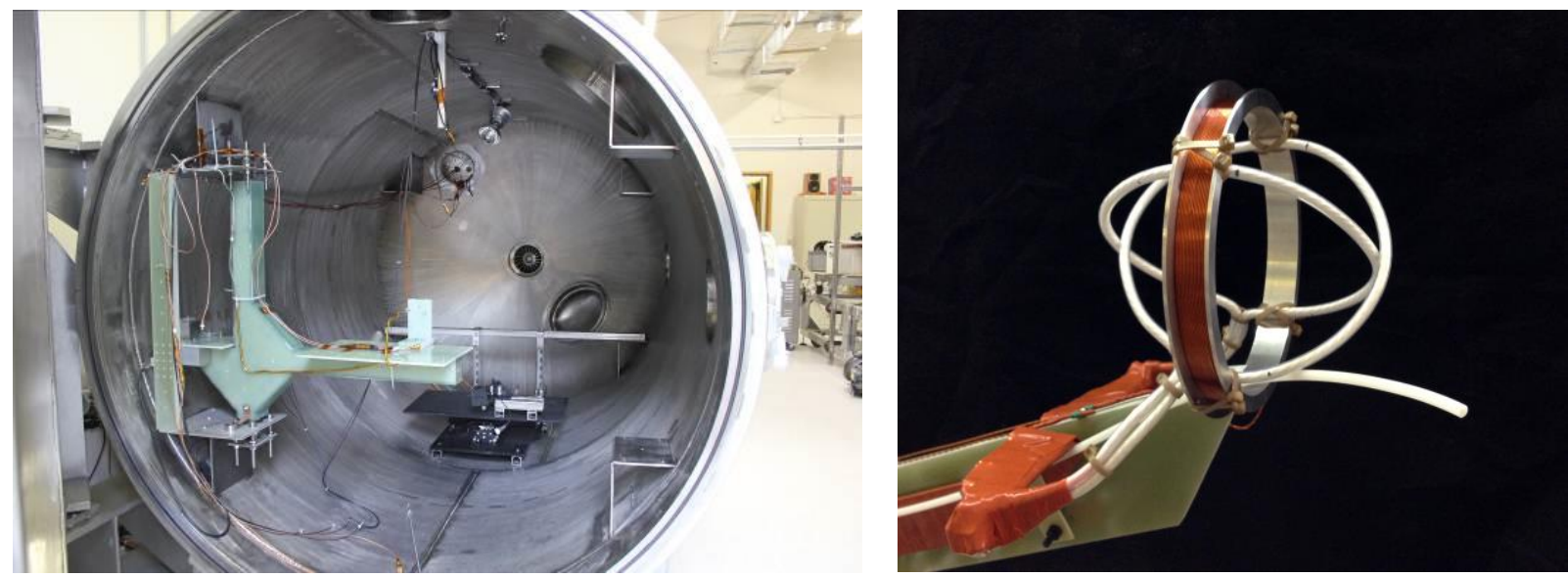

Figure 20. 2 m MSNW vacuum chamber with dielectric, asymmetric torsional thrust stand.

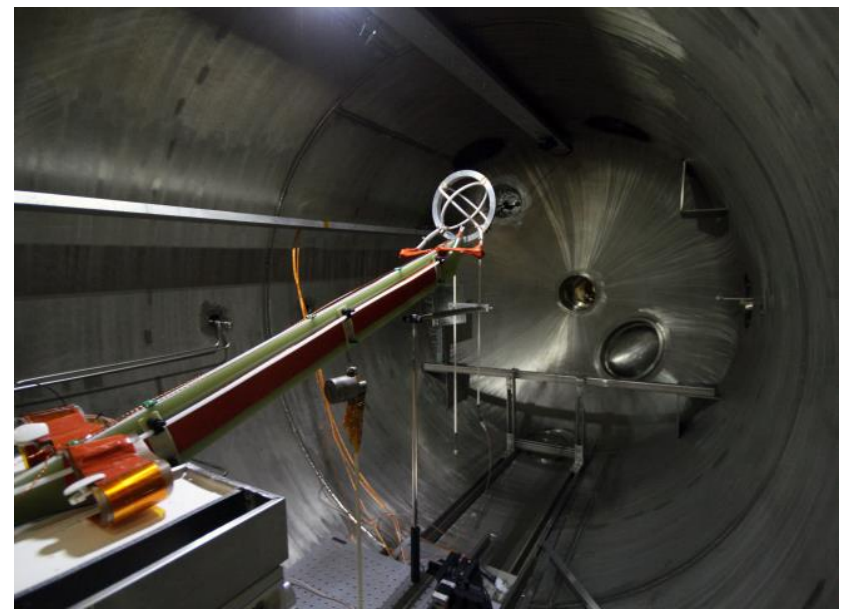

Figure 21. 200-turn dipole and 2-phase RMF antennas oriented perpendicular to neutral flow.

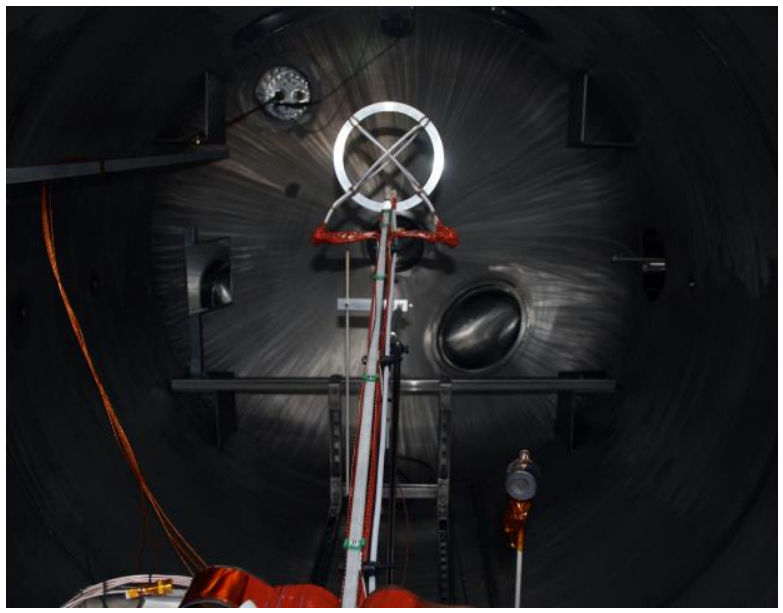

Figure 22. Magnetoshell installed in chamber. Figure 23. Magnetoshell installed in Shown are two in-chamber power supplies (potted), low-inductance stripline, G-10 mount.

chamber. Shown are Magnetoshell antenna, bias field magnet, downstream Langmuir probe, magnetic field probe, and RPA. 
A Plasma Aerocapture and Entry System for Manned Missions and Planetary Deep Space Orbiters

MSNW LLC

\section{Pulsed Arcjet}

The original intent of this program was to design and build a high-energy, pulsed arcjet to provide a supersonic (or greater than supersonic) velocity neutral jet. For these Phase I tests, argon gas/propellant is to be used. Argon has similar atomic mass to a Martian atmosphere (Carbon Dioxide) and has well-understood kinetic, ionization, and charge exchange cross sections. This experimental effort was not successful, and another technique (MPD with entrainment) was employed and will be detailed in the next section. For completeness, a short discussion on the pulsed arcjet will now be given.

The Arcjet design used in this program is a functional replica of the $1 \mathrm{~kW}$ NASA, "Low Power Arcjet", in this case with a $0.6 \mathrm{~mm}$ diameter tungsten constrictor and 30 degree nozzle. This steady thruster was shown to operate reliably, without significant erosion, and with high performance. MSNW designed and manufactured a modified version of this arcjet with a $0.7 \mathrm{~mm}$ throat and tested with both an EDM (electrical discharge machined) tungsten nozzle and a graphite nozzle. The arcjet was initiated with a $3 \mathrm{kV}$, pulsed starting circuit. It had two modes of operation that were tested, the steady mode was powered by a $200 \mathrm{~V}$ DC power supply operating at 1-20 Amps. This thruster was operated from 100-500 $\mathrm{sccm}$ of Argon and showed expected operation and performance in a steady manner.

It was then operated in a pulsed mode, in an attempt to limit the gas flow to the relevant timescales. The arcjet was powered with a $1.35 \mathrm{mF}$, IGBT-switched capacitor bank charged to 200-1600 Volts. The results were conclusive and insufficient for this program. While it was possible to pulse the arcjet with $100+\mathrm{ms}$ pulses (gas, electrical energy, or both), it was not possible to form $1 \mathrm{~ms}$-scale discharges that were repeatable. It is important to note that for these tests, a limiting resistor of 3-100 Ohms was used to force the thruster into an arcjet mode (rather than a much higher current MPD mode). In Figure 24, the primary concern is seen. A startup transient is developed during the first several hundred microseconds of the discharge in which high velocity, discrete effluvia are emitted from the thruster. If the discharge is extended for several milliseconds, as shown in Figure 25, a plume of superheated, partially-ionized, accelerated argon is seen. However, shot-to-shot variability for this discharge was enormous and not deemed useful. Figure 26 shows an argon jet in a long duration pulse discharge. Above 10's of miliseconds the jet stabilized and became repeatable. Figure 27 shows the arcjet, gas feed, and electrical hookups as tested. In conclusion, while a suitably sized and powered arcjet was developed and tested that appeared to generate high velocity, mostly neutral argon, it was not repeatable or reliable enough for these tests. 
A Plasma Aerocapture and Entry System for Manned Missions and Planetary Deep Space Orbiters

MSNW LLC

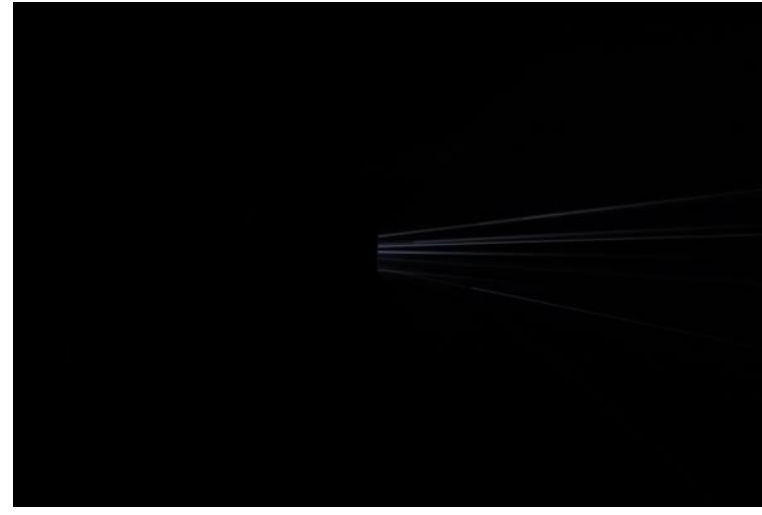

Figure 24. Arcjet Startup Transient.

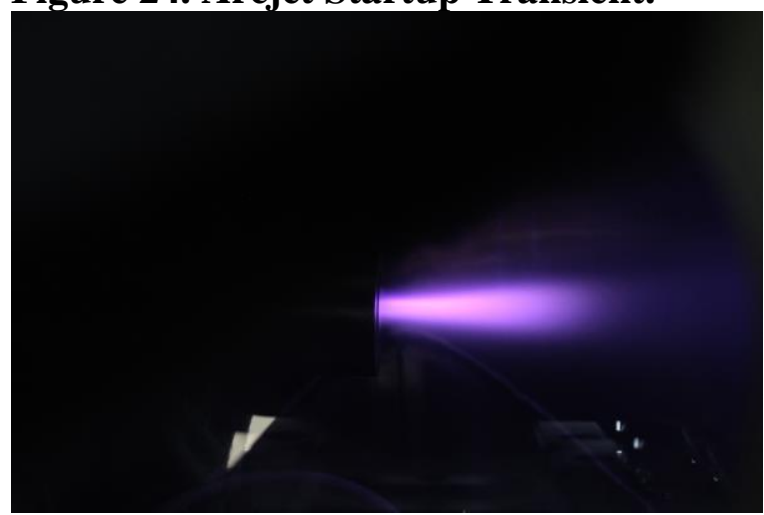

Figure 26. Arcjet pulsed operation - 100 ms.

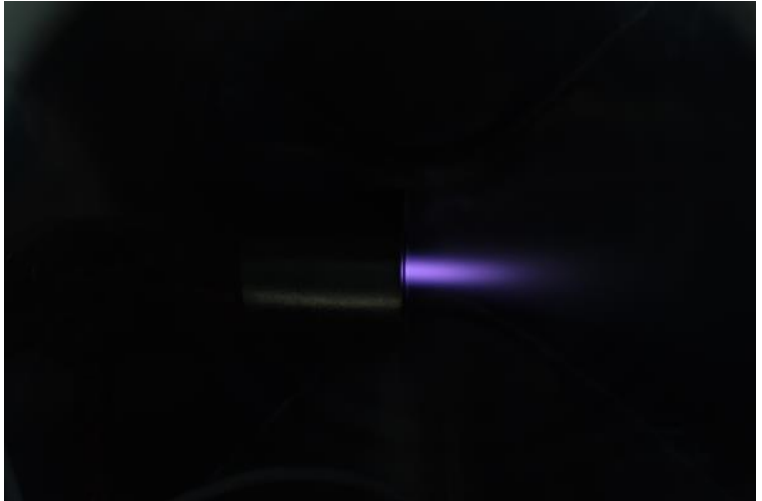

Figure 25. Arcjet pulsed operation - 1 ms.

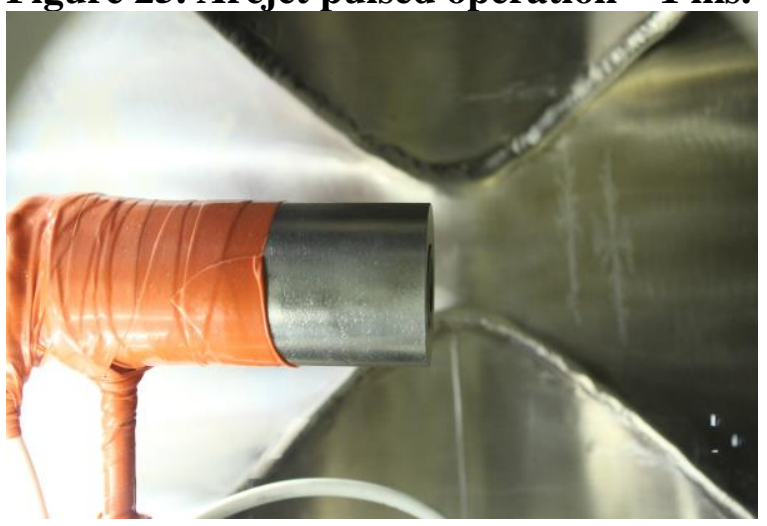

Figure 27. Arcjet mounted in the 'Junior' test facility.

\section{MPD Jet Accelerator}

An MPD (Magnetoplasmadynamic) accelerator/thruster was then designed and built to generate the required jet. In a traditional MPD (unlike an arcjet) the majority of the gas is ionized and accelerated via electromagnetic forces rather than thermal forces, i.e. no thermal nozzle. In practice, MPD's will have a mix of neutral and plasma flow which is all swept into one somewhat ionized jet. The ionization fraction of the jet is a specific function of the electrical power and input flow rates. For this program a miniature MPD was designed and built to accelerate argon to high velocities. It was then operated in an over-gased manner in which neutral gas is ejected ahead of the plasma jet. As the higher velocity plasma transitions through the neutral gas, the high-density neutrals are accelerated through charge-exchange and viscous processes. Due to the collisionality of these gases, it is believed that the resultant plasma velocity (after being decelerated) was similar to the velocity of the neutrals. The exact ionization fraction is unknown and will have some effect on the Magnetoshell as will be described later. For demonstrating and calculating the relative amount of trapping by the Magnetoshell, this technique was successful.

The MPD tested has a $3.8 \mathrm{~mm}$ outer diameter tungsten inner anode with a $5.8 \mathrm{~mm}$ inner diameter, 15-degree outer cathode. The cross sectional area is equivalent to a $4.3 \mathrm{~mm}$ orifice. It 
A Plasma Aerocapture and Entry System for Manned Missions and Planetary Deep Space Orbiters

\section{MSNW LLC}

was operated with a Parker Series-9 puff valve and a 90 psig argon backpressure. It was powered by a $1.2 \mathrm{kV}, 1.3 \mathrm{mF}$ capacitor with a switched IGBT that allowed for discrete, fixed pulses. Gas delivery is provided by a standard MSNW high speed puff valve driver. Figure 28 shows a photograph of the MPD mounted in the MSNW facility, approximately 1.5 meters from the Magnetoshell. Additionally, a curved, elliptical mirror provides complete optical overage of the chamber. Figure 30 shows a 2 ms-wide MPD discharge current. At the end of the triggered discharge, the MPD current is terminated by opening the snubber-circuitry-protected IGBT. Figure 31 shows the ion saturation current for a double Langmuir probe located approximately 10 $\mathrm{cm}$ downstream of the Magnetoshell. Ion velocity for the MPD was measured with the average time of arrival of a $1 \mathrm{~ms}$ MPD discharge at $1.2 \mathrm{kV}$ and a $1 \mathrm{~ms}$ gas puff. This showed that with a standard discharge, the average ion velocity was $1700 \mathrm{~m} / \mathrm{s}$. When a neutral gas is added with a 10 ms puff (MPD still triggered at time $=0$ seconds), the average velocity decreased to $830 \mathrm{~m} / \mathrm{s}$, though the velocity at impact is likely significantly slower as the deceleration happens the entire length of the travel region. Additionally, the pulse is wider, both in time and radially suggesting significant entrainment. Peak ion densities were 9E17 (+/- 3E17) $\mathrm{m}^{-3}$. Neutral densities will be determined from thrust stand data. 
A Plasma Aerocapture and Entry System for Manned Missions and Planetary Deep Space Orbiters

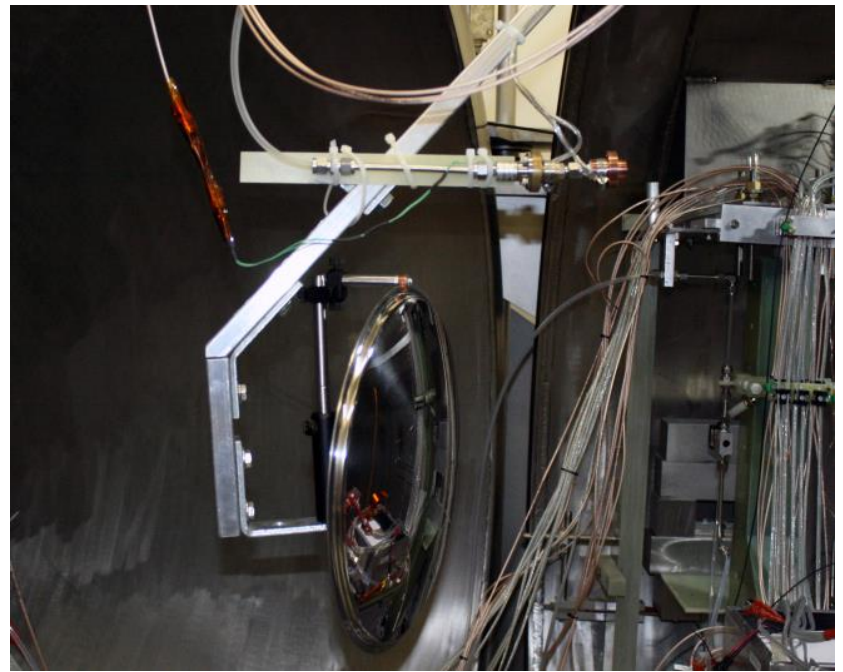

Figure 28. MPD mounted in the MSNW vacuum facility. Shown is the MPD with integrated puff valve, spherical mirror, and in the background, the 'waterfall' power feed lines.

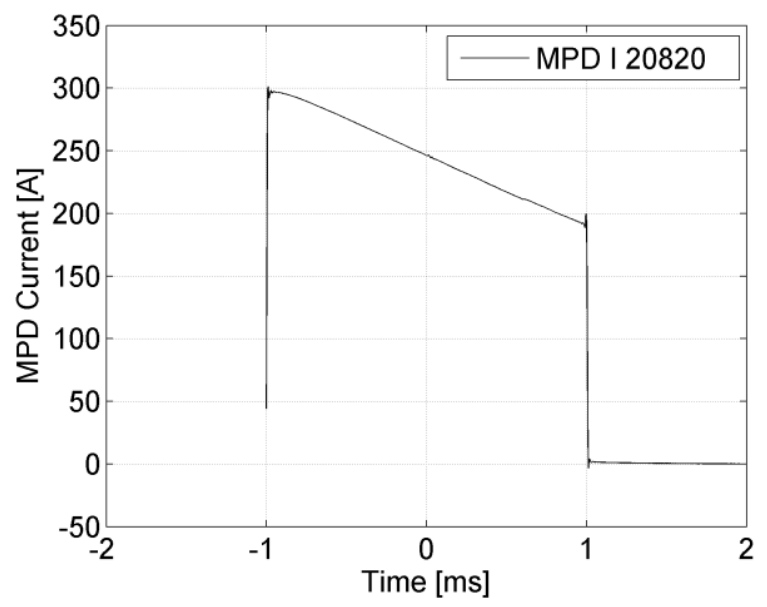

Figure 30. MPD current with a $1 \mathrm{kV}$ charge voltage.

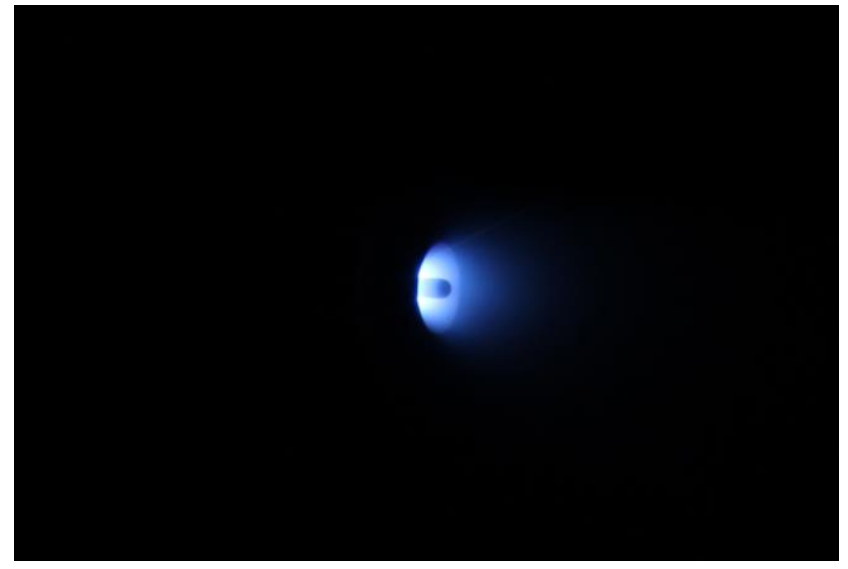

Figure 29. Pulsed MPD operating with an argon propellant and $2 \mathrm{~ms}$ discharge.

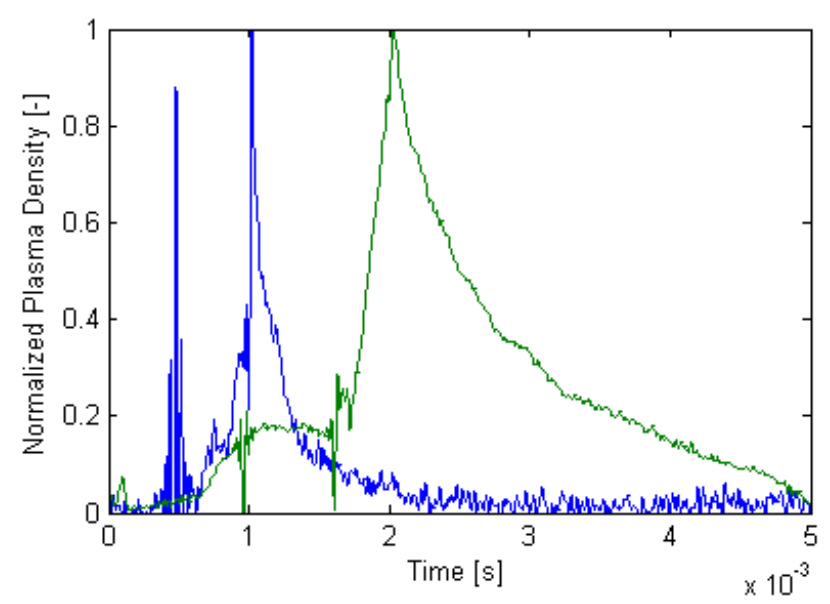

Figure 31. MPD downstream plasma density with and without a neutral pre-gas as blue and green, respectively. 
A Plasma Aerocapture and Entry System for Manned Missions and Planetary Deep Space Orbiters

MSNW LLC

\section{Magnetoshell Discharge Testing}

Significant progress was made during this Phase I with a successful, complete subscale system test for Phase I. Figure 32 shows the Magnetoshell as mounted in the MSNW chamber. This photograph was taken while under vacuum through a side window and with an elliptical 'safety' mirror. The bright light in the photo is the chamber LED illumination, and the perspective is shown from below the MPD. The mirror and assembly is shown in Figure 28. Figure 33 shows a high-power MPD discharge into the chamber. In this configuration the MPD and neutral puff valve are located 1.5 meters from the center of the Magnetoshell antenna with the MPD central axis aligned with the antenna. As the high velocity neutrals impact the Magnetoshell mounting and antenna a net impulse is imparted to the Magnetoshell, its rigid G-10 support arm, and thus, the torsional stand. This impulse deflects the stand, which oscillates with a 9.6 second period. Displacement is measured by an optical sensor at the end of the torsional arm. The stand is counterbalanced to reduce secondary oscillations and includes a passive eddycurrent damping system. Calibration is completed in air with a standard DC, weight calibration system. In vacuum calibration is accomplished with a standardized, AFRL-calibrated cold gas thruster which is fed a steady Xenon gas flow. By measuring the deflection and comparing with externally-validated thrust stand results, the thrust stand spring and deflection constants can be measured.

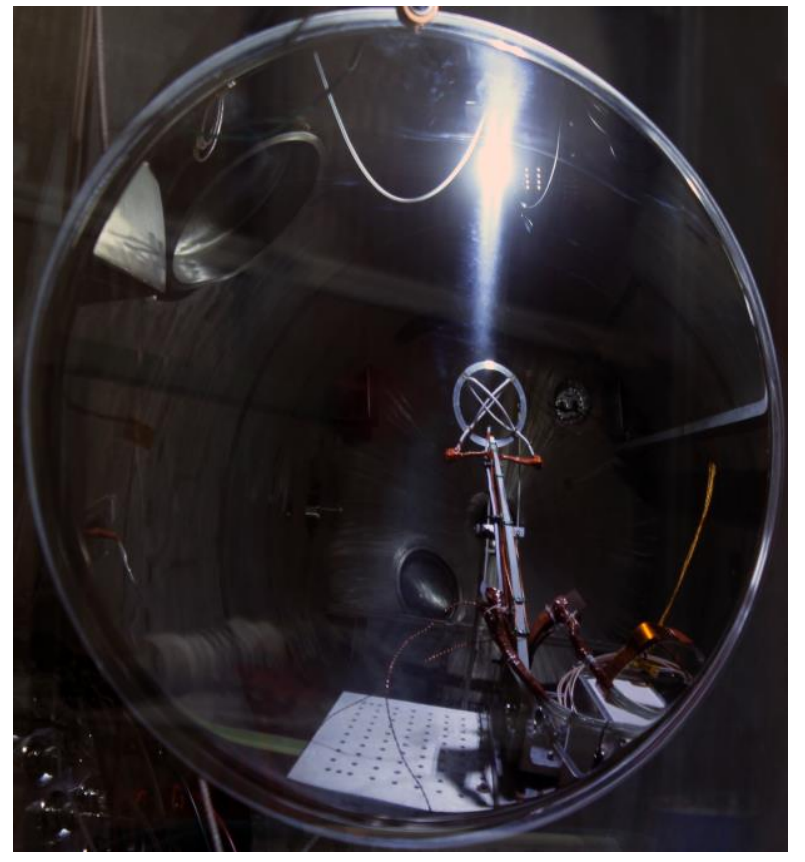

Figure 32. Magnetoshell mounted in the MSNW vacuum facility. Shown is the Magnetoshell on the thrust stand. Shown are the in-chamber PPU circuitry, power and gas feeds and full MSNW chamber. Light in background is the chamber LED illumination.

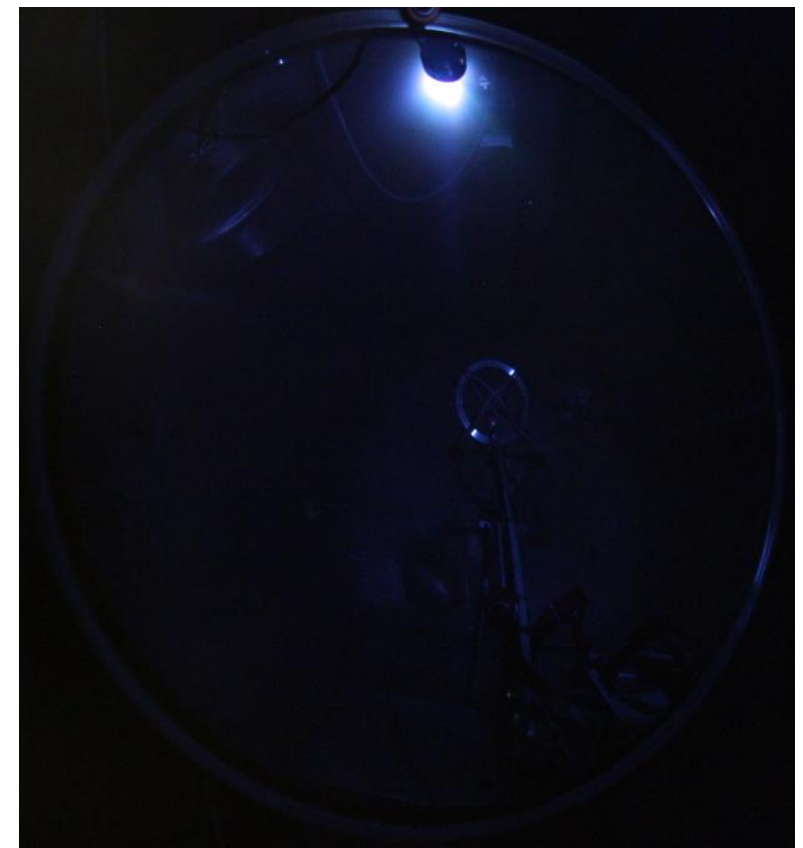

Figure 33. Pulsed MPD operating with an argon propellant and $\mathbf{2}$ ms discharge. Shown is 90 psig, $1 \mathrm{~ms}$ argon puff at 400 Amp peak discharge. 
A Plasma Aerocapture and Entry System for Manned Missions and Planetary Deep Space Orbiters

MSNW LLC

The Magnetoshell was tested with an Argon gas under a variety of operating and gas flow conditions. For this technical report a summary of the initial results will be given. In Figure 34, a 3 Joule RF discharge creates a plasma discharge, entirely out of incoming neutral flow. In this case, a $30 \mathrm{~ms}$ neutral gas puff flows into the Magnetoshell antenna. A discharge is initiated with a 1 Joule surface flashover discharge, creating a uniform argon discharge. The RF ringing lasts approximately 40 microseconds with a decaying ring-down. In this case the gas is entirely sourced from the ambient, flowing neutrals. While this may be an optimal method for operating in the space environment it requires that a significant portion of the neutral gas puff pass through and past the antenna region before a discharge can be formed and maintained. Therefore, a small internal gas feed provided gas injected towards the center of the Magnetoshell with a small 0.25 " injector located at the magnet edge. Propellant is fed to the Magnetoshell constantly with a small, $50 \mathrm{sccm}$ flow rate, raising the chamber background pressure from $3 \mathrm{E}-7$ Torr to $1 \mathrm{E}-5$ Torr, the upper limit of desired operation. Note, this is only $10 \%$ of the desired full Magnetoshell density, but was sufficient to provide seed neutral gas at the required position prior to neutral flow simulator initiation. Figure 35 shows an incoming, slow neutral gas puff, however now with an internal gas flow. This showed a clear increase in ionization rate, magnetized plasma formation, and plasma density at the edge of the antenna. Adding an internal neutral gas flow also allowed the initial jet puff to be timed so that the Magnetoshell RF was operated during the arrival of the leading edge of the puff gas flow, rather than waiting until sufficient density populated the antenna region.

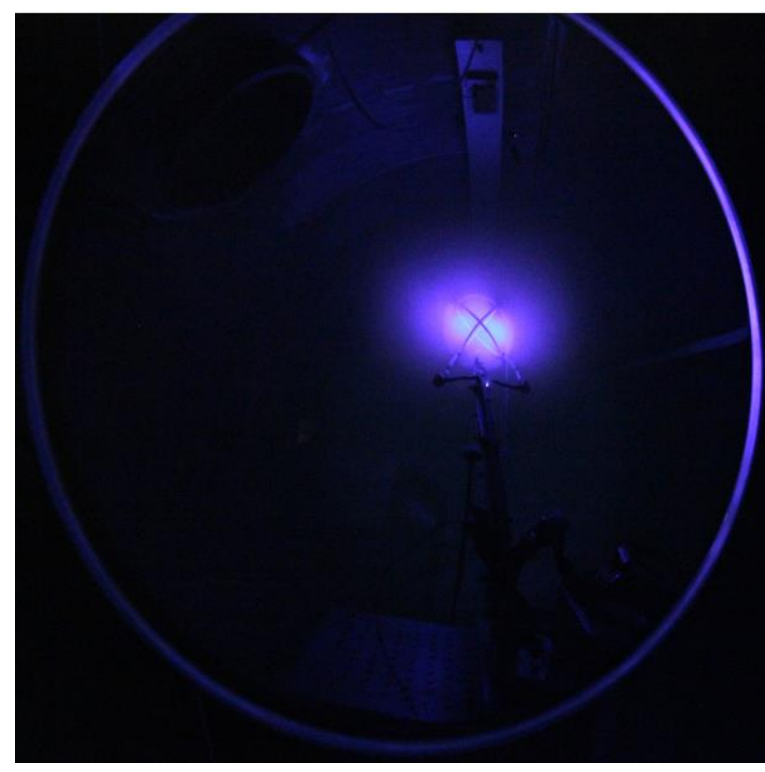

Figure 34. Magnetoshell operating and intercepting a partially ionized jet. Shown is a 3 Joule RF and $30 \mathrm{~ms}$ neutral gas puff. . Shot \#20179

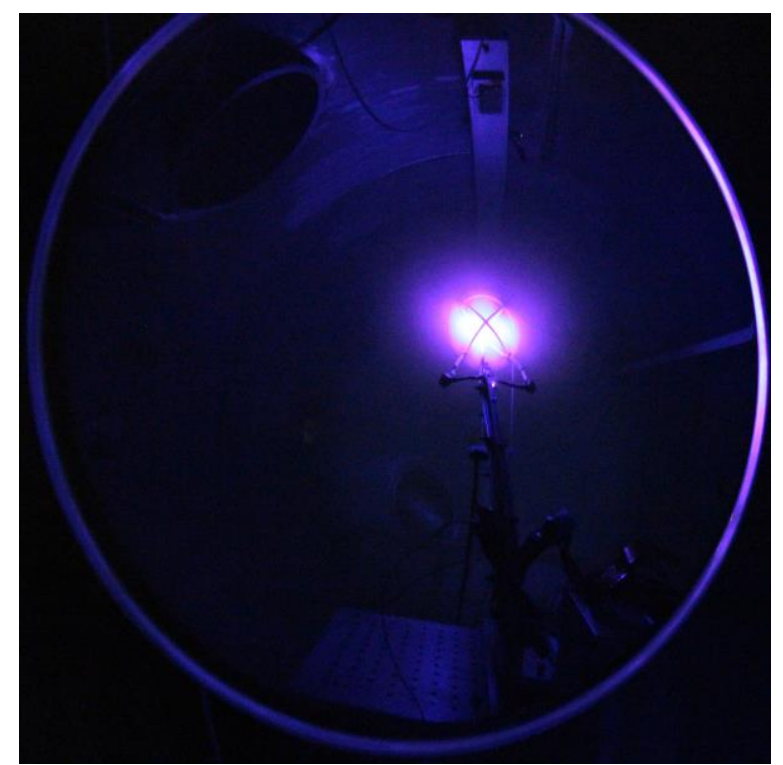

Figure 35. Magnetoshell operating with internal gas field and intercepting a partially ionized jet Shown is $50 \mathrm{sccm}$ internal feed, 3 Joule $R F$, and 20 ms neutral gas puff. Shot \#20178

Figure 36 shows the addition of the MPD. This testing had an internal gas feed, 200 
A Plasma Aerocapture and Entry System for Manned Missions and Planetary Deep Space Orbiters

\section{MSNW LLC}

Gauss bias field, and a high power MPD jet. In this case, only MPD plasma impinges on the Magnetoshell. Results were as expected, with much more in common to the past Magnetosphere work. Fully ionized, low-density, magnetized RF plasma was formed. Only high-velocity plasma was provided to the antenna by the MPD and resulted in no apparent change in discharge brightness or impulse, though there was significant increase in plasma content at the edge of the antenna region. It is believe that as the mean free path between these two low density plasma was only marginally collisional at these low plasma densities that no significant interaction occurred during the geometric interaction region of the Magnetoshell.

Figure 37 shows the full, optimized case in which the Magnetoshell is created with a low density flowing internal argon gas at $50 \mathrm{sccm}$ and on the order of $1 \mathrm{E} 17 \mathrm{~m}^{-3}$ density. The RF is discharged approximately $2 \mathrm{~ms}$ after the initiation of the MPD at when the plasma and neutral entrained jet arrives at the Magnetoshell. This timing led to the maximum increase in impulse. Additionally, the neutral jet is puffed $20 \mathrm{~ms}$ before the RF initiation, allowing the neutral front to arrive at the RF antenna. In this way the gas is swept up by the plasma jet and the higher velocity, partially ionized propellant impinges on the Magnetoshell.

By measuring the increase in the deflection of the torsional thrust stand, instantaneous impulse could be measured. By comparing the average exposure time of the stand to the various impulses, the approximate average drag could be determined. These were repeated 5 times per discharge condition to establish error and reliability percentages and are shown below. Additionally, displacement of the stand from electromagnetic, gas feed, etc was shown to be negligible for these operational geometries. A high velocity low ionization fraction gas jet has been impinged on an RF-produced Magnetoshell. This Magnetoshell demonstrated a dramatic increase in effective drag over a non-ionized, non-magnetized interaction case. 
A Plasma Aerocapture and Entry System for Manned Missions and Planetary Deep Space Orbiters

MSNW LLC

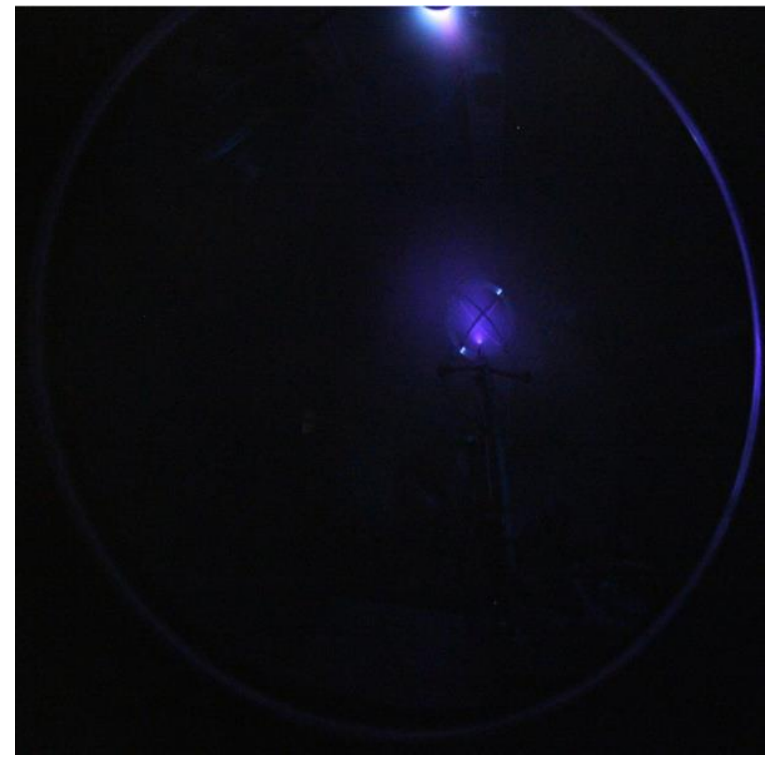

Figure 36. Magnetoshell operating with internal gas feed and intercepting only MPD plasma. Shown is 50 sccm internal feed, 3 Joule RF, and $1 \mathrm{~ms} M P D$ discharge at $1.2 \mathrm{kV}$ and 1 ms delay.\#20165

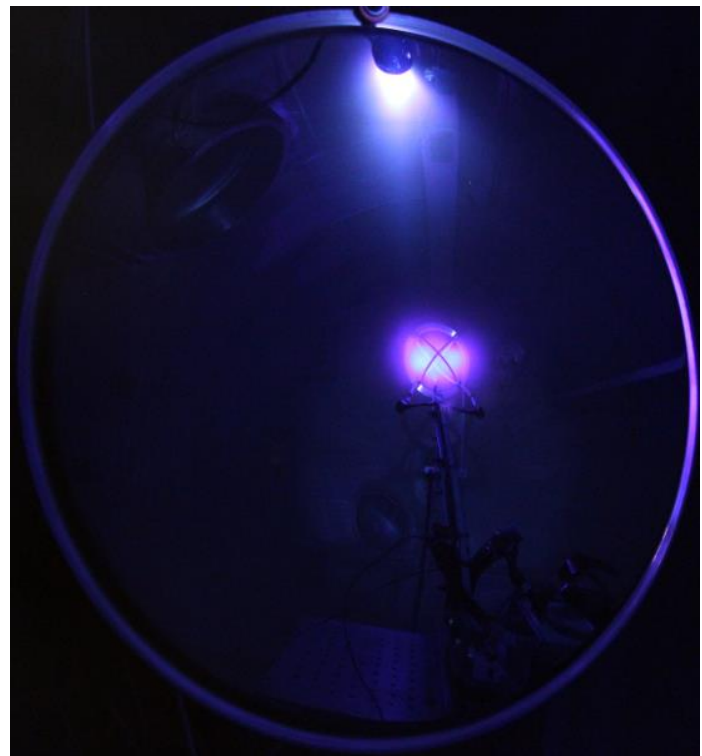

Figure 37. Magnetoshell operating with internal gas feed and intercepting an accelerated neutral and plasma jet. Shown is 50 sccm internal feed, 3 Joule RF, and $1 \mathrm{~ms}$ MPD discharge at $1.2 \mathrm{kV}$ following a $20 \mathrm{~ms}$ neutral puff. Shot \#20198

\section{Conclusions of the Experimental Validation}

An argon Magnetoshell was formed and a high velocity jet was impinged upon it. The jet impinging on the Magnetoshell hardware, dipole field, and magnetized plasma field all produced drag of interest. Shown in Figure 38 are the conditions that were tested, showing jet timing (duration), MPD duration, flow rate inside of the Magnetoshell, dipole magnetic field strength on axis, and RF plasma generation and injection.

\begin{tabular}{|lccccc|}
\hline \multicolumn{1}{r}{ Test Condition } & Neutral Jet & MPD & Internal Flow & Dipole Field & RF \\
\hline MPD & $0-30 \mathrm{~ms}$ & $1-5 \mathrm{~ms}$ & $0-50 \mathrm{sccm}$ & - & - \\
\hline Jet & $0-30 \mathrm{~ms}$ & - & - & - & - \\
\hline Magnetoshell & - & - & $0-50 \mathrm{sccm}$ & $0-500 \mathrm{Gauss}$ & $150 \mathrm{kHz}, 1.0-1.6 \mathrm{kV}$ \\
\hline Dipole Field and Jet & $0-30 \mathrm{~ms}$ & $1 \mathrm{~ms}$ & - & $0-200 \mathrm{Gauss}$ & - \\
\hline Magnetoshell and Jet & $10-30 \mathrm{~ms}$ & $1-2 \mathrm{~ms}$ & $0-50 \mathrm{sccm}$ & $0-200 \mathrm{Gauss}$ & $150 \mathrm{kHz}, 1.6 \mathrm{kV}$ \\
\hline
\end{tabular}

\section{Figure 38. Summary of Magnetoshell operating and testing conditions}

Shown in Figure 39 is the time-resolved impulse measurements of jet and discharges of various lengths. By measuring the amplitude, period, and damping rate the impulse applied to the stand can be measured using standard techniques. With that impulse and an approximate 
A Plasma Aerocapture and Entry System for Manned Missions and Planetary Deep Space Orbiters

\section{MSNW LLC}

discharge time the average thrust can then be determined. Figure 40 is a summary of thrust results for the key cases of interest. These numbers have relatively large error bars, in that the estimate of average discharge and impingement length are quite uncertain. However, the three orders of magnitude increase in thrust is not questionable. The known cross sectional area of the Magnetoshell hardware is $20 \mathrm{~cm}^{2}$. By scaling the measured thrust with the known area, an equivalent Magnetoshell cross section can be determined. Therefore, the Magnetoshell measured an equivalent interception diameter of $1.6 \mathrm{~m}$ in a $2.2 \mathrm{~m}$ diameter chamber. This also approximately agrees with the photographic evidence. In conclusion, while the error in the absolute magnitude of thrust measurement may be quite high, the relative measurement and the large ratio between the bare and Magnetoshell cases is indisputable. An argon Magnetoshell was generated and using a supersonic jet, demonstrated concrete charge exchange, entrainment, and a $1000 \mathrm{X}$ increase in drag.

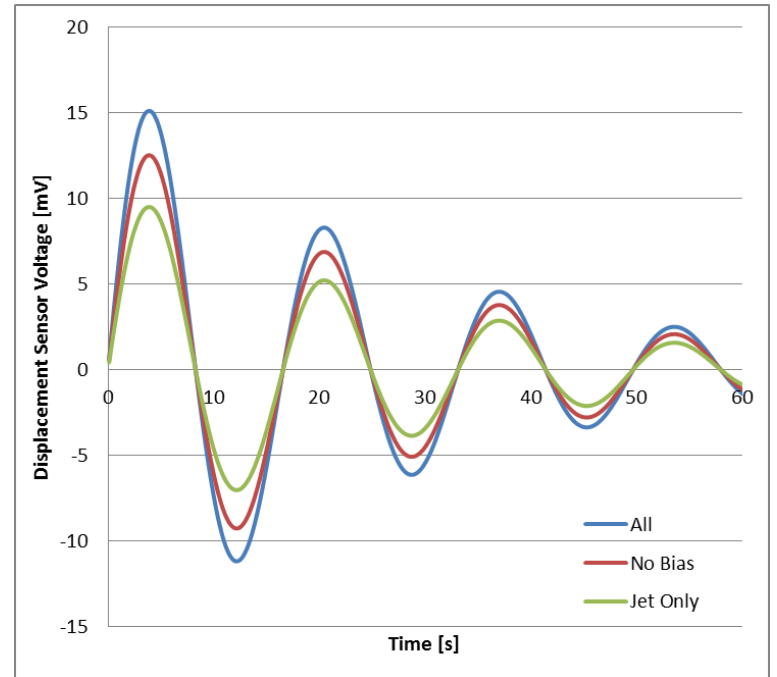

Figure 39. Magnetoshell impulse measurements for discharges of various lengths.

\begin{tabular}{|lccc|}
\hline Operating Condition & Measured Drag & Effective Area & Relative Drag \\
\hline Magnetoshell, MPD with Neutral Flow, with Bias & $220 \mathrm{mN}$ & $2.3 \mathrm{~m}^{2}$ & 1150 \\
\hline Magnetoshell, MPD with Neutral Flow, no Bias & $110 \mathrm{mN}$ & $1.1 \mathrm{~m}^{2}$ & 550 \\
\hline MPD with Neutral Flow and Bias & $190 \mu \mathrm{N}$ & $0.02 \mathrm{~m}^{2}$ & 1
\end{tabular}

Figure 40. Summary of Magnetoshell results, translating impulse into direct equivalent steady thrust measurements 
A Plasma Aerocapture and Entry System for Manned Missions and Planetary Deep Space Orbiters

MSNW LLC

\section{Orbital Modeling and General Mission Benefits}

\section{Mission Benefits and Architecture Consequences}

The first spacecraft to utilize upper atmospheric drag in a beneficial manner was HITEN, a Japanese spacecraft in 1991 that shed $1.7 \mathrm{~km} / \mathrm{s}$ of $\Delta \mathrm{v}$ from its highly elliptic orbit around Earth. [16] The Magellan Spacecraft was the first mission to use aerobraking on another planet. [17] Magellan used aerobraking maneuvers to lower it's apoapsis from about $8500 \mathrm{~km}$ to $540 \mathrm{~km}$, in order to conduct gravitational studies of Venus. This maneuver would have required $900 \mathrm{~kg}$ of propellant with traditional methods; however Magellan was able to accomplish this part of its mission with the less than the $100 \mathrm{~kg}$ left onboard. In 1997 the Mars Global Surveyor (MGS) also used aerobraking to shed $1200 \mathrm{~m} / \mathrm{s}$ of $\Delta \mathrm{v}$ to circularize its orbit after initial Mars orbit insertion [18]. For aerobraking, structure loads and thermal issues are the significant factor. For MGS the dynamic pressure was limited to $0.6 \mathrm{~N} / \mathrm{m}^{2}$ and the Magellan spacecraft experienced maximum dynamic pressure of $0.31 \mathrm{~N} / \mathrm{m}^{2}$. While small, these forces were mostly applied to their delicate cantilevered solar panels and communication equipment. With a Magnetoshell all the applied force will be through bending of magnetic field lines and transfer to the spacecraft through the antenna/magnets, which could easily accommodate much higher dynamic loads. The other limiting factor is thermal loading. With both previous aerobraking missions, drag was limited due to temperature constraints. For MGS this limit was $65^{\circ} \mathrm{C}$ and for Magellan it was $180{ }^{\circ} \mathrm{C}$. With a Magnetoshell the friction interacts with the plasma around the spacecraft and not the spacecraft itself, eliminating many, if not all, of the thermal considerations. It is important to note that in addition to the thermal, mass, and structural concerns the most important challenge with conventional aeroshells is the dramatic increase in risk as the braking force increases. The braking forces vary greatly with respect to inclination, time of year, time of day, solar cycle, solar phenomenon and even surface weather. Needless to say both aerobraking and aerocapture require in-depth knowledge and understanding of the atmosphere, and the ability to rapidly modify drag based on changing conditions making fixed surface aeroshells ill-suited for manned or rapid deceleration missions. Clearly, using aerobraking at very remote, poorly-understood planetary atmospheres, such as Jupiter or Neptune, is risky with current technology. The most widely studied aerobraking case, the MGS, had significant challenges with unanticipated thermal and structural loads. For the Plasma Magnetoshell AAES the drag force on the Magnetoshell is orders of magnitude larger than that on the spacecraft itself, so the drag force can be increased, or indeed turned-off, as needed by the craft.

Aerocapture has never been implemented to date, but there are numerous studies for it's application to Mars and other interplanetary missions [19]. These studies have illustrated the many advantages of using aerocapture. First, by reducing the amount of propellant needed at a destination planet, some missions, such as a Neptune Orbiter, become feasible by allowing the spacecraft to fit within the largest available launch vehicles. In addition, aerocapture can allow for faster orbital transfers, reducing trip time and radiation exposures. With faster trip times, higher approach velocities and thus increased delta- $\mathrm{V}$ requirements are encountered for capture. Figure 42 shows the delta- $\mathrm{V}$ requirements for missions to Mars and Jupiter as calculated by COPERNICUS. It can be seen that not only does delta- $\mathrm{V}$ increase with reduced trip time, but that the delta- $\mathrm{V}$ required to orbit the target planet is as large, if not even greater than the delta- $\mathrm{V}$ 
A Plasma Aerocapture and Entry System for Manned Missions and Planetary Deep Space Orbiters

MSNW LLC

required for the transfer orbit. By utilizing a low-mass Magnetoshell aerocapture, the delta-V requirements for a 1000-day all-propulsion Jupiter mission is the same as a 300 day with Magnetoshell braking. For Mars, the traditional 120-day trip can be reduced to less than half using conventional propulsion technologies!
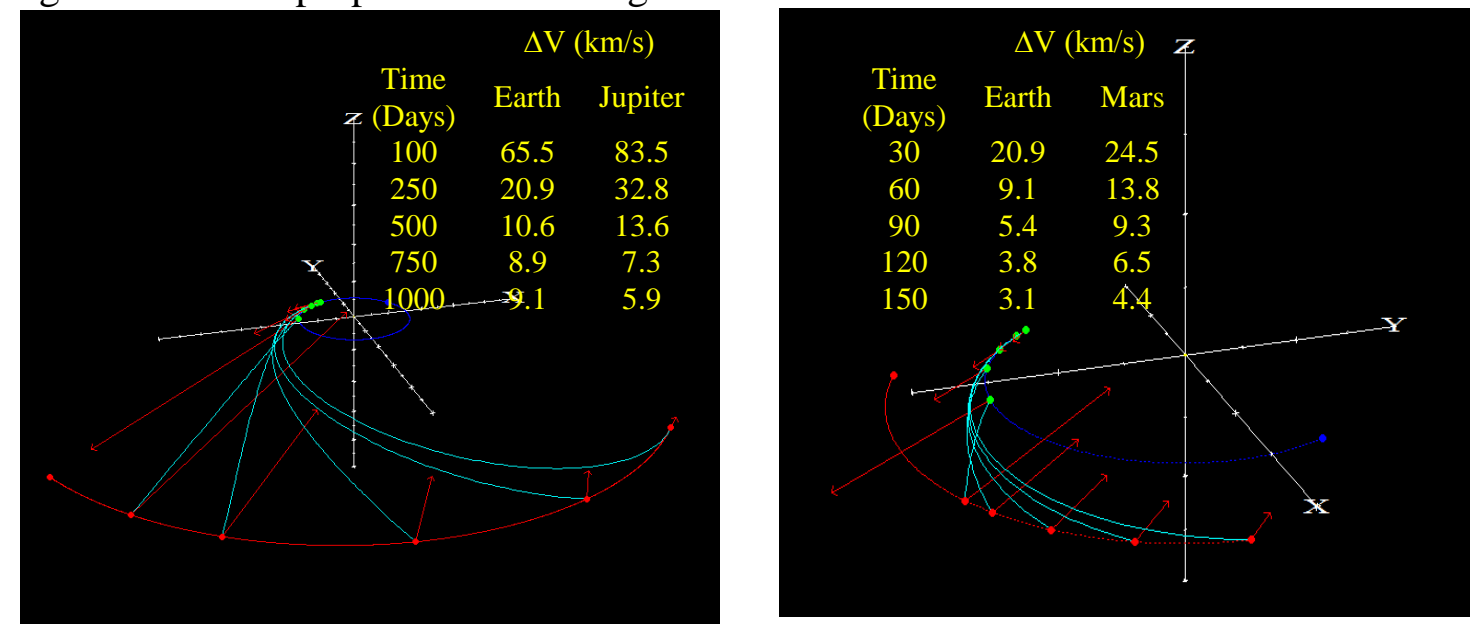

Figure 41. COPERNICUS simulations of impulsive orbital transfer from Earth (blue) to Jupiter and Mars (red), respectively. Shown are the delta-V requirements for Transfer and Insertion orbits. Delta-V requirements are given for the required location.

In addition to cargo missions, Plasma Magnetoshells would allow drastic design changes to crewed aspects of interplanetary missions architectures. DRA 5.0 does not us aerocapture for crew missions, largely because of the risk involved with conventional aeroshells. Plasma Magnetoshells allow for dynamic control at any altitude, therefore, they would be safe and reliable for human missions. Plasma Magnetoshells would add additional safety and health benefits as the plasma Magnetoshell could also be deployed during Mars transit to eliminate the effects of solar flares should they arise. It would have little effect on GCR as shown by numerous previous analyses, however [20]. Stopping GCR is a formidable challenge and the most effective solution is simply to reduce exposure. Plasma Magnetoshells would allow for faster transit times between planets thereby reducing exposure. Figure 42 shows general mission parameters for the three mission of high interest.

$\begin{array}{lccc}\text { Mission } & \text { Mars Manned } & \text { Venus Sample Return } & \text { Neptune Orbiter } \\ \text { Incoming Velocity } & 5.5 \mathrm{~km} / \mathrm{s} & 10.6 \mathrm{~km} / \mathrm{s} & 26.7 \mathrm{~km} / \mathrm{s} \\ \text { Neutral Molecular Weight } & 43.4 \mathrm{amu} & 43.4 \mathrm{amu} & 2.5 \mathrm{amu} \\ \text { Ion Average Weight } & 15.0 \mathrm{amu} & 14.6 \mathrm{amu} & 1.6 \mathrm{amu} \\ \text { Directed Neutral Energy } & 7.0 \mathrm{eV} & 25.5 \mathrm{eV} & 9.4 \mathrm{eV} \\ \text { Aeroshell Neutral Density } & 1 \mathrm{E}-6 \mathrm{~kg} / \mathrm{m}^{3} & 2.2 \mathrm{E}-7 \mathrm{~kg} / \mathrm{m}^{3} & 1.5 \mathrm{E}-8 \mathrm{~kg} / \mathrm{m}^{3} \\ \text { Aeroshell Particle Density } & 1.3 \mathrm{E} 19 \mathrm{H}^{3} & 3 \mathrm{E} 18 \mathrm{\#} / \mathrm{m}^{3} & 3.5 \mathrm{E} 18 \mathrm{\#} / \mathrm{m}^{3} \\ \text { Figure 42. Comparison of mission requirements for Aerocapture. } & \end{array}$


A Plasma Aerocapture and Entry System for Manned Missions and Planetary Deep Space Orbiters

\section{MSNW LLC}

\section{Neptune Orbiter Mission}

The primary mission investigated in Phase I is a Neptune Orbiter with Probes Mission. This is based off of the Neptune Orbiter Mission designed by NASA JPL. This mission had several iterations and designs but fundamentally was to launch a Cassini type mission to Neptune. This would include one 800-kilogram science orbiter and two 100-kilogram probes to land on Triton. The key mission goals are below.

- Study the interior structure, composition, and atmospheric dynamics of an "ice giant," with probes to 100 bars and orbiter remote sensing over 3-year orbital tour

- Investigate the surface, interior, and atmosphere of Triton, which may be a captured Kuiper Belt Object, with $>40$ flybys (altitude $<1000 \mathrm{~km}$ ) and possibly a Triton lander

- Observe the response of the magnetosphere to daily "pole on" orientation to the solar wind, with 3-year orbital tour

- Study Neptune's unique ring arcs and associated moons over 3 year mission, with 3-year orbital tour

Figure 43 showed an artist's rendition of the Neptune Orbiter craft and below are the critical mission parameters. It is important to notice this required a five-meter diameter faring and had on board only 212 Watts of RPG power. This mission was investigated with both a traditional propulsive deceleration which limited the available science payload with a Delta IV heavy launch to only 180 kilograms. This mission was revisited in 2005 with an aerobraking and aerocapture component. This provided an $800 \%$ increase in delivered payload to Neptune and was an enabling technology for the mission. A current aerocapture system design was completed by NASA and is shown in Figure 44. This preliminary design, while enabling and critical to allowing a Neptune orbiter mission, still has significant technical challenges and several significant technology development efforts would be required to develop aeroshell-based aerocapture to sufficient technical readiness.

Therefore, this is a perfect candidate for investigating the use of a Magnetoshell-based

surveys as well as one of the highest mission priorities for interplanetary robotic science. The primary mission goals are to investigate the unique magnetic and atmospheric structure of Neptune as well as possible liquid water on Triton and several moons that may be captured Oort cloud objects.

- $800 \mathrm{~kg}$ science payload

- $229 \mathrm{~kg}$ deployable probes

- $885 \mathrm{~kg}$ Aeroshell and TPS

- $3730 \mathrm{~kg}$ LEO Mass

- $5 \mathrm{~m}$ fairing

- 212 W MMRTG 
A Plasma Aerocapture and Entry System for Manned Missions and Planetary Deep Space Orbiters

MSNW LLC
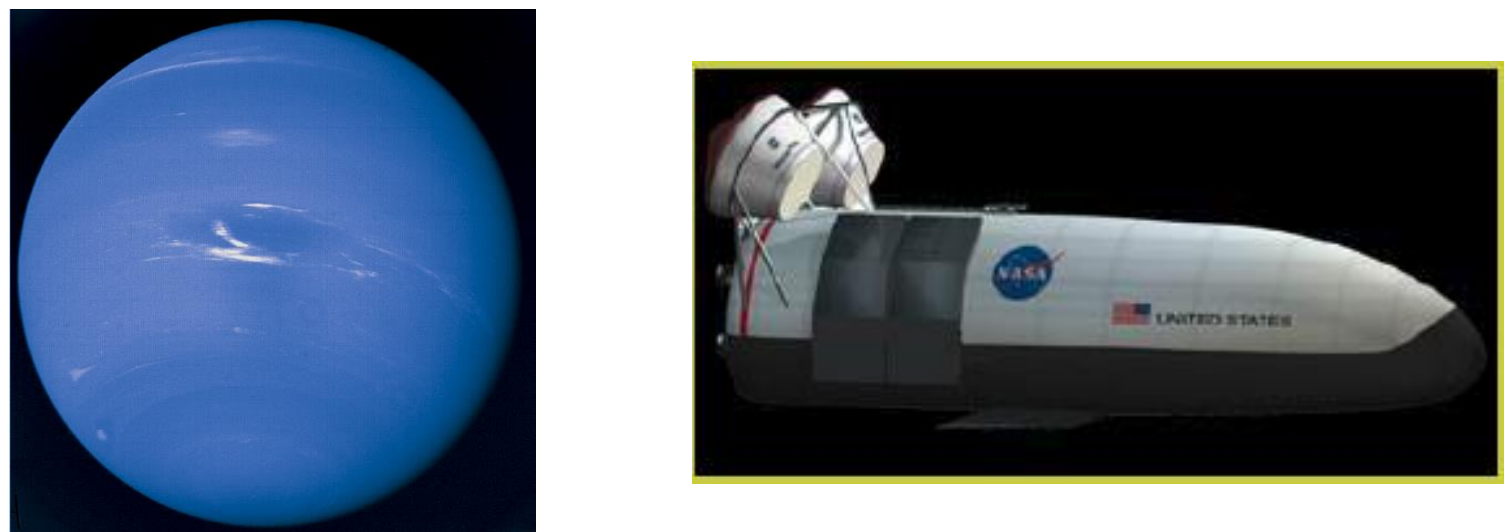

Figure 43. Photograph of Neptune and an artist's rendition of the Neptune Orbiter [21].
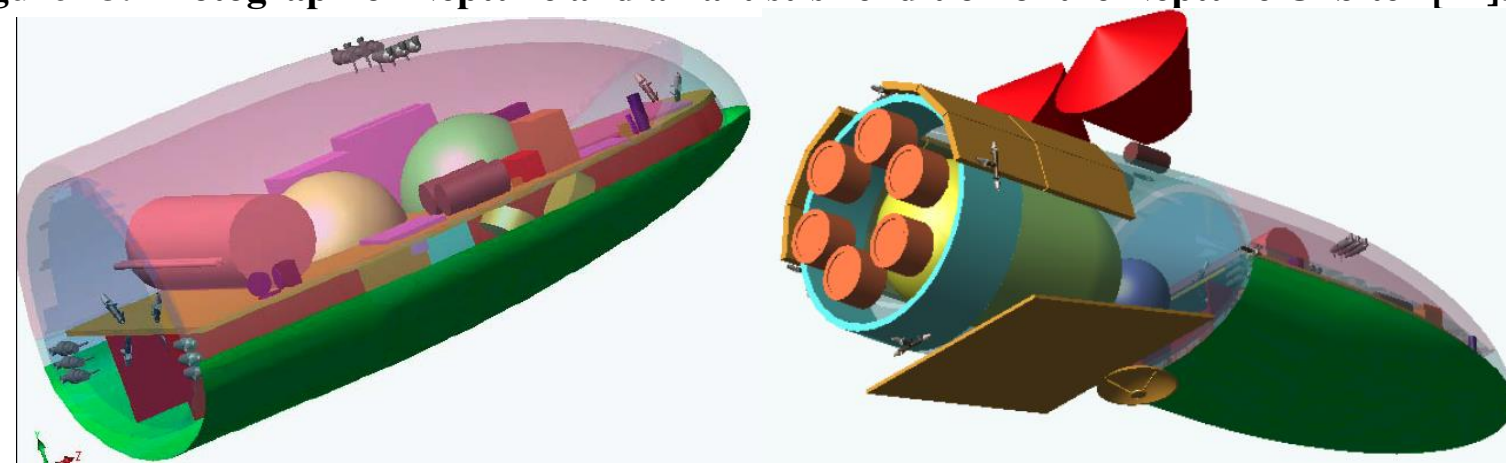

Figure 44. Engineering drawing of the Neptune Orbiter and heatshield. 
A Plasma Aerocapture and Entry System for Manned Missions and Planetary Deep Space Orbiters

MSNW LLC

\section{Magnetoshell Optimization and Design for Neptune Mission}

A systems level optimization of the Magnetoshell hardware was completed. Equations 15 through 22 detail a primary design metric and will now be briefly described. The complete list of variables are provided in the nomenclature section. Equation 15 shows the full equation for a finite solenoidal magnetic field solution on axis. This is a strong function of current, I, and coil turns, $\mathrm{N}$, as well as radius, $\mathrm{R}_{0}$. It is a weak function of coil length, 1 , and thickness, $t$. Equation 16 shows the magnetic dipole falloff for a low beta magnetic field configuration. This is a cubic radial falloff that is a very strong function of initial coil radius. The collection radius, $\mathrm{R}_{\text {dipole }}$ of the Magnetoshell as described earlier in the transient and steady plasma model can be approximated at simply the outer limit of the magnetic gyro radius, $\lambda_{i}$ which is the largest distance at which a magnetized and trapped charge exchange particle can be contained long enough to exchange momentum to the spacecraft. This can be integrated into Equation 17 and solved simply as a function of the initial magnetic field, $\mathrm{B}_{0}$ and flow parameters. At this point, the physical characteristics of a given magnetic coil can be investigated with simple straightforward relationships of an aluminum metallic finite coil. Equation 18 shows the power required to operate this coil. Equation 19 shows the mass of the coil itself. Equation 20 is the mass of a battery system powering the coil. It is assumed a specific power, $\alpha_{b}$ for the battery mass measured in kilograms per kilowatt and a specific burn time and/or operation time, $\Delta \mathrm{t}_{\mathrm{b}}$ in which the Magnetoshell is active. The required power system to recharge that battery is shown in Equation 21 again assuming a steady specific power, $\alpha_{b}$, and a coast period, $\Delta t_{c}$ in which the battery is being recharged. Finally, Equation 22 shows the peak temperature of the magnet. In this case, Equation 22 includes the specific heat, $\mathrm{C}_{\mathrm{p}}$, mass of the coil, and burn time as well as balancing input electrical power and output radiation rates.

$$
\begin{gathered}
B_{0}=\frac{\mu_{0} I N}{2 t} \ln \left[\frac{\sqrt{\left(R_{0}+t\right)^{2}+(l / 2)^{2}}+\left(R_{0}+t\right)}{\sqrt{\left(R_{0}-t\right)^{2}+(l / 2)^{2}}+\left(R_{0}-t\right)}\right] \approx \frac{\mu_{0} I N}{R_{0}} \\
B=B_{0}\left(\frac{R}{R_{0}}\right)^{3} \approx \frac{\mu_{0} I N}{R_{0}}\left(\frac{R_{0}}{R}\right)^{3} \\
R_{\text {dipole }} \approx \frac{\lambda_{i}}{\pi} \approx \frac{\sqrt{m_{i} T_{i-e V}}}{B \pi 10^{4}} \approx \sqrt{\frac{B_{0} \pi 10^{4} R_{0}^{3}}{\sqrt{m_{i} T_{i-e V}}}} \\
P_{e}=I^{2}\left(N^{2} \frac{2 \pi \rho_{c} R_{0}}{t l}\right) \\
m_{c}=4 \pi \rho_{m} R_{0} l t \\
m_{B}=\alpha_{B} P_{e} \Delta t_{B} \\
m_{P}=\alpha_{P} P \frac{e^{\Delta} \Delta t_{B}}{\Delta t_{C}}
\end{gathered}
$$


A Plasma Aerocapture and Entry System for Manned Missions and Planetary Deep Space MSNW LLC

$$
T_{c-n+1}=\frac{C_{p}}{m_{c}}\left(\left(P_{e}-\sigma\left(T_{c-n}^{4}-T_{\infty}^{4}\right)(2 \pi r(2 t+l))\right)\right) \Delta t_{B}
$$

For these solutions, the burn times were quite short and the radiated power was a minor contributor and could be ignored. In figure three, typical scaling for electrical power and magnet mass are shown. Dotted lines show magnet mass for two diameters and two widths while solid lines show power requirement for a 250 Gauss magnet.

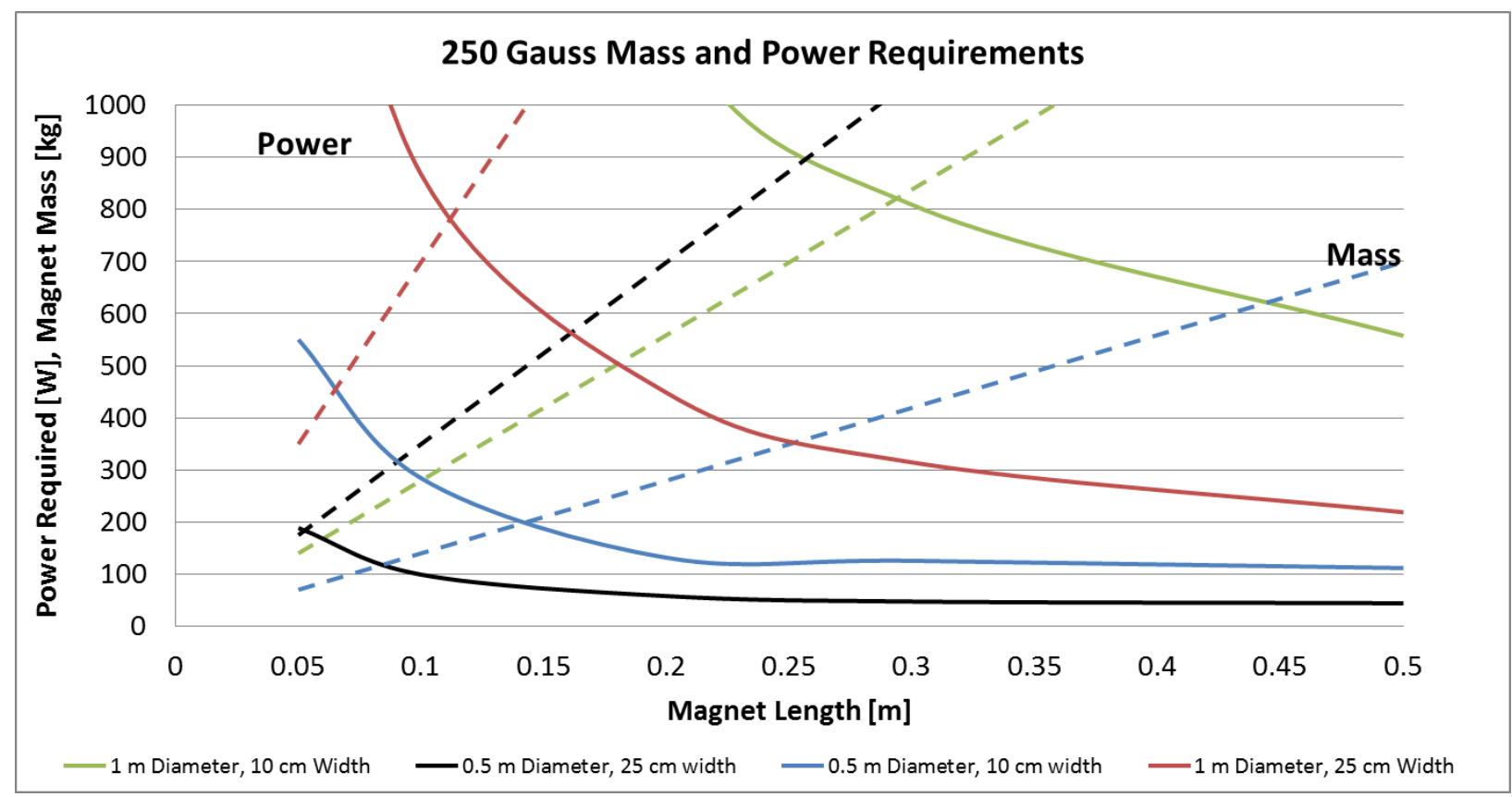

Figure 45. 250 Gauss magnet mass and size. Shown is copper and steady heating.

The same analysis was performed with a simple cylindrical rod as an approximation of Figure 46 
A Plasma Aerocapture and Entry System for Manned Missions and Planetary Deep Space Orbiters

MSNW LLC

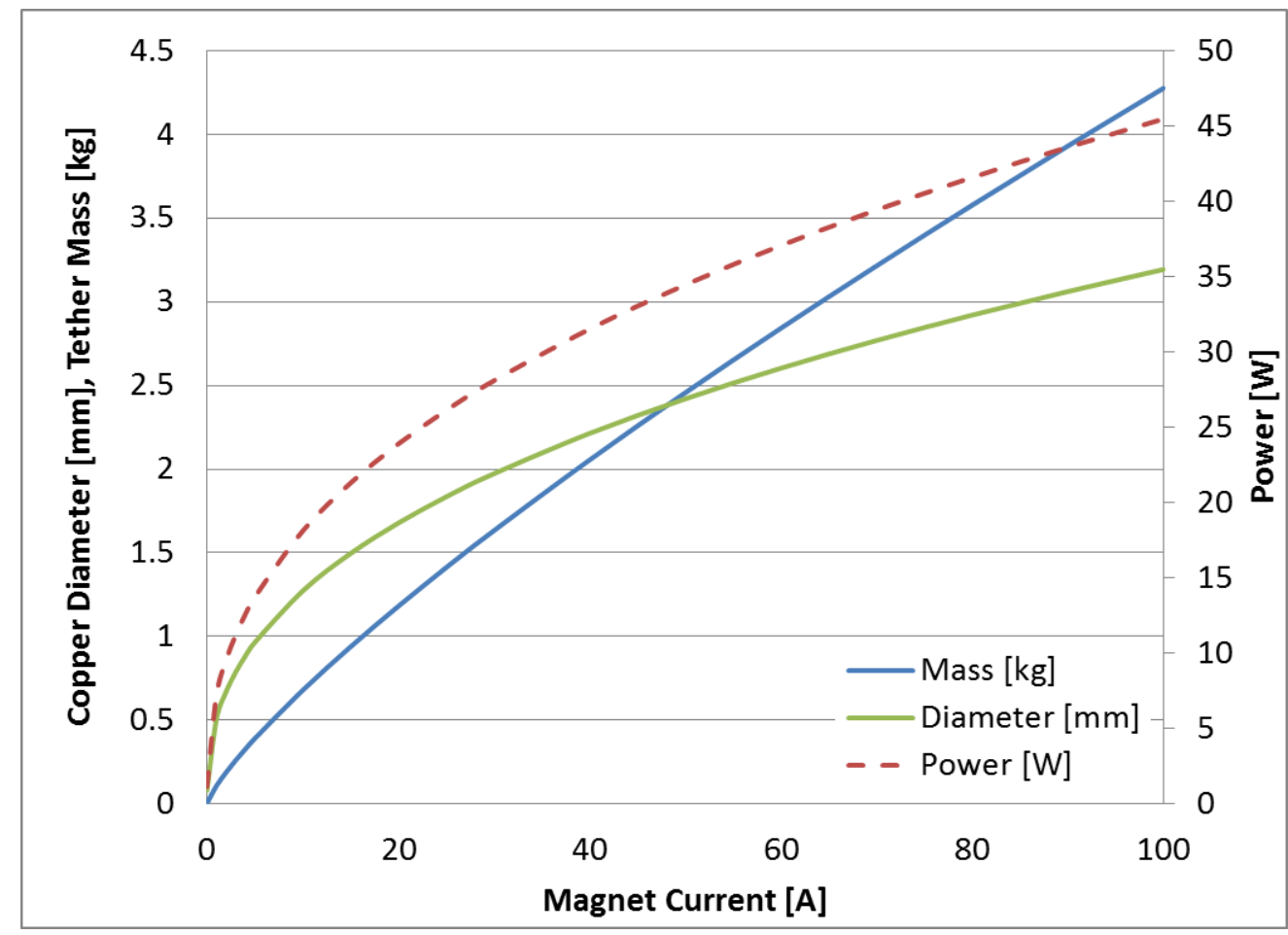

Figure 46. Tether mass, size, and power requirements of a copper tether. Shown is copper and steady heating.

Magnet temperature and power was a difficult optimization challenge. To fill a many meter dipole with magnetic energy and sustain that energy presents a unique design challenge. It was found very early in the design process that the required instantaneous power to sustain a Magnetoshell was greater than $10 \mathrm{~kW}$, for the large scale manned missions greater than $100 \mathrm{~kW}$. That is significantly above the available power, particularly for deep space missions. However, just as a traditional Aeroshell does not have to sustain steady thermal loads, a Magnetoshell does not need steady power. Therefore, very quickly a battery power system becomes optimal in which a low power system (as in the $200 \mathrm{~W}$ MMRTG on the Neptune Orbiter) charges the batteries which only discharge during the entry phase of the mission. Therefore, energy requirements and thermal requirements must sustain the magnet coil, PPU, and tether but not a steady shell. This then requires knowledge of the orbital trajectory which will be described in the following section.

Due to the sheer number of optimization parameters, a Monte Carlo approach was taken in which a random sampling of possible magnet geometry and operational conditions were simulated by running this simulation for extended periods and down-selecting to the most promising candidates that are still geometrically possible. The optimum magnet design for a desired braking diameter and force could be then determined. In Figure 47 and Figure 48, two model solutions are shown with a wide range of input parameters. Magnet radius was allowed to vary between zero and two meters; temperature to 800 Celsius. The power system includes a 200-watt MMRTG [22] and a battery specific mass of $1 \mathrm{MJ} / \mathrm{kg}$ [23]. The solution set shown does not include the less-optimal candidates which are much higher system mass but lower dipole 
A Plasma Aerocapture and Entry System for Manned Missions and Planetary Deep Space Orbiters

\section{MSNW LLC}

radius i.e. five-meter coil that weighed 300 kilograms was not competitive and not included in the graph. Figure 47 is unlimited peak power from the battery while Figure 48 is limited to peak power output of $10 \mathrm{~kW}$ from the battery. It can be seen then that a wide range of effective magnetic dipoles is possible for this mission from $5 \mathrm{~m}$ to $14 \mathrm{~m}$ in diameter. A $9 \mathrm{~m}$ dipole and 159 $\mathrm{kg}$ Magnetoshell and power system was chosen as the optimal based off of orbital modeling results that will be presented later. For a two-meter magnet, Figure 49 Figure 50, and Figure 52 show system mass scaling as a function of a variety of operational parameters. Figure Figure 49 shows the total power system mass as a function of total Magnetoshell mass optimizing at a 72kilogram battery and RTG system. Figure 50 shows magnet mass as a function of magnet length, and it can be seen that this is a very strong function as both field and mass scale with length. Figure 51 shows operating temperature as a function of total system mass. Interestingly, this was also a strong function but in an optimal way. By increasing the system mass ten kilograms, the magnet temperature could be decreased almost 500 degrees. A summary of the critical parameters are shown in Figure 52 for a Neptune mission. The trailing tether was also optimized assuming the batteries were on board the spacecraft. The tether was designed such that it was capable at radiating any power dissipated in the umbilical and in general was a minor mass contribution. Figure 46 shows the design of the umbilical and an optimal 30-meter long 4.6kilogram tether was chosen.

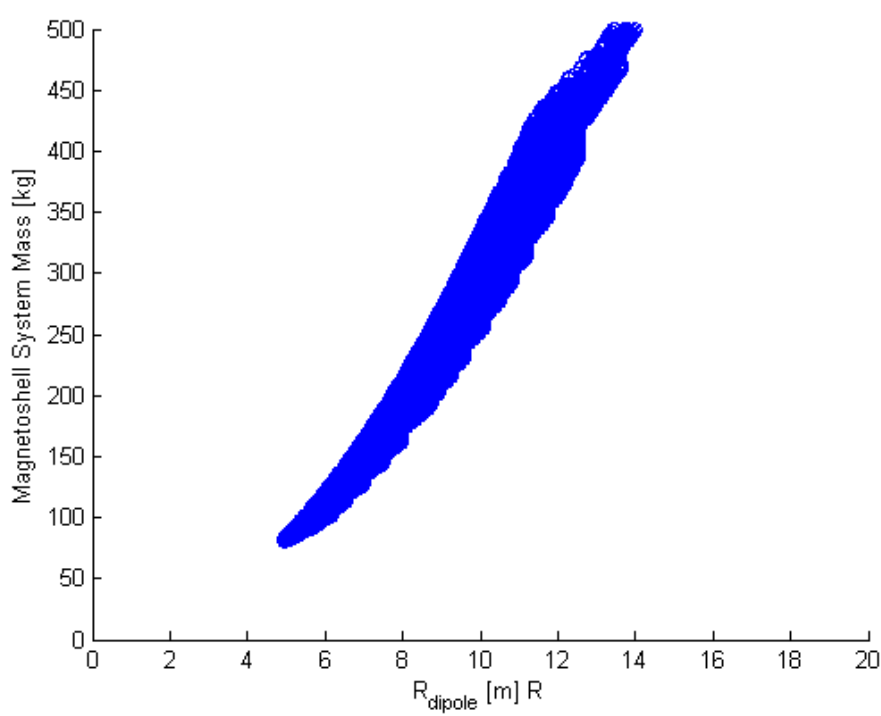

Figure 47. Model solutions for a Neptune orbit insertion. Shown are all permutations for a wide range of magnet sizes and power systems. Battery backed and temperature limited. 
A Plasma Aerocapture and Entry System for Manned Missions and Planetary Deep Space Orbiters

MSNW LLC

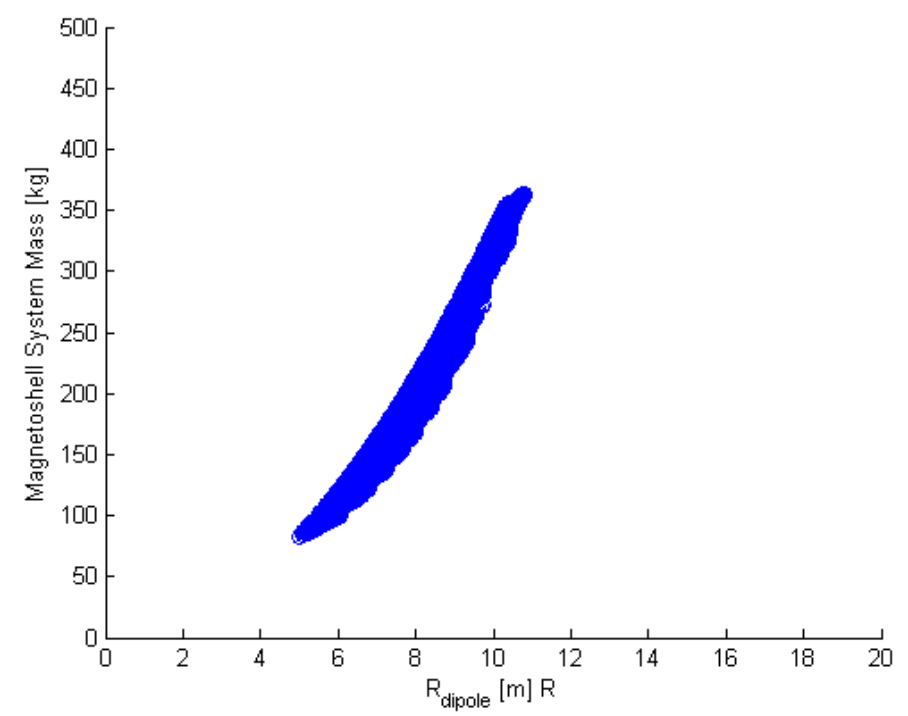

Figure 48. Model solutions for a Neptune orbit insertion limiting peak power to $10 \mathrm{~kW}$ (from the battery). Shown are all permutations for a wide range of magnet sizes and power systems. Battery backed and temperature limited.

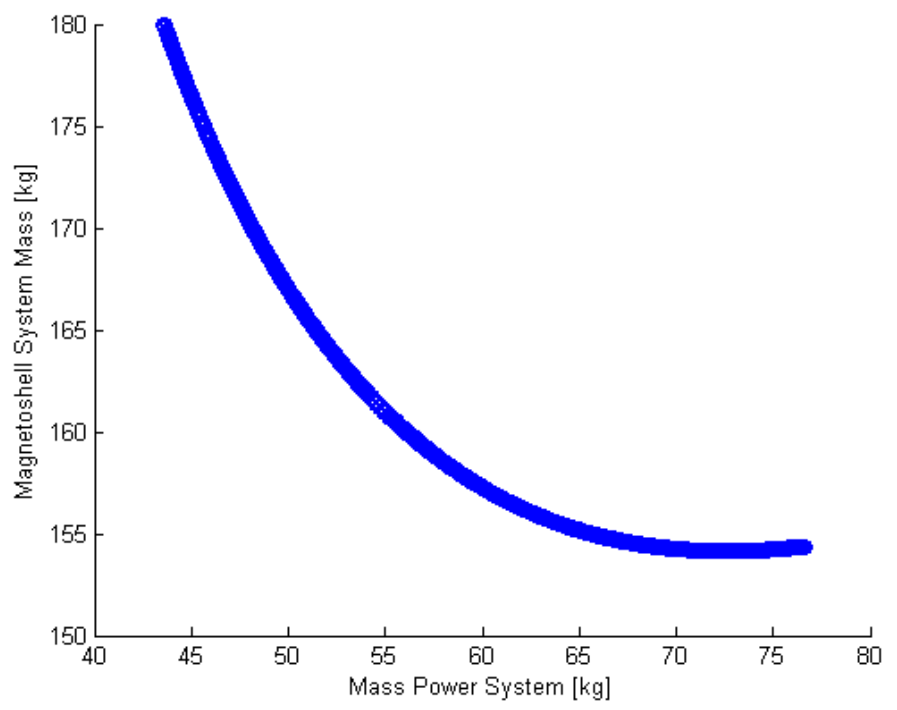

Figure 49. Model solutions for power system (battery, RTG) versus total system mass for a Neptune orbit insertion. Shown is a fixed $2 \mathrm{~m}$ radius magnet. 
A Plasma Aerocapture and Entry System for Manned Missions and Planetary Deep Space Orbiters

MSNW LLC

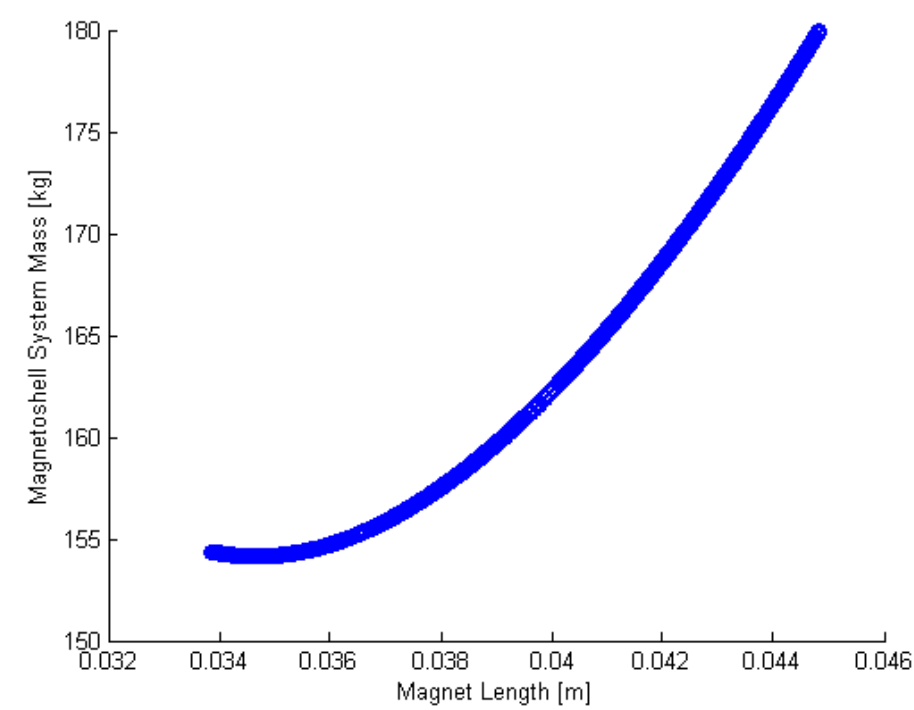

Figure 50. Model solutions for magnet length and thickness versus total system mass for a Neptune orbit insertion. Shown is a fixed $2 \mathrm{~m}$ radius magnet.

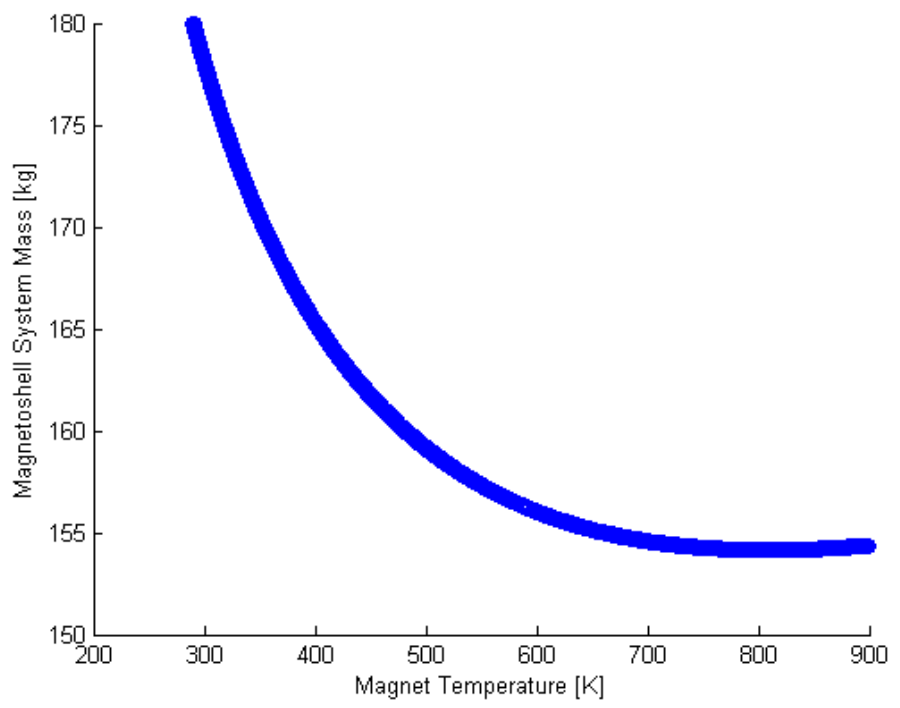

Figure 51. Model solutions for peak operating temperature versus total system mass for a Neptune orbit insertion. Shown is a fixed $2 \mathrm{~m}$ radius magnet. Clear minimum system mass for high temperature, but small increases in mass dramatically lower peak temperature. 
A Plasma Aerocapture and Entry System for Manned Missions and Planetary Deep Space

Orbiters

MSNW LLC

\begin{tabular}{l|l} 
Neptune Magnetoshell Design Parameter & Optimum Configuration \\
\hline Magnet Average Radius & 2 Meter Aluminum \\
Total Magnet Weight & $81 \mathrm{~kg}$ \\
Total Battery and Solar Power Weight & $72 \mathrm{~kg}$ \\
Magnet Length & $3.5 \mathrm{~cm}$ \\
Magnet Thickness & $3.5 \mathrm{~cm}$ \\
Number of Turns & 4000 \\
Tether Geometry & 30 meters long, $4.6 \mathrm{~kg}$ \\
Peak Current & 9 Amps \\
Effective Aerobraking Radius & 9 Meters \\
Total Weight with 30\% growth & $207 \mathrm{~kg}$ \\
Equivalent Aeroshell Weight & $885 \mathrm{~kg}$
\end{tabular}

Figure 52. Summary of Magnetoshell parameters for a Neptune Orbiter insertion.

\section{Neptune Transfer with Insertion}

The NASA code Copernicus was then used to simulate a Neptune aerocapture mission. This uses a $1000 \mathrm{~kg}$ spacecraft and an effective dipole and drag radius of 9 meters. An impulsive burn was performed at earth to get on a direct Neptune injection orbit. This orbit then intersected approximately $900 \mathrm{~km}$ above Neptune and using the atmospheric model given in Figure 3, drag and thus aerocapture was modeled. In Figure 53, the orbital transfer is shown and in Figure 54, the simulation of aerobraking can be seen. For this calculation, a large initial dipole diameter is required to provide the initial orbital capture. This 9-meter dipole is operated for approximately 430 seconds. For the secondary orbits, the dipole diameter is reduced to only 4 meters for a slower aerobraking. Without this decrease the drag surface area is too large and a propellantheavy burn would have to be made to prevent full atmospheric entry. During the peak density, Figure 55 through Figure 60 show the resulting calculations for the behavior of the Neptune orbiter, aerocapture, and aerobraking. For this mission, six orbits are required over a two-day deceleration period. The initial Neptune relative velocity is about $5 \mathrm{~km} / \mathrm{s}$, which dramatically increases near apogee. During the first pass and initial capture braking force of $150 \mathrm{~N}$ is generated. A peak dynamic pressure of $0.3 \mathrm{~N} / \mathrm{m}^{2}$ is applied to the shell and spacecraft body. This is well within the limit of an orbiter system and several orders of magnitude lower than a traditional aeroshell space braking system. For this model, simple orbital dynamics is used while in a complete mission design in phase two likely would include a Jupiter orbital assist and a solar electric spiral stage at Neptune. 
A Plasma Aerocapture and Entry System for Manned Missions and Planetary Deep Space Orbiters

MSNW LLC

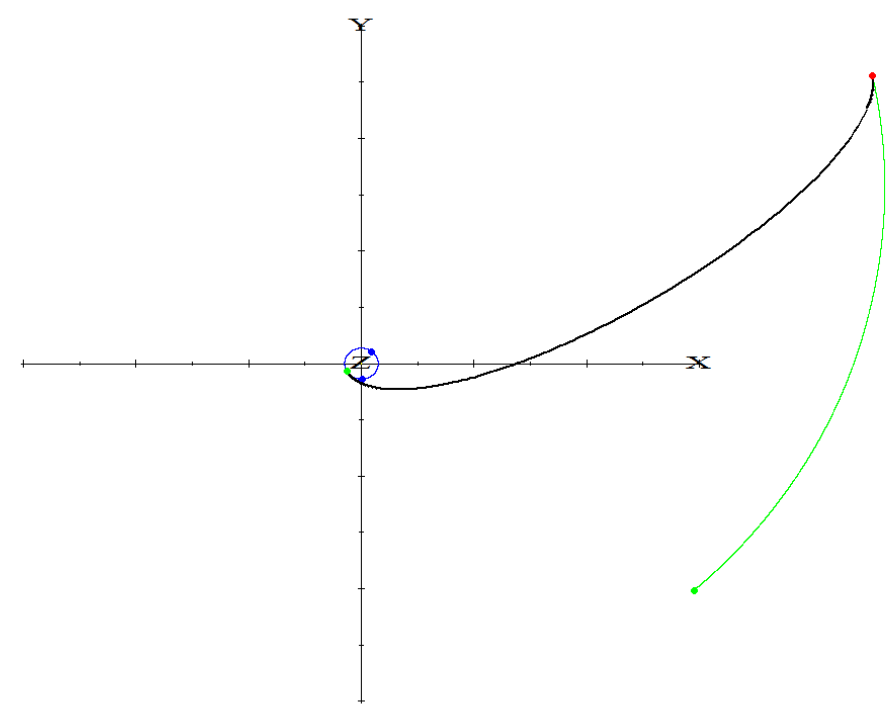

Figure 53. COPERNICUS simulations of impulsive orbital transfer from Earth (blue) to Neptune in a Heliocentric frame.

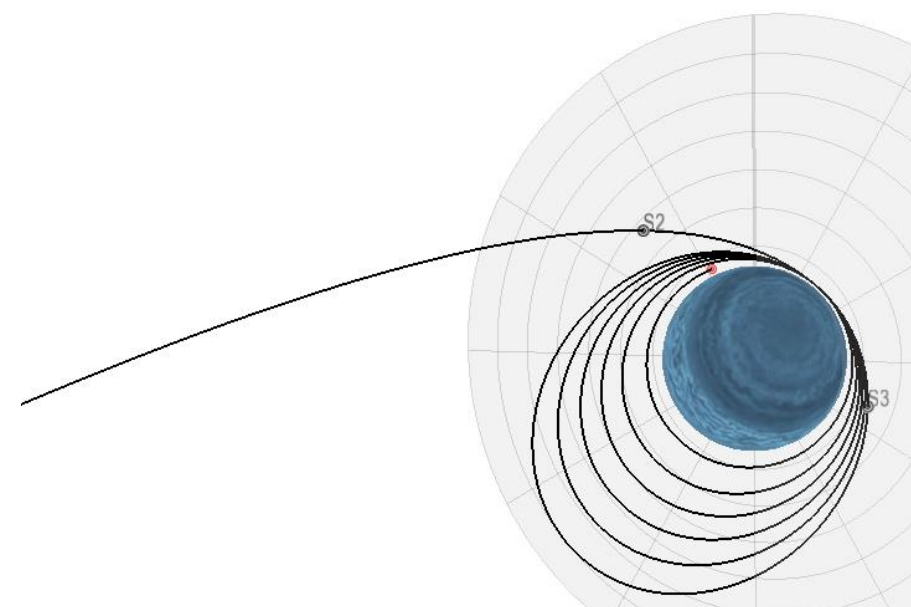

Figure 54. COPERNICUS simulation of atmospheric braking. 
A Plasma Aerocapture and Entry System for Manned Missions and Planetary Deep Space Orbiters

MSNW LLC

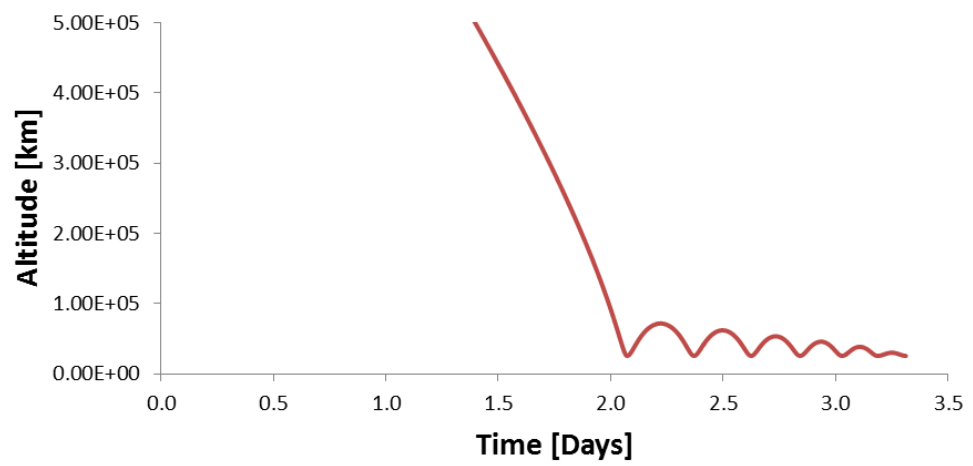

Figure 55. Neptune Aerocapture altitude.

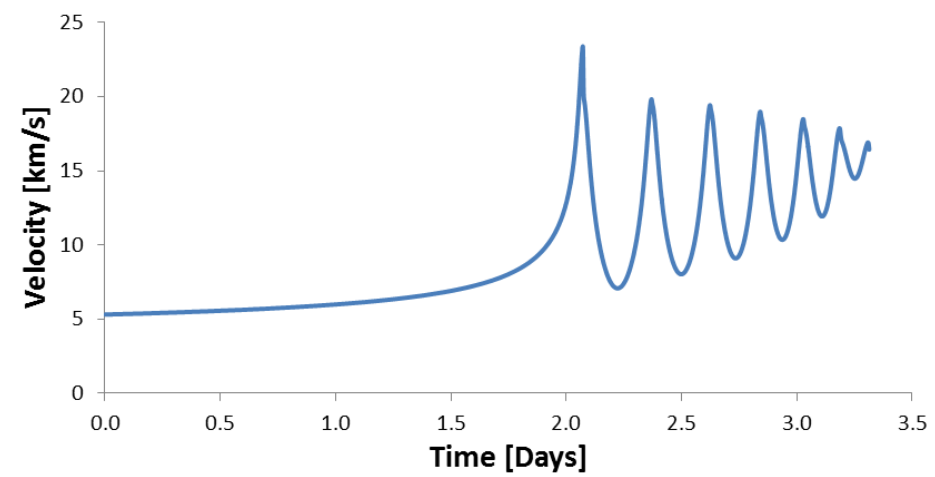

Figure 56. Neptune aerocapture velocity.

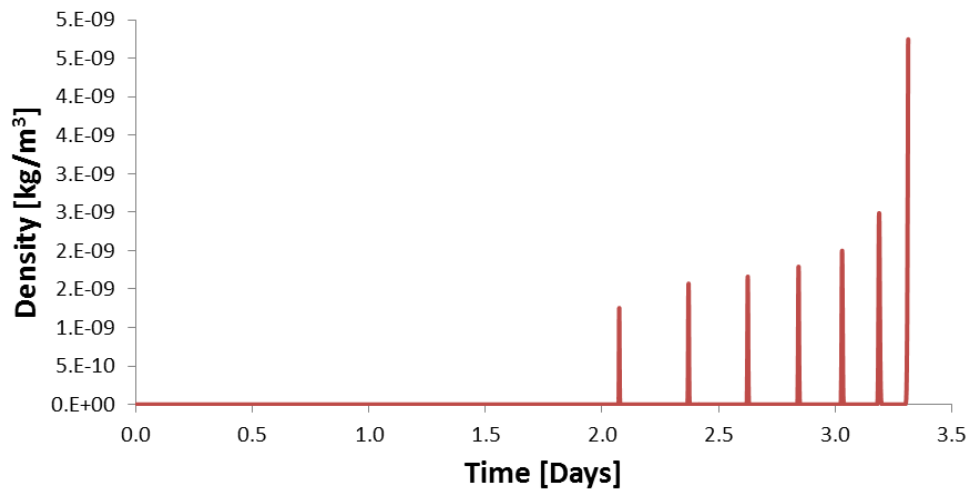

Figure 57. Neptune aerocapture atmospheric density. 
A Plasma Aerocapture and Entry System for Manned Missions and Planetary Deep Space Orbiters

MSNW LLC

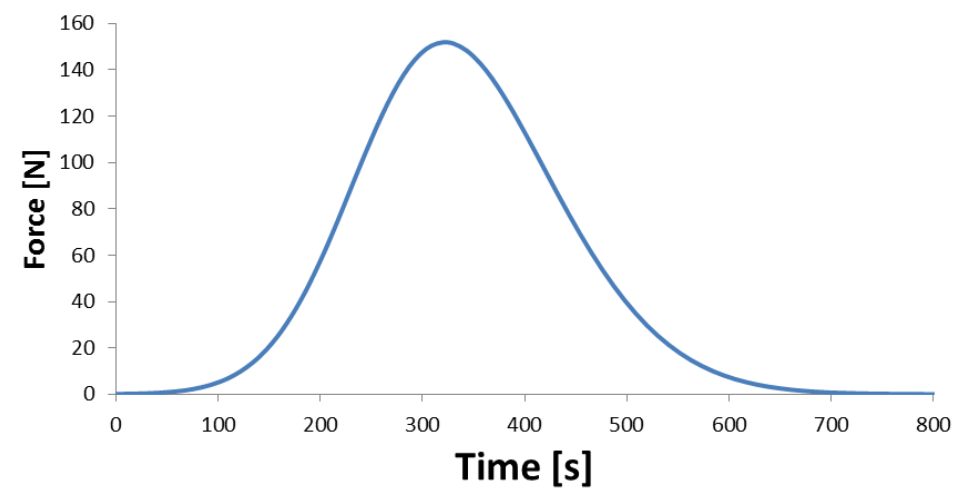

Figure 58. Close up of the Neptune drag force for the initial insertion.

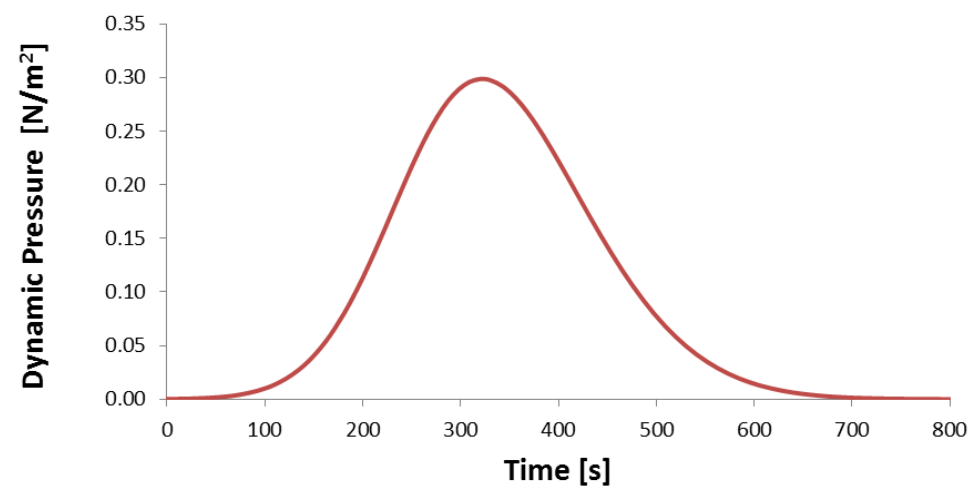

Figure 59. Close up of the Neptune dynamic pressure for the initial insertion.

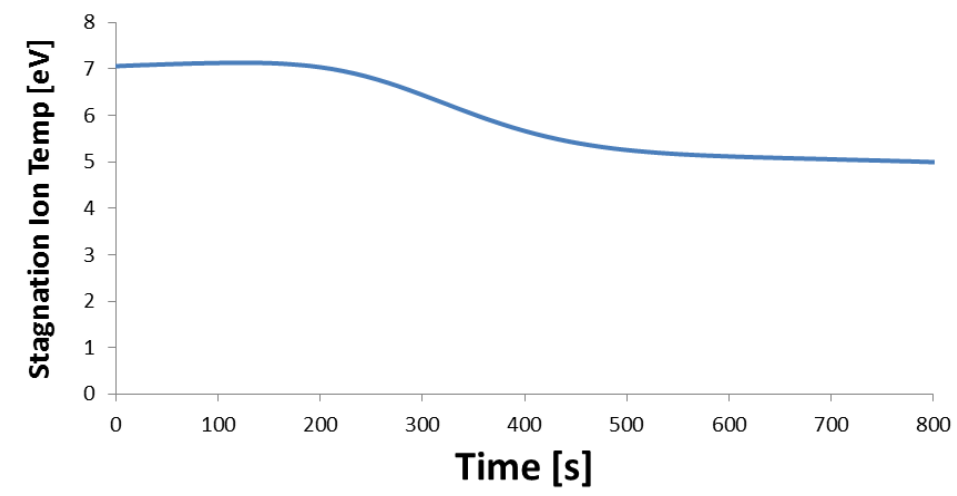

Figure 60. Incoming equivalent ion temperatures. 
A Plasma Aerocapture and Entry System for Manned Missions and Planetary Deep Space Orbiters

MSNW LLC

\section{Mars Orbital Modeling}

Using the Magnetoshell system model developed in the previous section, a 60 metric ton large-scale Mars aerocapture mission similar to DRA 5.0 was simulated. This mission is significantly more ambitious than the Neptune mission in that the aerocapture must shed the kinetic energy of a 60 metric ton payload. DRA 5.0 [24] has shown that for the higher acceptable risk Martian cargo missions, aerocapture using $20 \mathrm{MT}$ aeroshells can reduce the launch mass and cost of the mission dramatically. DRA 5.0 currently requires a minimum of 848.7 MT IMLEO. This mass is assembled into two cargo vehicles and one crewed spacecraft headed to Mars. This design architecture uses Nuclear Thermal Propulsion (NTP) for Trans-Mars Injection and aerocapture (for the cargo) for Mars Orbital Insertion (MOI). The $1000 \mathrm{~kg}$ Plasma Magnetoshell eliminates the need for massive heat shields, reducing the require mass by 80 MT. Additionally, 46 tons of propellant and 14 tons of tankage are saved at MTI due to the lighter payload masses. For the all propulsion crewed mission, $84 \mathrm{MT}$ of propellant and tanks are not needed for MOI with a low-risk Magnetoshell. In total the Magnetoshell Aerocapture has the capability to save 224 MT at IMLEO or on the order of \$2 Billion in launch costs as well as decrease risk. DRA 5.0 also outlines a mission architecture using chemical propulsion instead of NTP. For this architecture the Plasma Magnetoshell would result in even larger mass savings of 355 MT. NASA Design Reference architecture DRA 5.0 focuses mainly on the launch vehicle, interplanetary transportation and entry, decent and landing (EDL). The Plasma Magnetoshell is designed mainly as interplanetary transportation subsystem, resulting in huge saving at IMLEO. While it could be used for EDL, it is not the primary goal of this initial investigation. Any benefits with regards to EDL, would be small compared to the mass savings of the MOI and will be considered in the Phase II effort.

To provide Aerocapture for a manned Mars class mission requires then a significantly larger dipole operating at higher power with only minor increases in available geometric scale, compared to Neptune. Figure 61 shows the complete set of model solutions for a Mars orbit insertion of a 60 metric ton payload from an earth to orbit insertion. This shows that a Magnetoshell from 5 to 40 meters in radius is possible with large physical magnets. Assuming a magnet that can fit in a typical faring size requiring no expansion or mechanical processes, the solution set is shown in Figure 62 and is both heavier and more limited. However, for this mission, the simpler engineering option was chosen and a 2.5 -meter diameter magnet was selected. Using this 2.5-meter diameter magnet and a peak magnetic field of 100 Gauss, a 21meter dipole can be generated with peak pressure of up to $130 \mathrm{~N} / \mathrm{m}^{2}$. The pressure is significantly higher than what can be supported by a traditional spacecraft system, therefore some aeroshell must be retained. The solution set for this magnet is shown in Figure 63 with an optimal 275kilogram battery system. Phase II mission studies will seek to clarify these details. The solar power system was not included in this mission architecture. In Figure 64, system mass as a function of magnet length is shown. In this case, thickness was also allowed to vary and shows a significant effect on overall system mass. The magnet length was limited to 0.5 meters out of structural considerations for the magnet design. Finally, temperature is shown as in the Neptune mission to be a weak function of overall system mass. The complete magnet design is shown in Figure 66 
A Plasma Aerocapture and Entry System for Manned Missions and Planetary Deep Space Orbiters

MSNW LLC

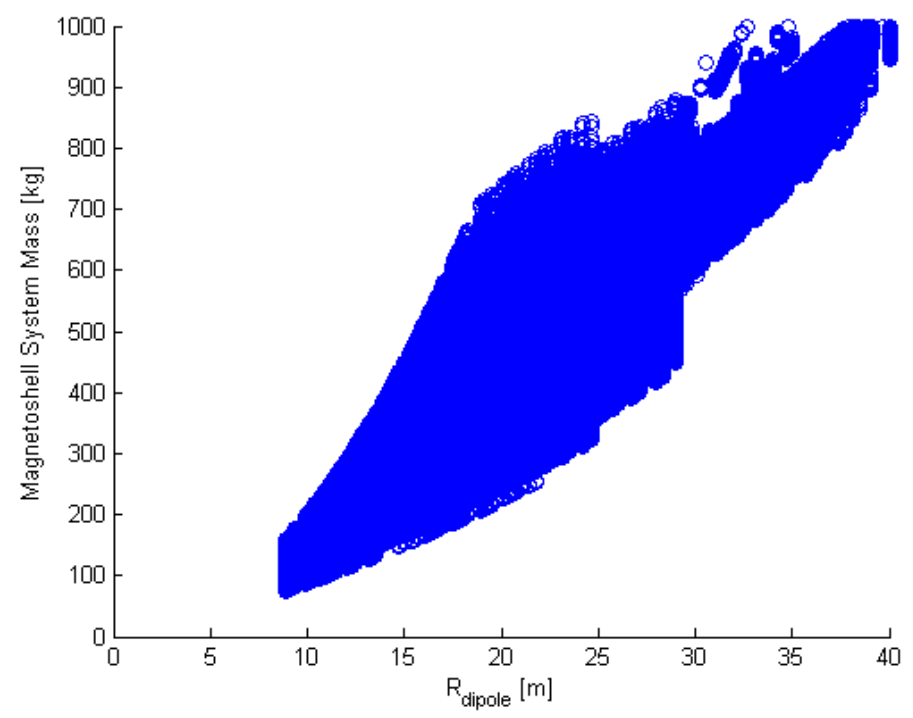

Figure 61. Model solutions for a Mars orbit insertion for a large magnet coil. Shown are all permutations for a wide range of magnet sizes (up to 5 meters) and power systems. Battery backed and temperature limited.

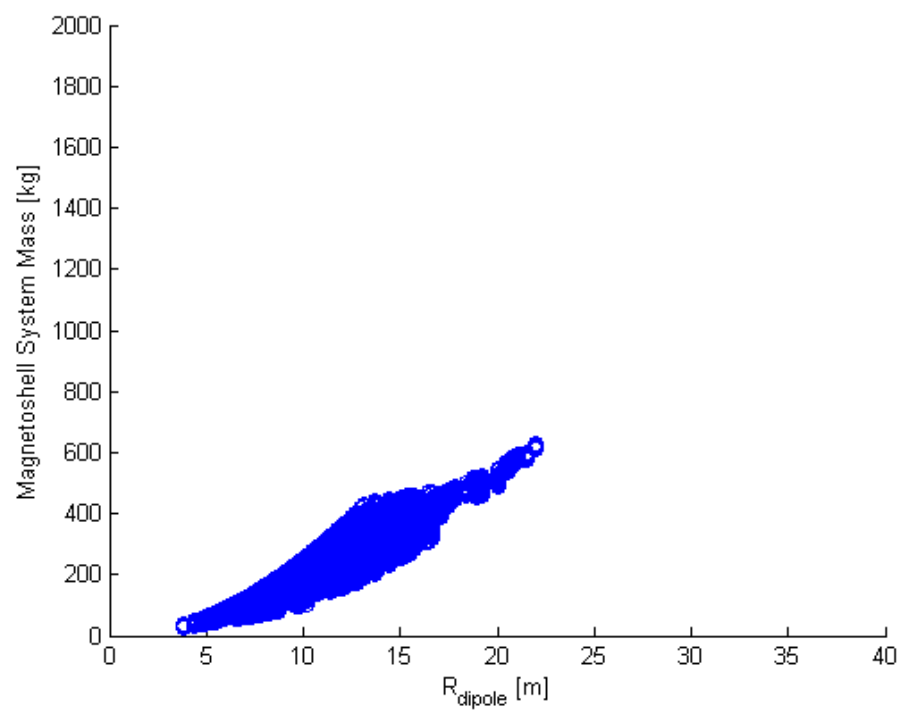

Figure 62. Model solutions for a Mars orbit insertion for a small magnet coil. Shown are all permutations for a wide range of magnet sizes (up to 2.5 meters) and power systems. Battery backed and temperature limited. 
A Plasma Aerocapture and Entry System for Manned Missions and Planetary Deep Space Orbiters

MSNW LLC

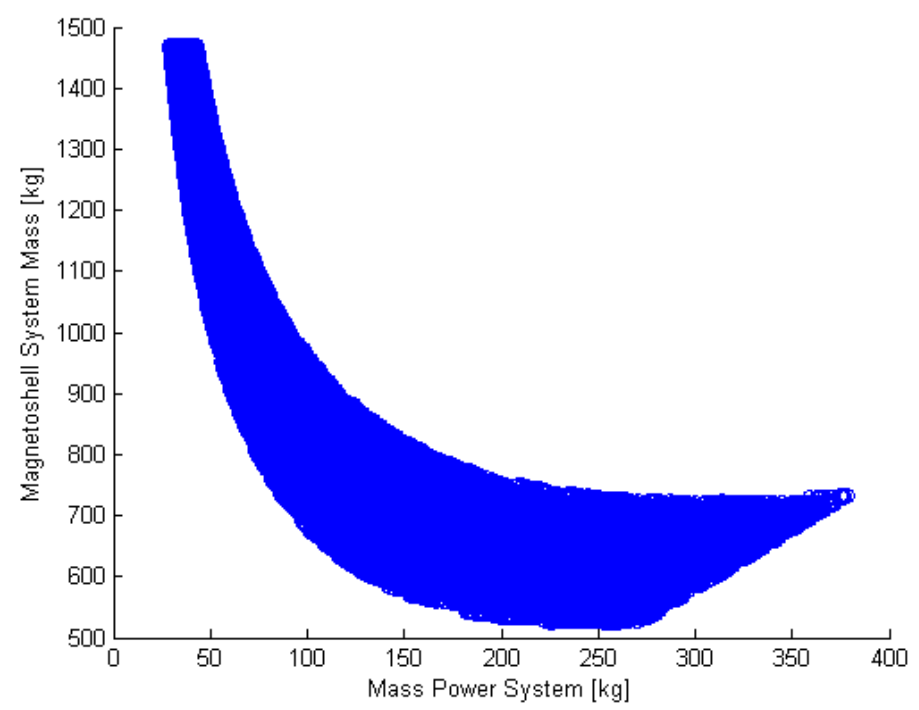

Figure 63. Model solutions for power system (battery, solar) versus total system mass for a Mars orbit insertion. Shown are a fixed $2.5 \mathrm{~m}$ radius magnet.

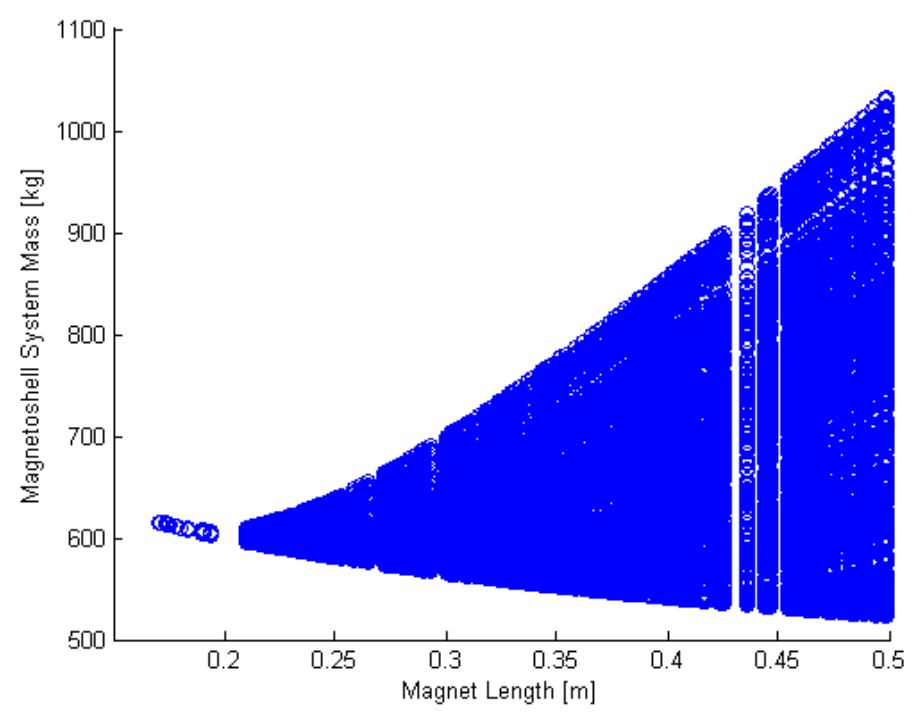

Figure 64. Model solutions for magnet length versus total system mass for a Mars orbit insertion. Shown are a fixed $2.5 \mathrm{~m}$ radius magnet. The wide spread is due to variation in magnet thickness 
A Plasma Aerocapture and Entry System for Manned Missions and Planetary Deep Space Orbiters

MSNW LLC

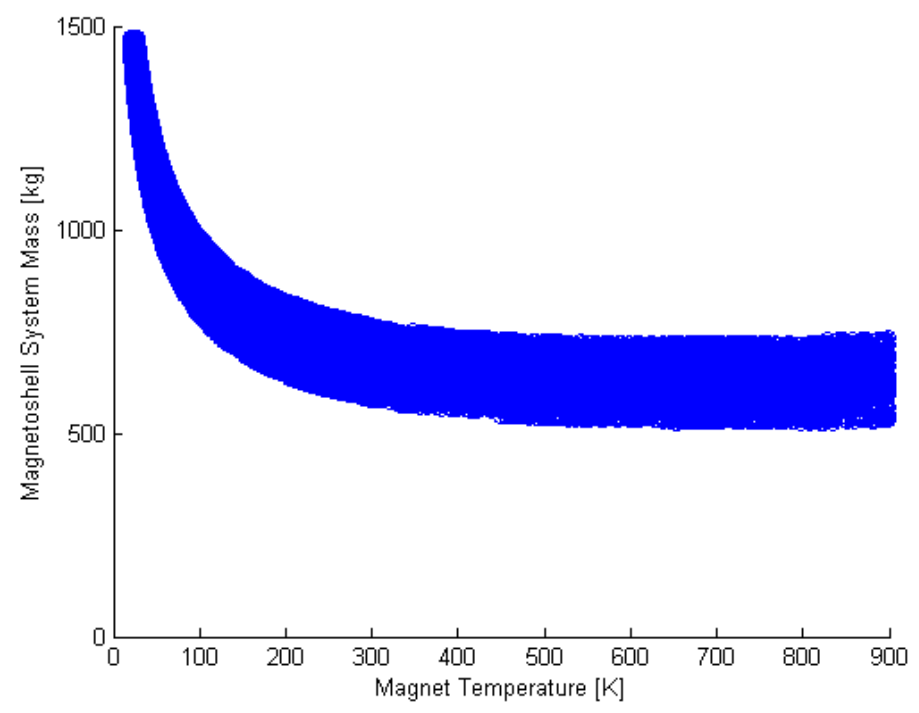

Figure 65. Model solutions for peak operating temperature versus total system mass for a Neptune orbit insertion. Shown are a fixed $2.5 \mathrm{~m}$ radius magnet. Clear minimum system mass for high temperature, but small increases in mass dramatically lower peak temperature.

\begin{tabular}{l|l} 
Martian Magnetoshell Design Parameter & Optimum Configuration \\
\hline Magnet Average Radius & $2.5 \mathrm{Meter}$ Aluminum \\
Total Magnet Weight & $190 \mathrm{~kg}$ \\
Total Battery and Solar Power Weight & $250 \mathrm{~kg}$ \\
Magnet Length & $50 \mathrm{~cm}$ \\
Magnet Thickness & $1 \mathrm{~cm}$ \\
Number of Turns & 8000 \\
Tether Geometry & 30 meters long, $9 \mathrm{~kg}$ \\
Peak Current & 20 Amps \\
Effective Aerobraking Radius & 21 meters \\
Total Weight with 30\% growth & $690 \mathrm{~kg}$ \\
Equivalent Aeroshell Weight & $\sim 20,000 \mathrm{~kg}$
\end{tabular}

Figure 66. Summary of Magnetoshell parameters for a Mars 60 MT insertion. 
A Plasma Aerocapture and Entry System for Manned Missions and Planetary Deep Space Orbiters

MSNW LLC

\section{Mars Orbital Modeling}

Similar to the Neptune study above, a Copernicus simulation of a Martian aerocapture was performed. For this simulation, a 60 metric ton spacecraft is inserted via a simple Hohmann transfer. The atmospheric model used is shown in Figure 67 and comes from the standard Mars atmospheric climate database version 5.0. This is similar to the model used previously; however, is a standard mean; however, is the predicted Martian atmosphere during the arrival date used in Copernicus. Similar to the Neptune study, an initial large Magnetoshell is required to perform the initial insertion and capture. In this simulation, a 21-meter diameter shell is used. The dramatically increased size is required because of the large payload and thus braking energy required to capture into a Martian orbit. An initial capture is performed over the course of five minutes. A then long coast period of 8 days between aerobraking maneuvers. This allows for recalculation of orbital trajectories and adjusting for the dynamic atmosphere. An 18 orbit aerobraking maneuver is then performed to circularize and lower the Mars orbit. Figure 68 through Figure 74 show the orbital results for the Martian braking maneuver with a peak braking force of $0.6 \mathrm{~g}$ 's and dynamic pressure of $130 \mathrm{~N} / \mathrm{m}^{2}$. For reference, the actual calculated ion temperatures for this specific braking maneuver are given.

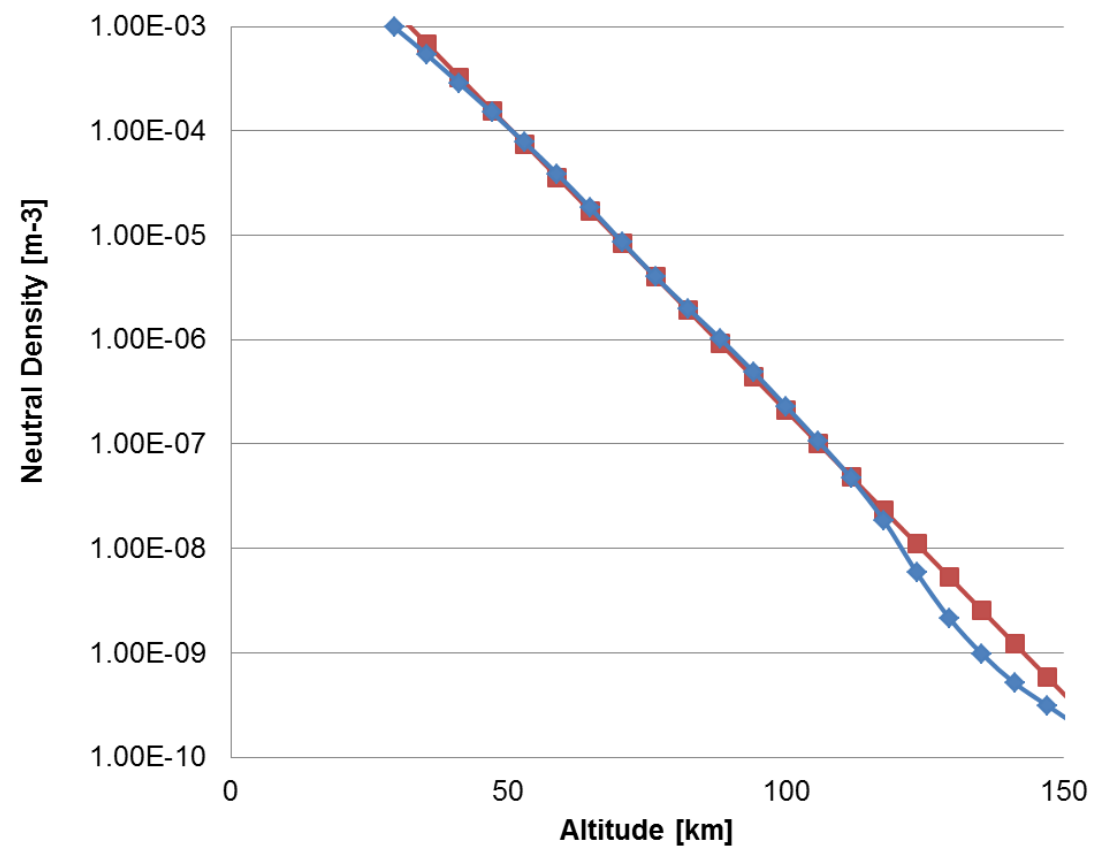

Figure 67. Martian atmospheric model used for COMPERNICUS Aerocapture [15]. Shown in blue is the average atmospheric neutral density, red is the exponential model used. 
A Plasma Aerocapture and Entry System for Manned Missions and Planetary Deep Space Orbiters

MSNW LLC

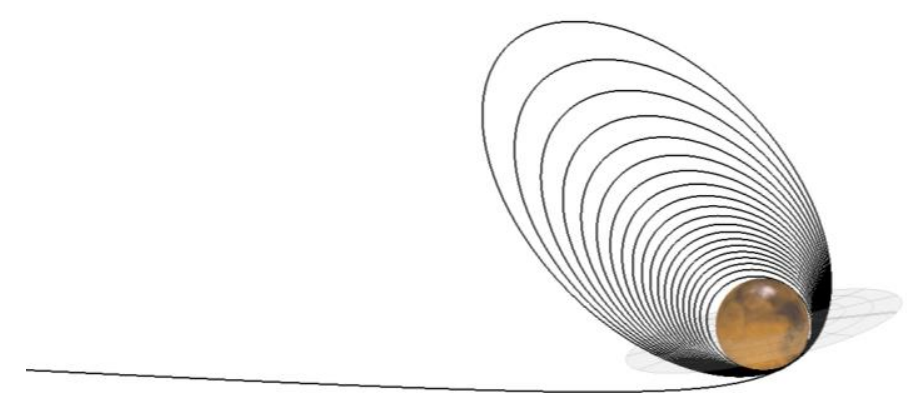

Figure 68. Optimal Mars insertion with mean Martian atmosphere.

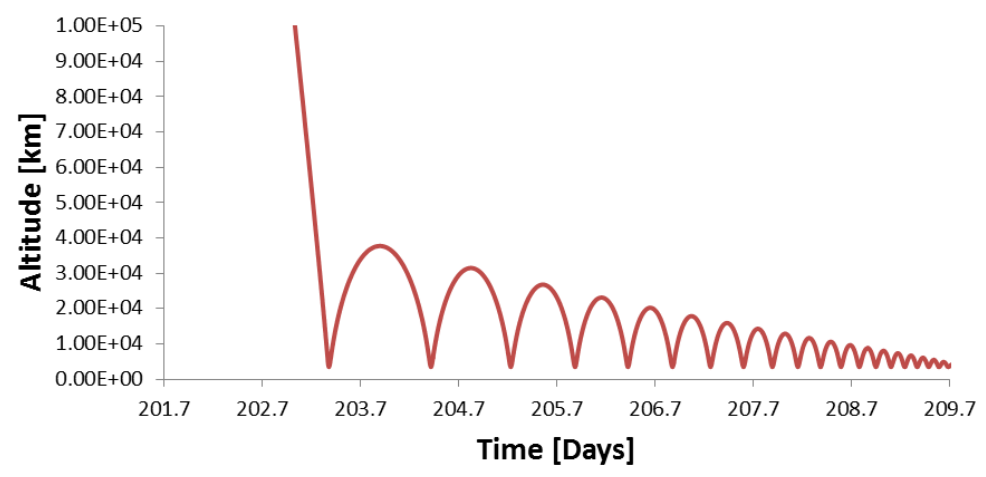

Figure 69. Mars aerocapture altitude.

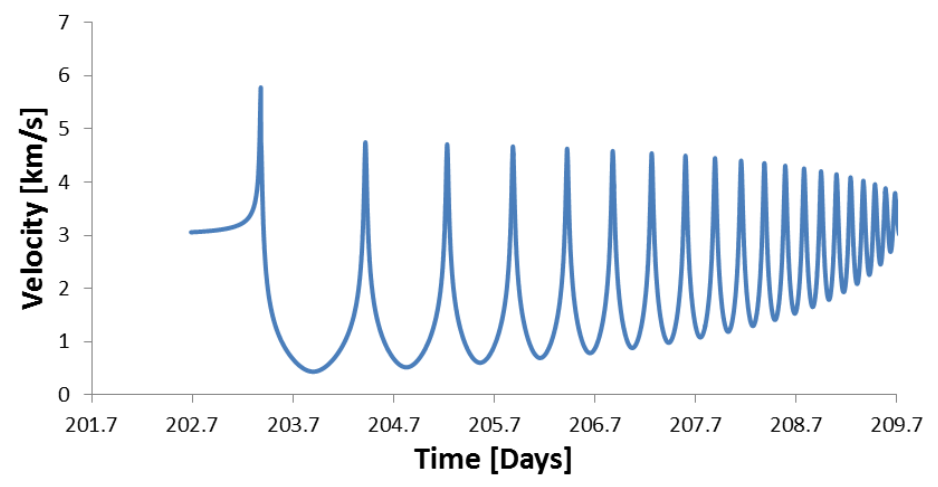

Figure 70. Mars aerocapture velocity. 
A Plasma Aerocapture and Entry System for Manned Missions and Planetary Deep Space Orbiters

MSNW LLC

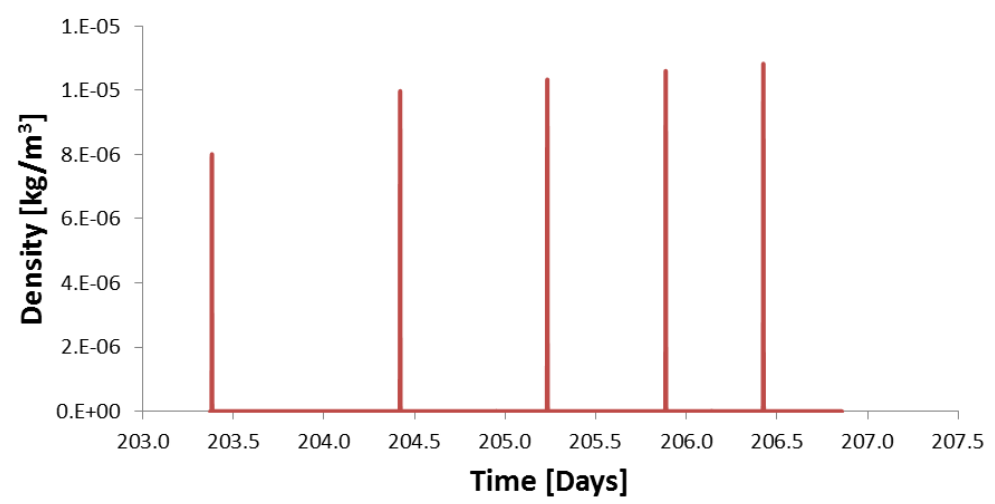

Figure 71. Mars aerocapture atmospheric density.

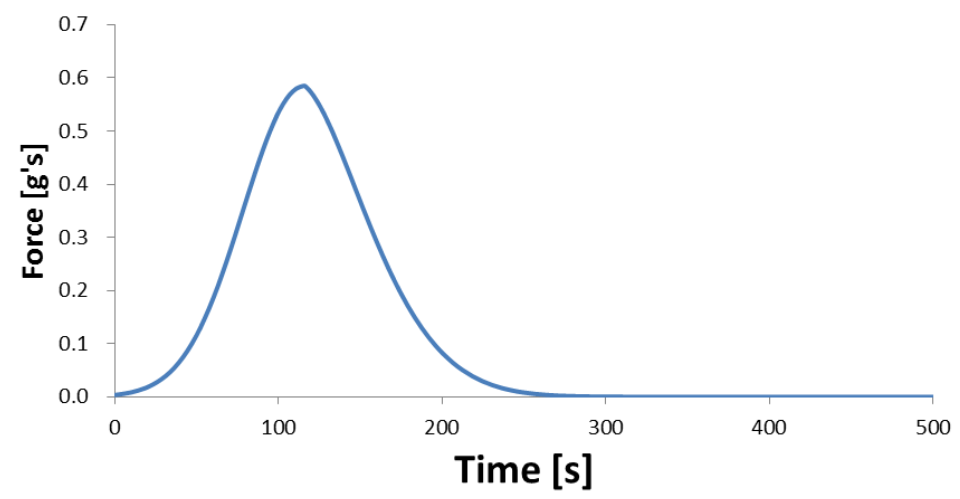

Figure 72. Close up of the Mars drag force for the initial insertion.

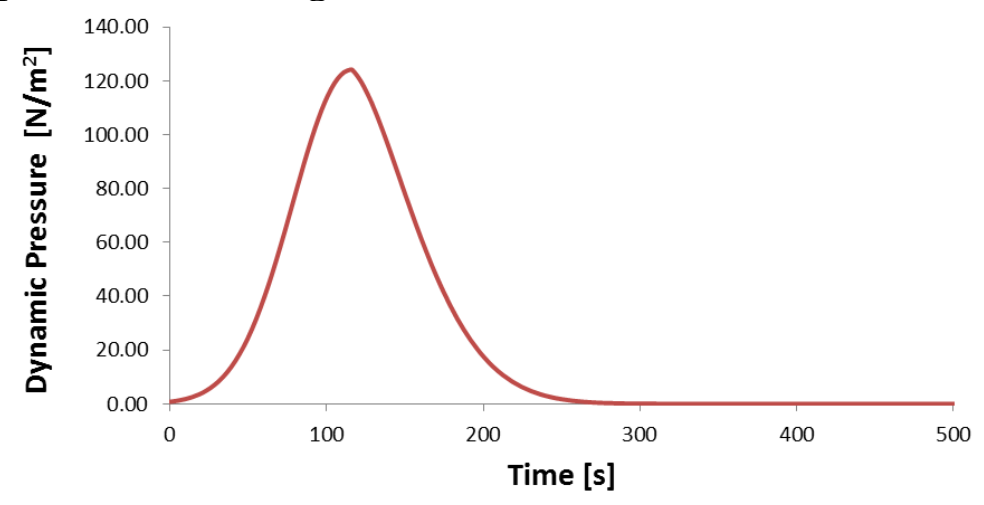

Figure 73. Close up of the Mars dynamic pressure for the initial insertion. 
A Plasma Aerocapture and Entry System for Manned Missions and Planetary Deep Space Orbiters

MSNW LLC

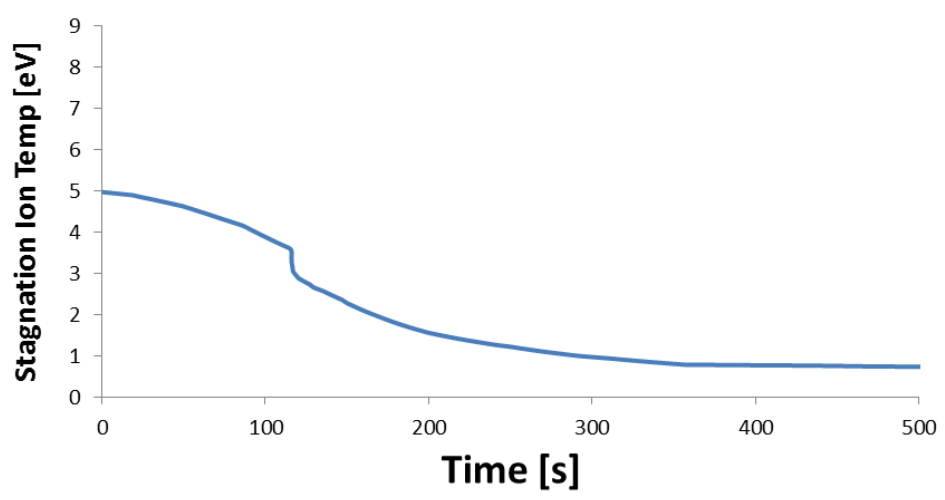

Figure 74. Incoming equivalent ion temperatures.

\section{Risk and Perturbation Analysis}

A risk analysis was undertaken to investigate the ability to dynamically change the shape of the Magnetoshell. Shown in Figure 75 is the optimal drag area for a capture. This area leads to the shedding of orbital velocity, without reentry. As the altitude density decreased exponentially with density it can be seen that small changes in altitude $(10 \mathrm{~km})$ will lead to several orders of magnitude increase in drag force.

\begin{tabular}{c|c|c} 
Apogee Altitude [km] & Optimum Capture Area [ $\left.\mathbf{m}^{\mathbf{2}}\right]$ & Required Aeroshell Radius [m] \\
\hline 70 & $1.4 \mathrm{E} 3$ & 21 \\
80 & $4.8 \mathrm{E} 4$ & 123 \\
90 & $1.2 \mathrm{E} 10$ & $61 \mathrm{~km}$
\end{tabular}

Figure 75. Optimal Aeroshell drag areas for various apogee altitudes.

Further, by simply simulating seasonal atmospheric increase or decrease the resulting trajectories can be simulated. Figure 76 is the optimal insertion trajectory at $70 \mathrm{~km}$ and the mean atmospheric model. By decreasing the density by $1 / 3^{\text {rd }}$ the standard $1400 \mathrm{~m} 2$ shell can no longer capture the $60 \mathrm{MT}$ payload. If the density increases $2 \mathrm{X}$ as in a dust storm or local atmospheric anomaly, the capture trajectory because a full descent trajectory within on orbit, potentially leading to catastrophic burn up (Figure 77). Therefore, the ability to dynamically adjust the drag surface based simply on the drag (and deceleration) of the spacecraft allows the dynamic response of the trajectory (Figure 78) without additional ACS control, propellant, and computing. 
A Plasma Aerocapture and Entry System for Manned Missions and Planetary Deep Space Orbiters

MSNW LLC

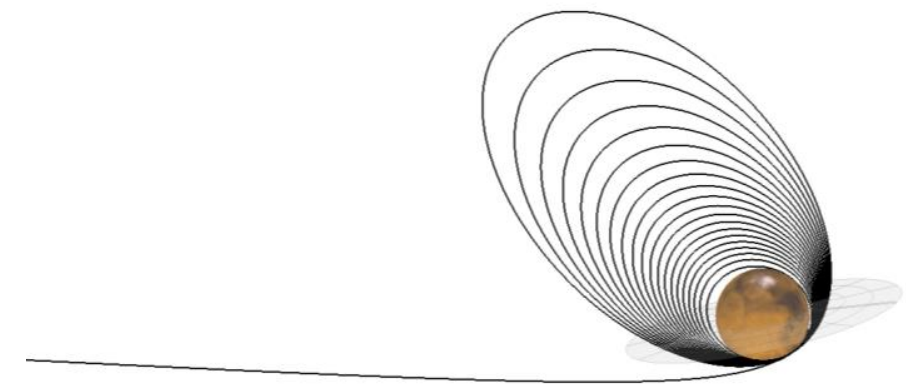

Figure 76. Optimal Mars insertion with mean Martian atmosphere.

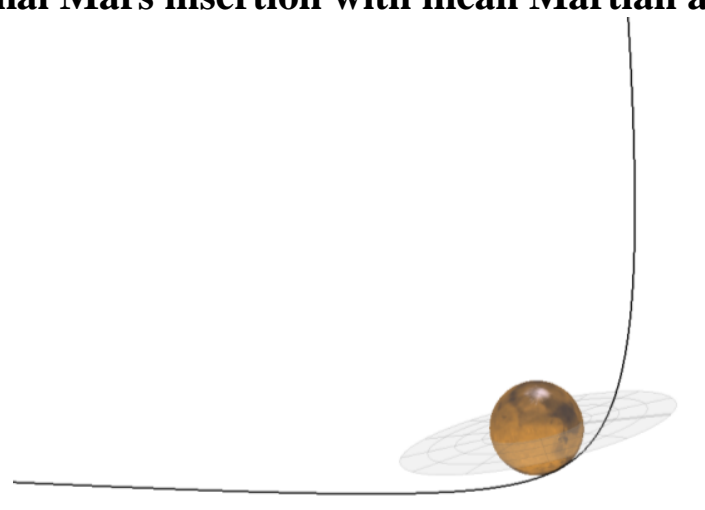

Figure 77. Mars insertion with 3X decrease in atmospheric density leading to hyperbolic escape.

Figure 78. Mars insertion with $2 X$ increase in atmospheric density leading to catastrophic reentry. 
A Plasma Aerocapture and Entry System for Manned Missions and Planetary Deep Space Orbiters

MSNW LLC

\section{Conclusions from the Mission Design Study}

In conclusion, two missions were fully designed and simulated: a Cassini class Neptune orbiter and a HEOMD scale Mars orbital insertion. The complete system design of both missions including Magnetoshell magnet, tether, power processing, and battery mass were scaled and specified. The orbital simulation code Copernicus was used to determine optimal trajectory as well as optimize the magneto shell size as a function of minimal altitude. Some conclusions from these two studies can clearly be drawn. First, Magnetoshell dipole from 2 to 40 meters in radius are easily possible with realistic sized power systems and magnets. For the mission simulated, forces from a few Newtons through many kilo-Newtons were generated allowing for orbital insertion of space craft traveling at greater than $20 \mathrm{~km} / \mathrm{s}$. For a Neptune insertion mission, a 1000 kilogram spacecraft was decelerated and placed into orbit around Neptune using a magneto shell aerocapture; this system with a total of $207 \mathrm{~kg}$, including RTG power requirements. This increased the LEO to Neptune dry payload fraction from $32 \%$ to $40 \%$ and most importantly, allows for the dynamic capture behavior as a function of the real-time Neptune atmosphere.

For Mars insertion, the primary benefit is mass savings. Any 20 MT aero shell could be replaced with a Magnetoshell system of less than one metric ton. This saves over 20 MT of launched propellant per launch. The Martian insertion was capable of supporting a 60 metric ton payload and fitting into a standard faring size. This mission is not possible without an aerocapture system and the authors believe that a Magnetoshell system both decreases cost as well as risk. It is thereby likely that once thoroughly demonstrated, a Magnetoshell system can be used for aerocapture on manned, on crewed missions, further decreasing propellant requirement.

\begin{tabular}{l|l} 
Neptune Magnetoshell Design Parameter & Optimum Configuration \\
\hline Magnet Average Radius & 2 Meter Aluminum \\
Total Magnet Weight & $81 \mathrm{~kg}$ \\
Total Battery and Solar Power Weight & $72 \mathrm{~kg}$ \\
Magnet Length & $3.5 \mathrm{~cm}$ \\
Magnet Thickness & $3.5 \mathrm{~cm}$ \\
Effective Aerobraking Radius & $9 \mathrm{Meters}$ \\
Total Weight with 30\% growth & $207 \mathrm{~kg}$ \\
Equivalent Aeroshell Weight & $885 \mathrm{~kg}$
\end{tabular}

\begin{tabular}{l|l} 
Martian Magnetoshell Design Parameter & Optimum Configuration \\
\hline Magnet Average Radius & 2.5 Meter Aluminum \\
Total Magnet Weight & $190 \mathrm{~kg}$ \\
Total Battery and Solar Power Weight & $250 \mathrm{~kg}$ \\
Magnet Length & $50 \mathrm{~cm}$ \\
Magnet Thickness & $1 \mathrm{~cm}$ \\
Effective Aerobraking Radius & 21 meters \\
Total Weight with 30\% growth & $690 \mathrm{~kg}$ \\
Equivalent Aeroshell Weight & $\sim 20,000 \mathrm{~kg}$
\end{tabular}


A Plasma Aerocapture and Entry System for Manned Missions and Planetary Deep Space Orbiters

MSNW LLC

\section{Overall Phase I NIAC Conclusions}

A plasma Magnetoshell can enable a wealth of large scale inner planetary missions and deep space planetary orbiters. The Magnetoshell aerocapture deploys a magnetic field filled with a magnetized plasma. It is interaction of the atmosphere with this plasma that supplies a significant impediment to atmospheric flow past the spacecraft, and thereby producing the desired drag for braking.

In Phase I a full system was designed for Neptune and Mars missions. This analysis showed that a $200 \mathrm{~kg}, 9 \mathrm{~m}$ Magnetoshell provides Neptune aerocapture for a $21 \mathrm{~km} / \mathrm{s}$ injection with a peak force of $150 \mathrm{~N}$. For a manned Martian aerocapture, a 21 meter Magnetoshell can be developed to provide aerocapture for a 60 metric ton payload. A transient analytic model was developed evolving the radial plasma parameters for a variety of plasma, neutral, and magnetic parameters. Finally, a stationary 2 meter argon Magnetoshell was fully demonstrated and a 1000:1 increase in aerodynamic drag was found.

\section{References}

[1] John Slough, "Plasma Sail Propulsion Based on the Plasma Magnet", IEPC-2007-15, 30th International Electric Propulsion Conference, Florence, Italy September 17-20, 2007.

[2] Krasheninnokov, S.I., Catto, J.J., Hazeltine, R.D., "Magnetic Dipole Equilibrium Solution at Finite Plasma Pressure", Physical Review Letters, 82 (1999) p2689.

[3] Winglee, R.M., Slough, J.T., Ziemba, T., and Goodson, A., "Mini-magnetospheric plasma propulsion: High speed propulsion sailing the solar wind", Space Technology and Applications (2000).

[4] Zubrin, R.M. and Andrews, D.G., "Magnetic Sails and Interplanetary Travel", Journal of Spacecraft, 28197 (1991).

[5] Atmosphere, US Standard. "National Oceanic and Atmospheric Administration." National Aeronautics and Space Administration, and United States Air Force, Washington, DC (1976).

[6] Peter A, Gnoffo, et al. "Prediction and Validation of Mars Pathfinder Hypersonic Aerodynamic Data Base." (1998).

[7] Justus, C. G., Aleta Duvall, and Vernon W. Keller. "Atmospheric Models for Aerocapture Systems Studies." AIAA Paper 4952 (2004).

[8] Lotz, Wolfgang. "Electron-impact ionization cross-sections and ionization rate coefficients for atoms and ions." The Astrophysical Journal Supplement Series 14 (1967): 207.

[9] Losev, S. A., et al. Physical and chemical processes and gas dynamics: cross sections and rate constants. Progress in Astronautics and Aeronautics 196 (2002).

[10] Smirnov, Boris M. Physics of atoms and ions. Springer, 2003.

[11] Meier, E.T., et al. "Development and validation of a two-fluid plasma-neutral model", Innovative Confinement Concepts (2011).

[12] A.H. Glasser, X.Z. Tang, Comput. Phys. Commun. 164 (2004) 237

[13] E.T. Meier, U. Shumlak , R.D. Milroy, D. Kirtley, J. Slough, "Development and validation of a two-fluid plama-neutral model”, Innovative Confinement Concepts Workshop, 2011. 
A Plasma Aerocapture and Entry System for Manned Missions and Planetary Deep Space Orbiters

MSNW LLC

[14] Kirtley, D., Slough, J., Pihl, C. "Pulsed Plasmoid Propulsion: Air-Breathing Electromagnetic Propulsion”. International Electric Propulsion, Conference, 2011.

[15] Kirtley, D.E., Slough, J.T., and Votroubek, G. "Neutral Entrainment Demonstration in a Xenon FRC Thruster Experiment”, Joint Army Navy NASA Air Force Conference, 2013

Kawaguchi, J.; Ichikawa, T.; Nishimura, T.; Uesugi, K.; Efron, L.; Ellis, J.; Menon, P. R.; Tucker, B., "Navigation for Muses-A (HITEN) aerobraking in the earth's atmosphere Preliminary report",Institute of Navigation, Annual Meeting, 47th, Williamsburg, VA, June, 1991, p. 17-27.

Giorgini, J., Wong, S. K., You, T.-H., Chadbourne, P., Lim, L., "Magellan Aerobrake Navigation”, British Interplanetary Society, Journal, vol. 48, no. 3, p. 111.

[18] Daniel T. Lyons, Joseph G. Beerer, Pasquale Esposito, and M. Daniel Johnston, "Mars Global Surveyor: Aerobraking Mission Overview", Journal of Spacecraft and Rockets. 36 (1999) p307.

[19] Vaughan, D., Miller, H. C., Griffin, B., James, B.F., and Munk, M. M., "A Comparative Study of Aerocapture Missions with a Mars Destination", 41st AIAA/ASME/SAE/ASEE Joint Propulsion Conference and Exhibit, Tucson, Ariz, USA, July, 2005.

[20] J. Slough, "Plasma Magnetic Shield for Crew Exploration", NIAC Phase I Final Report, 2007.

[21] Munk, M., and Spilker, T. "Aerocapture Mission Concepts for Venus, Titan and Neptune" International Planetary Probe

[22] Spilker, Thomas R. "A Non-NEP Implementation of NASA's "Neptune Orbiter With Probes" Vision Mission." AIP Conference Proceedings. Vol. 746. 2005.

Workshop (2008).

[23] Ritchie, A. G. "Recent developments and future prospects for lithium rechargeable batteries." Journal of power Sources 96.1 (2001): 1-4.

[24] "Human Exploration of Mars, Design Reference Architecture 5.0" July 2009, NASA-SP2009-566.

[25] Mars atmospheric database Mars Climate Database (c) LMD/OU/IAA/ESA/CNES. 